\title{
Prices and volumes of options: A simple theory of risk sharing when markets are incomplete*
}

\author{
Francois Le Grand and Xavier Ragot ${ }^{\dagger}$
}

February 8, 2010

\begin{abstract}
We present a simple theory of business cycle movements of derivative asset prices and volumes. This theory relies on time-varying heterogeneity among agents in their demand for insurance against aggregate risk. Formally, we build an infinite horizon model in which agents face an aggregate risk, but also different levels of idiosyncratic risk. We are able to analytically characterize a general equilibrium in which positive quantities of derivatives are traded. This allows us to explain the informational content of derivative volumes over the business cycle. We reproduce stylized facts about derivative volumes, and also perform welfare analysis with respect to the introduction of derivative assets, which notably appears to be not Pareto improving.
\end{abstract}

Keywords: Option Pricing, Open Interest, Incomplete Markets

JEL codes: G10, G12, E44

\section{Introduction}

Derivative assets are financial instruments allowing agents to exchange risks. The volume of exchanged derivatives contains information about the opportunities of risk trading, and thus about the heterogeneity among agents in their risk assessment. This volume is time-varying and depends on the volatility of the underlying asset price. Considering one of the most traded derivatives, which are options on the S\&P 500 index, lets notably appear that the traded volume increases with the volatility of the underlying asset, the S\&P 500. More aggregate risk thus generates more

\footnotetext{
*Acknowledgments: We are indebted to Gabrielle Demange and Guy Laroque for helpful suggestions. We also thank participants at the joint HEC-INSEAD-PSE Workshop 2009, the 2009 American and Far-Eastern Meetings of the Econometric Society, Seminars in Crest, Paris School of Economics, Paris-Dauphine University, and EMLyon Business School for valuable comments.

†legrand@em-lyon.com and xavier.ragot@banque-france.fr
} 
heterogeneity and modifies the willingness to bear risks. We develop a general equilibrium model with incomplete markets, where this time varying heterogeneity is endogenously derived and which is consistent with the previous basic fact. We provide general pricing equations when options are not redundant and use the model to analyze the welfare effect of introducing a derivative asset.

The model we present here departs from the seminal Black and Scholes (1973) setting, in which options are redundant and priced by replication. In this setup, option volumes are not determined and the exchange of insurance among agents does not play any specific role. We study a deviation from the standard infinite horizon economy à la Lucas, endowed with a single exogenous risky asset, where agents face heterogeneous uninsurable idiosyncratic risks and borrowing constraints. As a consequence of this last assumption, agents differ in their willingness to bear the aggregate risk on the top of the heterogeneous idiosyncratic risk. Incomplete markets for some risk thus provide a foundation for the aggregate risk sharing and the exchange of insurance thanks to derivative assets. This motivation is appealing, since the assumption of market incompleteness receives empirical support and has been introduced to contribute to the solution of many pricing puzzles, like the equity premium (Constantinides and Duffie (1996)) and the risk free rate (Huggett (1993)). In this paper, we derive the implication of this assumption on the pricing of derivatives, on their volume determination and on the welfare effect of their introduction.

This foundation of the demand for derivative assets has been studied in the literature before, notably by Franke, Stapelton and Subrahmanyam (1998), in a two-date economy. Our contribution consists in introducing a tractable infinite horizon setting where pricing formula can be easily derived and where welfare analysis is possible. We are able to do so because we generalize the work of Challe, Le Grand and Ragot (2007), who present an analytical framework with both aggregate and insurable idiosyncratic risks. In this equilibrium, the ex post heterogeneity is reduced to a finite number of different agents. We thus avoid the computation of an approximate equilibrium, which is possible only with a reduced number of assets (typically two) in the presence of aggregate shocks. On the contrary, our setup allows for the introduction of an arbitrary high number of derivatives. More specifically, we model the idiosyncratic risk as an employment risk and makes two assumptions to reduce the heterogeneity. The first one is that the labor supply is so highly elastic (as in Scheinkman and Weiss (1986) and in Lagos and Wright (2005)), that employed compensate income fluctuations by working more. The second assumption is that the income when unemployed (unemployment benefit or home production) is such that unemployed agents choose to liquidate their complete portfolio when falling into unemployment and choose to hit the credit constraint. In this setup, we introduce heterogeneity across agents by allowing for different idiosyncratic risks and thus for various types of agents. Although this framework is highly stylized, it allows us to clearly identify the equilibrium exchange of risk under incomplete market and in a 
tractable model. The gain is to be able to provide new results concerning the interaction between option trading and time-varying aggregate risk.

The first part of the paper features two types of agents with two different levels of idiosyncratic risks, who buy shares of a risky tree, both to smooth consumption and to self-insure against uninsurable income risk. We study this economy with and without a single call option, which completes the market in this simple framework. We analyze how the availability of the derivative asset affects the portfolio structure of both agents. The agents facing the lowest level of idiosyncratic risk choose to take a larger share of aggregate risk by purchasing call options. Since the call pays off only in the good state of the world, the low risk agent chooses when purchasing call options, to hold a riskier portfolio and thus to bear a larger share of the aggregate risk and to provide insurance to the high risk agent, who has sold the option.

We prove that the volume of traded options increases when the heterogeneity and the exposure to idiosyncratic risk increases and when the dividends of the risky asset become more volatile. This model is thus able to reproduce the correlation between the asset volatility and the volume of options reported in the data. The intuition for these results is the following. An increase in the riskiness of the asset, which can be thought as a mean-preserving spread of dividends, implies that in some states of the world the payoff of the risky asset is low. Agents want to insure themselves against the conjunction of a bad idiosyncratic outcome and a bad aggregate shock, which means a low portfolio liquidation value, while being unemployed. As the idiosyncratic risk differs across agents, the agents with a low idiosyncratic risk will be willing to bear a larger share of the aggregate risk than agents with the high level of idiosyncratic risk. High risk agents purchase a larger quantity of underlying asset and also sell call options (i.e. buy insurance) in order to smooth their portfolio payoffs across states of the world.

Second, we show that the introduction of the option increases the welfare of the agents facing the high level of idiosyncratic risk and decreases the welfare of agents facing the low level of idiosyncratic risk. The first ones are more able to self-insure themselves and bear a smaller share of the aggregate risk after the sale of options. The other agents' type purchases options and holds a riskier portfolio. They support a larger share of the aggregate risk and their welfare decreases. The option introduction is therefore not Pareto improving, which illustrates Elul (1995) and Cass and Citanna (1998) papers about the impact of financial innovation on welfare.

The second version of the model generalizes the previous setup. An arbitrarily high number of different agents' types facing different level of idiosyncratic risks are able to trade an arbitrarily large number of options derived from a single risky Lucas tree. The technical part consists in proving the equilibrium existence and in deriving the joint equations for portfolio compositions and prices. As an empirical illustration, we calibrate the model to reproduce roughly volumes and 
prices of options in a simplified representation of business cycles.

The rest of the paper is organized as follows. The next section is the literature review. The third section presents the simple economy with only one risky asset. Section 4 introduces options to complete the market for the aggregate risk. The final section generalizes the equilibrium existence to an economy with an arbitrarily large number of agents and of securities.

\section{Literature review}

Our paper belongs first to the option pricing literature allowing for heterogeneity and deriving simultaneously option prices and quantities. Three reasons justifying the aggregate risk sharing among agents can be found in the literature. The first one is that agents have different preferences and notably different risk aversion. As a consequence, they value differently the various aggregate risks (Bhamra and Uppal (2009)). The second one is that agents have different beliefs or information about the aggregate risk (Biais and Hillion (1994); Buraschi and Jiltsov (2006)). The third one is that agents face different background risks. Their willingness to bear aggregate risk on the top of this idiosyncratic (or background) risk differs (Franke, Stapelton and Subrahmanyam, (1998)). Our model is related to this third type of motivation. Our contribution is to depart from a two-date model and to propose an infinite horizon economy, in which we characterize analytically prices and volumes and perform a welfare analysis.

There is a large number of papers in the microstructrure literature documenting the stylized fact that we reproduce, which is the positive relationship between the underlying volatility and the activity on the option market. Easley, O'Hara and Srivinas (1998) exhibit a positive correlation between contemporaneous stock price changes and option volumes. Donders, Kouwenberg and Vorst (2000) find the same positive relationship using earning announcements. Moreover, a survey of Karpoff (1987) reports a strong positive link between stock price changes and the trading volume in the stock market. In addition, Anthony (1988) and Stephan and Waley (1990) find a positive correlation between stock volume and option volumes. These both results together confirm the relationship between stock prices and option volumes. An empirical result closer to our objectives and our model is the positive correlation between the option open interest and volatility of the S\&P 500 that Buraschi and Jiltsov (2006) find using daily data on the S\&P 500 index. There is a strong consensus about the positive relationship, but there is a debate about the informational content of the derivative volume for predicting future stock prices (Easley, O'Hara and Srivinas (1998) or Pan and Poteshman (2006) among others) or future stock volatility (Ni, Pan and Poteshman (2008)), often on very short horizons (indraday or less than a week). Obviously, this aspect is far beyond the scope of our paper and we are only interested in option volumes over 
the business cycles.

Our paper is also related to no-trade equilibria models, which allow for analytical prices in an infinite horizon economy with incomplete markets. Constantidines and Duffie (1996) propose such a framework to solve the equity premium. Krusell, Mukoyama, and Smith (2008) investigate asset prices in a no-trade equilibrium, in order to derive simultaneously bond and equity prices. Our paper is also a generalization of our previous work (Challe, Le Grand and Ragot (2007)), where we studied the term structure of interest rates in the vicinity of the no-trade equilibrium, but with positive trades of bonds. We add here the ex-ante heterogeneity in the idiosyncratic risk agents face, allowing us to study the risk sharing over the business cycle through the option trading.

\section{A simple economy with one risky asset}

We consider an economy with one risky asset, where two types of agents (type 1 and type 2) face an uninsurable unemployment shock. The risky asset is a simple Lucas tree, whose mass $V$ remains constant through time. The tree pays off at each date $t$ a dividend $y_{t}$ in consumption goods, whose variations are the single aggregate risk in the economy. These dividends can only take two values in $Y=\left\{y_{G}, y_{B}\right\}$, with $y_{G} \geq y_{B}: G$ refers to the good state, while $B$ to the bad state. Dividends evolve as a two state Markov chain, whose transition probabilities to move from state $k$ to state $l$ $(k, l=G, B)$ are denoted $\pi_{k l}$. To avoid the discussion of uninteresting cases, we make the following assumption.

Assumption A (Persistence) Both aggregate states are persistent, i.e. $\pi_{G G}+\pi_{B B}>1$.

The previous assumption states that aggregate states are persistent and do not fluctuate too often, what is consistent with data 1 The price of one unit of the tree at date $t$ is denoted $P_{t}$.

In addition to this aggregate risk, all agents face an uninsurable unemployment risk: Agents can be either employed or unemployed. When employed, type 1 agents face a probability $\alpha^{1}$ to become unemployed an thus a probability $1-\alpha^{1}$ to stay employed. When unemployed, these type 1 agents face a probability $\rho^{1}$ to stay unemployed and a probability $1-\rho^{1}$ to find a job. With obvious notations, type 2 agents face the same risk with probabilities $\alpha^{2}$ and $\rho^{2}$. Transition probabilities to get into and out of unemployment are constant, so that we can deduce the long-run fraction $\eta^{i}$ of type $i$ employed agents:

$$
\text { for } i=1,2 \quad \eta^{i}=\frac{1-\rho^{i}}{1+\alpha^{i}-\rho^{i}}
$$

The initial fraction of employed agents of type $i$ is also assumed to be $\eta^{i}$ to avoid transitory dynamics.

\footnotetext{
${ }^{1}$ For instance, Hamilton ((1994), chap 22) finds $\pi_{G G}+\pi_{B B}=1.65$ at a quarterly frequency for the US economy.
} 
When employed, agents earn a constant hourly wage that we normalize to 1 and they can freely adjust their labor supply. When unemployed, agents benefit from a constant "domestic production" that we note $\delta>0$. Home production is low enough for unemployed agents to be worse-off compared to employed ones, which insures that agents are willing to participate to the labor market. More formally, we make the following assumption.

Assumption B (Labor market participation) $u^{\prime}(\delta)>1$.

\subsection{Preferences and the agents' program}

In each period, agents enjoy utility from consumption and leisure. Preferences are separable in time, as well as in consumption and leisure. More precisely the periodic utility of any agent over consumption $c$ and labor $l$ expresses as $u(c)-l$, where $u: \mathbb{R} \rightarrow \mathbb{R}$ is twice derivable, increasing and concave. We follow indeed Scheinkman and Weiss (1986) and Lagos and Wright (2005) among others and assume that agents are infinitely elastic in labor. Instantaneous preferences are discounted with a common factor $\beta \in(0,1)$, representing the time decay. We discuss later the implications of the linear disutility of labor.

We denote $e_{t}^{i} \in\{0,1\}$ the employment status of a type $i$ agent $(i=1$ or 2$)$, with $e_{t}^{i}=1$ when he is employed and $e_{t}^{i}=0$ otherwise. The program of a type $i$ agent consists in maximizing his intertemporal utility under a set of constraints, by choosing his consumption level, his labor supply and his asset demand, which are noted respectively $c_{t}^{i}, l_{t}^{i}$ and $x_{t}^{i}$. The operator $E_{0}[\cdot]$ is the unconditional expectation over aggregate and idiosyncratic shocks.

$$
\begin{array}{ll}
\max _{c^{i}, l^{i}, x^{i}} & E_{0} \sum_{t=0}^{\infty} \beta^{t}\left(u\left(c_{t}^{i}\right)-l_{t}^{i}\right) \\
\text { s.t. } & c_{t}^{i}+P_{t} x_{t}^{i}=e_{t}^{i} l_{t}^{i}+\left(1-e_{t}^{i}\right) \delta+\left(P_{t}+y_{t}\right) x_{t-1}^{i} \\
& c_{t}^{i}, l_{t}^{i} \geq 0 \text { and } x_{t}^{i} \geq 0 \\
& \lim _{t \rightarrow \infty} \beta^{t} u^{\prime}\left(c_{t}^{i}\right) x_{t}^{i}=0
\end{array}
$$

The initial asset endowment is noted $x_{-1}^{i}$. The agent of type $i=1,2$ maximizes his expected intertemporal utility (1) subject to a set of constraints (2)-(4). According to the constraint (2), total resources made of labor income (or domestic production for unemployed), asset dividends and asset sale values are used to consume and to purchase assets. The second condition (3) states that the consumption level and the labor supply have to remain positive, which will always be the case in all equilibria that we consider. The borrowing constraint stating that agents are prevented from short selling the tree appears in equation (3). The last equation (4) simply features the transversality condition that will hold in our equilibria. 
The agents' risk sharing is limited along three dimensions, since agents are only allowed to trade positive quantities of a single asset. First, the market for the aggregate risk is incomplete, because there is a single asset, while the aggregate risk takes two possible values. Second, the unemployment individual risk is uninsurable, because there is no asset contingent to labor status. Finally, agents can only trade positive asset amounts, which limit their portfolio compositions. This set of restrictions will be relaxed in the next section, where we complete the market for the aggregate risk. These assumptions about the idiosyncratic risk is common to the literature on liquidity constraints, called the heterogeneous agents literature (see for instance Krusell, Mukoyama, and Smith (2008)).

We define $\phi_{t}^{i} \geq 0$ as the Lagrange multiplier associated to the borrowing constraint (3). Writing the Lagrangian of the previous program allows us to derive the following first order conditions:

$$
\begin{gathered}
\text { labor market condition: }\left\{\begin{array}{lll}
u^{\prime}\left(c_{t}^{i}\right)=1 & \text { if } \quad e_{t}^{i}=1 \\
l_{t}^{i}=0 & \text { if } \quad e_{t}^{i}=0
\end{array}\right. \\
\text { consumption smoothing: } P_{t} u^{\prime}\left(c_{t}^{i}\right)=\beta E_{t}\left[u^{\prime}\left(c_{t+1}\right)\left(P_{t+1}+y_{t+1}\right)\right]+\phi_{t}^{i}
\end{gathered}
$$

The first order condition (5) expresses that while an unemployed agent does not supply any labor, an employed agent works, such as to equalize his consumption marginal utility to the constant marginal pain of labor, equal to 1 . In consequence, all employed agents chose the same consumption level. The Euler equation (6) equalizes the cost of giving up today one unit of consumption to the expected $\left(E_{t}[\cdot]\right.$ is the expectation over both aggregate and idiosyncratic states, conditional on the whole available information at date $t$ ) gain of one additional unit of consumption tomorrow. When the Lagrange multiplier $\phi_{t}^{i}$ is strictly positive, the type $i$ agent would like to borrow and to consume more, but his binding credit constraint prevents him to do so.

\subsection{Market clearing and equilibrium}

The asset market clearing condition simply equalizes the asset supply, which is constant and equal to $V$, to the overall asset demand. To aggregate individual demands, we define the probability measure $\Lambda_{t}^{i}: \mathbb{R} \times E \rightarrow \mathbb{R}$ describing the distribution of type $i$ agents as a function of their asset holdings and labor status. This probability is consistent with the history of both aggregate and idiosyncratic shocks. As an example, $\Lambda_{t}^{2}(x, 1)$ is the measure of employed agents of type 2 holding an asset quantity $x$. The market clearing condition expresses therefore as follows:

$$
\int_{\left(x^{1}, e\right) \in \mathbb{R}^{+} \times E} x^{1} d \Lambda_{t}^{1}\left(x^{1}, e\right)+\int_{\left(x^{2}, e\right) \in \mathbb{R}^{+} \times E} x^{2} d \Lambda_{t}^{2}\left(x^{2}, e\right)=V
$$

The Walras law implies that the good market clears as soon as the asset market does. In consequence, we are able to define an equilibrium in our economy as follows: 
Definition 1 (Equilibrium) For an initial distribution of asset holdings $\left(x_{-1}^{i}\right)_{i=1,2}$ and employment status $\left(\Lambda_{0}^{i}\right)_{i=1,2}$ for both agents' types, an equilibrium consists in individual choices $\left\{c_{t}^{i}, l_{t}^{i}, x_{t}^{i}\right\}_{t=0, \ldots, \infty}^{i=1,2}$ and a price sequence $\left(P_{t}\right)_{t=0, \ldots, \infty}$, such that:

1. Given prices, individual strategies solve the agents' optimization program (1).

2. The distribution of $\Lambda_{t}^{i}(i=1,2$ and $t \geq 0)$ is consistent with both aggregate and idiosyncratic state evolutions for both agents' types.

3. The asset market clears (i.e. the equality (7) holds).

\subsection{Reduced heterogeneity equilibrium}

\subsubsection{Assumptions and description}

In standard economies featuring uninsurable idiosyncratic shocks and credit constraints, the equilibrium cannot be explicitly derived, since it implies an infinite distribution of agents' with different individual histories. The usual strategy consists in computing approximate equilibria. In this paper, we generalize a previous work (Challe, Le Grand and Ragot (2007)), to derive an equilibrium where the heterogeneity in the demand for insurance can be computed with paper and pencils.

This equilibrium is based on two assumptions. The first one has already been introduced and is the linearity in the disutility of labor. In this case, when employed, agents are able to freely adjust their labor supply to obtain a constant marginal utility for consumption, equal to 1 (equation (5)). All employed agents consume thus the same amount. This assumption is introduced to decrease the heterogeneity across employed agents.

Our second assumption is that the quantity $V$ of the asset remains small enough, such that even after selling their whole portfolio, unemployed agents are credit constrained. In other words, we assume that the asset quantity is not sufficient for agents to overcome their credit constraint after falling into unemployment. More formally, this assumption states that the Lagrangian multiplier of the credit constraint (6) in the agent's program binds if and only the agent is unemployed - and is slack when he is employed, as summarized in the following assumption:

Assumption C (Small asset volumes) Unemployed agents are always credit constrained, while employed ones always participate to the asset market:

$$
\forall i \in\{1,2\}, \phi_{t}^{i}>0 \Leftrightarrow e_{t}^{i}=0
$$

Of course, this assumption as to be checked in equilibrium. In Section 3.3 .2 below, we show that this condition will be fulfilled when $V$ is small enough. A second implication of this assumption is 
that all employed agents, whatever their type, purchase the asset. This is insured by the assumption that the idiosyncratic risks of the two types of agents are not too different, or that $\alpha^{1}$ is not very different from $\alpha^{2}$.

In this equilibrium, it is easy to write the Euler equation (6) of employed agents of type $i$, separating aggregate from idiosyncratic states, which expresses as:

$$
P_{t}=\beta\left(1-\alpha^{i}\right) E_{t}\left[P_{t+1}+y_{t+1}\right]+\beta \alpha^{i} E_{t}\left[u^{\prime}\left(\delta+\left(P_{t+1}+y_{t+1}\right) x_{t}^{i}\right)\left(P_{t+1}+y_{t+1}\right)\right]
$$

The left hand side is the opportunity cost to buy one unit of asset at period $t$. The first term at the right hand side is the valuation of expected return from holding the asset if the agent remains employed (with probability $1-\alpha^{i}$ ). As the agent is employed, his marginal utility is 1 . The second term at the right hand side is the valuation of the same return when the agents is unemployed. In this case, his marginal utility is $u^{\prime}\left(\delta+\left(P_{t+1}+y_{t+1}\right) x_{t}^{i}\right)$, where $x_{t}^{i}$ is the quantity of assets bought in period $t$, because the agent liquidates all financial holdings to consume.

From the previous equation, we deduce that the asset price and the asset quantity chosen by employed agents only depends on their type and on aggregate parameters, and not on their previous wealth. With a slight abuse of notation, we note them respectively $P_{t}$ and $x_{t}^{i}$. Moreover, this price $P_{t}$ and the asset quantities $x_{t}^{i}$ depend only on the current aggregate state $y_{t}$, and not on the whole history of shocks. We simply note them respectively $P_{k}$ and $x_{k}^{i}$ for the type $i$ agent in the state of the world $k=G, B$.

For each type of agents $i=1,2$ there are four different classes of households in each period $t$ : (i) Unemployed agents at date $t$, who were employed before. These agents denoted eu consume the amount $\delta+\left(P_{t}+y_{t}\right) x_{t-1}^{i}$; (ii) Unemployed agents, who were unemployed in the previous period. These agents denoted $u u$ consume the amount $\delta$; (iii) Employed agents ee, who were employed before and (iv) Employed agents ue, who were unemployed before. Agents belonging to these last two classes consume the same amount $u^{\prime-1}(1)$ and buy the same quantity of asset $x_{t}^{i}$. However, they supply a different quantity of labor, because their beginning-of-period wealth is different.

\subsubsection{Proof of the existence of the equilibrium}

The previous equilibrium exists if: (i) Unemployed agents are credit constrained, and if (ii) Both agents' types trade in asset markets. First sufficient conditions are therefore that Euler equations of $e u$ and $u u$ agents for both types hold with strict inequality, reflecting that the asset is too

expensive for them. Since the quantity of assets $x_{h}^{i}$ held in state $h=B, G$ in the beginning of the period by the type $i$ agent $e u$, is positive, it is sufficient to prove that eu agents are credit 
constrained, which implies that $u u$ also are. This simply expresses in the state $k=B, G$ as:

$$
P_{k} u^{\prime}\left(\delta+\left(P_{k}+y_{k}\right) x_{h}^{i}\right)>\beta \sum_{j=G, B} \pi_{k, j} u^{\prime}(\delta)\left(P_{j}+y_{j}\right)+\pi_{k, j} \rho^{i}\left(1-u^{\prime}(\delta)\right)\left(P_{j}+y_{j}\right)
$$

The second condition for the equilibrium to exist is that the valuation of the asset by employed agents of both types is similar. Using equation 12 , we deduce that in state $k=G, B$ :

$\alpha^{1} \sum_{j=G, B} \pi_{k, j}\left(u^{\prime}\left(\delta+\left(P_{j}+y_{j}\right) x_{k}^{1}\right)-1\right)\left(P_{j}+y_{j}\right)=\alpha^{2} \sum_{j=G, B} \pi_{k, j}\left(u^{\prime}\left(\delta+\left(P_{j}+y_{j}\right) x_{k}^{2}\right)-1\right)\left(P_{j}+y_{j}\right)$

This equation states that both types of agents value similarly the asset when falling into unemployment.

The proof of the existence of the equilibrium is done is appendix. The following proposition summarizes the result.

Proposition 1 (Equilibrium existence) A unique equilibrium exists in an homogeneous economy without aggregate shocks $\left(\alpha^{1}=\alpha^{2}=\alpha\right.$ and $\left.y_{G}=y_{B}\right)$ as soon as the following condition holds:

$$
1<u^{\prime}(\delta)<1+\frac{1-\beta}{\beta \alpha}
$$

Moreover, the equilibrium exists is still unique when 1) the tree size $V$ is not too large, 2) heterogeneity in $\alpha$ is small and 3) the aggregate uncertainty remains limited.

The intuition of the proposition is the following. The condition (11) is a necessary condition for the equilibrium to exist when there is no aggregate risk and when agents have the same idiosyncratic risk. In this case, one can simply derive the price of the asset $P$ and check unemployed agents are credit constraints. Both inequalities of the condition are compatible since $\beta<1$. We will assume that the condition hodls in the remaining of the paper. The condition is all the less severe since the probability $\alpha$ of falling into unemployment is small. This condition also allows us to rule out sunspot equilibria and to be sure that the equilibrium we consider is unique. Contrary to the paper of Bowman and Faust (1997), options cannot play any role in our economy when the larket is complete for the aggregate risk before the option introduction.

In the case with risk, the intuition is simply that even an agent endowed with all available assets is unable to unwind his credit constraint when falling into unemployment in a good state of the world. The restriction on the aggregate uncertainty insures that the portfolio liquidation value is not too high in any state of the world. Finally, the agents' probabilities $\alpha^{i}(i=1,2)$ of falling into unemployment must be not too different from each other, for each of them to desire to hold the asset in equilibrium. 


\subsection{Interaction between aggregate uncertainty and heterogeneity}

As prices and quantities depend only on the aggregate state, the Euler equation (8) defining the asset price in state $k=B, G$ can be expressed as:

$$
P_{k}=\beta \sum_{j=G, B} \pi_{k, j}\left(1+\alpha^{i}\left(u^{\prime}\left(\delta+\left(P_{j}+y_{j}\right) x_{k}^{i}\right)-1\right)\right)\left(P_{j}+y_{j}\right), i=1,2, k=G, B
$$

Eq. (12) summarizes four different equations, stating that the asset valuation is the same for both agents' types $i=1,2$ in both states of the world $k=G, B$.

The market clearing condition in each state of the world expresses simply as follows:

$$
V=\eta^{1} x_{k}^{1}+\eta^{2} x_{k}^{2}, k=G, B
$$

The equilibrium consists now in the set of six endogenous variables $\left\{P_{G}, P_{B}, x_{G}^{1}, x_{B}^{1}, x_{G}^{2}, x_{B}^{2}\right\}$ defined by the six equations $(12)$ and $(13)$. It is now easy to study the interaction between aggregate uncertainty and heterogeneity. Before presenting the main result of this section, we have to make an additional assumption about the shape of the utility function.

Assumption D (Utility function) We assume that the following inequality holds in the equilibrium without aggregate risks $\left(y_{B}=y_{G}=y\right)$ :

$$
-x^{1} \frac{u^{\prime \prime}\left(\delta+x^{1}(P+y)\right)}{u^{\prime}\left(\delta+x^{1}(P+y)\right)-1}>-x^{2} \frac{u^{\prime \prime}\left(\delta+x^{2}(P+y)\right)}{u^{\prime}\left(\delta+x^{2}(P+y)\right)-1}
$$

where $P$ is the price and $x^{1}$ and $x^{2}$ the asset quantities chosen by agents 1 and 2 in the economy without aggregate risk.

Solving for the price $P$ and quantities $x^{1}$ and $x^{2}$, the previous inequality becomes a condition on the shape of the utility function. This condition always holds for standard utility classes, as CRRA, CARA or quadratic utilities, and is thus quite general. This condition states that the agents 1 who has the biggest probability to fall into unemployement $\left(\alpha^{1}>\alpha^{2}\right)$ benefit relatively more from an increase in the return on the financial asset. To see this, note that the function of $P+y$ defined by $u^{\prime}\left(\delta+x^{i}(P+y)\right)-1$ is the difference in marginal utilities between employed and unemployed workers. The condition states that the elasticity of this function with respect to $P+y$ is higher for agents 1 than for agents 2, which means that the difference in marginal utilities between employed and unemployed is proportionally higher for agents 1 than for agents 2 . The following proposition summarizes the results about the effect of aggregate shocks.

Proposition 2 (Aggregate shock effects) In the vicinity of the equilibrium without uncertainty $\left(y_{B}=y_{G}=y\right)$, 
(i) The agents, who are more likely to fall into unemployment $\left(\alpha^{1}>\alpha^{2}\right)$ purchase in both states of the world a larger asset quantity than the other ones: $x_{k}^{1}>x_{k}^{2}>0$ for $k=B, G$.

(ii) Type 1 agents hold less assets in the good state of the world than in the bad one: $x_{G}^{1}<x_{B}^{1}$, while the reverse holds for type $2: x_{G}^{2}>x_{B}^{2}$.

First, as the demand for insurance against the risk of falling into unemployment by agents 1 is higher than the one of agents 2, they hold more asset in all states of the world. Second, as the aggregate state is persistent, the average return on the asset is higher in the good state of the world than in the bad state of the world. As type 1 agents demand the asset to self-insure themselves, they need to demand less asset in good state to obtain the same expected self-insurance. The same is true for agents 2, but one can show that Assumption $\mathrm{D}$ implies that the equilibrium quantities are driven by the behavior of type 1 agents, who are more sensitive to the return on the risky asset. In consequence, agents 2 hold less assets in state $B$ than in state $G$.

The next proposition summarizes the main results according to a change in the idiosyncratic risk.

Proposition 3 (Heterogeneity effects) In the vicinity of the symmetric equilibrium $\left(\alpha^{1}=\alpha^{2}\right)$, a larger unemployment risk for the type 1 agents (a larger $\alpha^{1}$ ) yields the following consequences:

(i) The asset price increases in both states of the world, but more in the good one than in the bad one.

(ii) The asset holdings of the type 1 agents also increase in both states of the world, but more in the bad state than in the good one.

The previous proposition considers the case where type 1 agents experience a larger $\alpha^{1}$ and thus a more severe risk than agents of type 2. Facing a larger probability of unemployment, these agents need to better hedge against that risk, which raises their asset demand. This larger demand increases asset prices in both states of the world, but not homogeneously. Since aggregate states are persistent, purchasing the asset in the bad state of the world is mainly purchasing a hedge against unemployment in the bad state, when the asset pays off badly and is less effective in helping to overcome credit constraints. In consequence, the asset price increases less in the bad state than in the good one. A larger probability of unemployment therefore raises both prices, but also their variance.

Type 1 agents, who experience a larger individual risk, need to better self-insure themselves, and therefore demand a larger asset quantity in both states of the world. However, due to Assumption D with the same quantity of assets, they will be much better insured in the good state of the 
world. In consequence, they will purchase more assets in both states of the world, but more in the bad state than in the good one. The variance of their holdings will also therefore increase.

We have presented in this section the results in the case of market incompleteness for the aggregate shock. In the next section, we introduce options and study the exchange of insurance in the business cycle.

\section{The economy with a risky asset and a derivative call option}

In this section, we complete the credit market of the preceding economy by introducing a derivative asset, whose underlying is the risky tree. More precisely, agents are allowed to trade a supplementary security, which is a call option with a maturity of one period and a strike contained between the asset price in the bad state of the world and the one in the good state. The call is therefore designed in order to only payoff in the good state of the world. The market is complete for the aggregate risk, while it is still incomplete regarding the idiosyncratic risk and while credit constraints still limit agents' investment sets.

In addition to tree shares, which are in positive supply, agents trade options, which are in zero net supply. Since agents of different types have different risk appetites, they are willing to exchange insurance between themselves through the trade of positive quantities of options, in order to smooth out their portfolio payoffs across states of the world.

Let us now turn to the formal description of the model.

\subsection{Description of the economy}

Except the option introduction, the economy remains unchanged compared to the previous one. Since prices are endogenous, it is not necessarily obvious to find a strike $K$, contained between the asset prices in both states of the world: $P_{B}<K<P_{G}$. The simplest solution consists in first introducing options in a homogeneous economy, where both agents face a similar unemployment risk. Because they are perfectly symmetric, agents hold similar portfolios. In consequence, introducing the option in zero net supply implies that the call is priced but not traded. Asset prices in both states of the world are different (see Proposition 2) and we can pick up a strike between both assets prices. In a second step, increasing the heterogeneity between agents continuously move asset prices and we deduce that it remains possible to find such a strike.

We note $s_{t}^{i}$ the quantity of calls that an agent of type $i$ purchases at the price $Q_{t}$ in period $t$. The type $i$ agent's program consists in choosing his consumption level, his labor supply, and his asset and option demands in order to maximize his intertemporal utility subject to a budget constraint (including option purchases and payoffs) and a credit constraint stating that the agents' 
financial wealth must remain positive. Noting $E_{0}$ the unconditional expectation over aggregate and idiosyncratic uncertainty, the program of the agent of type $i$ expresses as follows:

$$
\begin{array}{ll} 
& \max _{\left\{c_{t}, l_{t}, x_{t}, s_{t}\right\}_{t=0,1, \ldots}} E_{0} \sum_{t=0}^{\infty} \beta^{t}\left(u\left(c_{t}^{i}\right)-l_{t}^{i}\right) \\
\text { s.t. } \quad & c_{t}^{i}+P_{t} x_{t}^{i}+Q_{t} s_{t}^{i}=e_{t}^{i} l_{t}^{i}+\left(1-e_{t}^{i}\right) \delta+\left(P_{t}+y_{t}\right) x_{t-1}^{i}+\left(P_{t}-K\right)^{+} s_{t-1}^{i} \\
& P_{t} x_{t}^{i}+Q_{t} s_{t}^{i} \geq 0 \\
& c_{t}^{i}, l_{t}^{i} \geq 0 \\
& \lim _{t \rightarrow \infty} \beta^{t} u^{\prime}\left(c_{t}^{i}\right) x_{t}^{i}=\lim _{t \rightarrow \infty} \beta^{t} u^{\prime}\left(c_{t}^{i}\right) s_{t}^{i}=0
\end{array}
$$

The agent objective is the maximization of his intertemporal utility function (14) under the set of constraints $15-18$. The first constraint 15$]$ is the budget constraint at date $t$ and equalizes agent's resources (labor income, or domestic production, and revenue from the sale of both financial securities) to his expenditure (consumption and security purchases). The inequality 16 is the borrowing constraint of the agent imposing that his financial wealth must remain positive. The two other constraints, positivity of consumption and labor in 17 ) and transversality conditions in (18) are technical and will always be satisfied in the equilibrium we will consider.

It is noteworthy that the condition (16) allows agent to "issue" options, at least up to certain extent. Due to the heterogeneity and the zero net volume, there will be a positive exchange of options at the equilibrium and the option purchased by an agent needs to be issued and sold by another agent. The quantity of traded options is also called the open interest and measures the activity in the option market:2

\subsection{The equilibrium}

\subsubsection{Euler equations}

As in the previous economy, we build an equilibrium with four agents' classes for each type, where classes depend on the present and past employment status of agents. Unemployed agents $e u$ do not participate to financial markets and once they fall into unemployment and liquidate their portfolio, they remain credit constrained. Their financial wealth is supposed to be never sufficient to overcome borrowing constraints. Liquidating assets and options, they consume in state $j=B, G$ (while the previous state is denoted $k=B, G) \delta+\left(P_{j}+y_{j}\right) x_{k}^{i}+\left(P_{j}-K\right)^{+} s_{k}^{i}$. Long lasting unemployed agents $u u$ only consume their domestic production $\delta$. Employed agents ue and $e e$ of both types $i=1,2$ participate to both the asset and the option financial markets. Since they are infinitely

\footnotetext{
${ }^{2}$ In our economy, traded volumes and open interest are exactly similar, since every option contract is exactly traded once during the period.
} 
elastic in labor, all employed agents of the same type consume the same amount $u^{\prime-1}(1)$ and hold the same financial portfolio. These agents only differ along their labor effort. As in the no option equilibrium, prices and quantities only depend on the current aggregate state and not on the whole history. We deduce the following Euler equations for the asset and the option, as well as the corresponding market clearing condition $(k=B, G$ and $i=1,2)$ :

$$
\begin{aligned}
P_{k} & =\beta \sum_{j=G, B} \pi_{k, j}\left(1+\alpha^{i}\left(u^{\prime}\left(\delta+\left(P_{j}+y_{j}\right) x_{k}^{i}+\left(P_{j}-K\right)^{+} s_{k}^{i}\right)-1\right)\right)\left(P_{j}+y_{j}\right) \\
Q_{k} & =\beta \pi_{k, G}\left(1+\alpha^{i}\left(u^{\prime}\left(\delta+\left(P_{G}+y_{G}\right) x_{k}^{i}+\left(P_{G}-K\right)^{+} s_{k}^{i}\right)-1\right)\right)\left(P_{G}-K\right) \\
V & =\eta^{1} x_{k}^{1}+\eta^{2} x_{k}^{2} \\
0 & =\eta^{1} s_{k}^{1}+\eta^{2} s_{k}^{2}
\end{aligned}
$$

The pricing kernel has a similar interpretation as in the economy without options. A first component refers to the consumption smoothing between two employment periods. The second term reflects the hedging against the unemployment risk. The security is all the more valued since it is helpful in insuring against this risk and since the expected cost of unemployment is high for the agent. The cost of unemployment is measured as the (inframarginal) variation of marginal utility experienced by the agent when falling into unemployment, which is $u^{\prime}\left(\delta+\left(P_{j}+y_{j}\right) x_{k}^{i}+\left(P_{j}-K\right)^{+} s_{k}^{i}\right)-1$ if $k$ and $j$ denotes respectively the previous and the current states. This expected cost is large when (i) the probability of losing your job is high and (ii) the portfolio liquidation value that the agent consumes when unemployed is small.

There are however two main differences with the previous economy. First, obviously, there is an additional security and therefore a supplementary Euler equation 20 . Second, the option only payoffs in the good state of the world. The Euler equation only mentions a term for the good state of the world. Moreover, options only matter in the liquidation component of the Euler equation in the good state of the world.

As a conclusion, an equilibrium in this economy consists in the set of four prices $\left\{P_{k}, Q_{k}\right\}_{k=B, G}$ and eight security quantities $\left\{x_{k}^{i}, s_{k}^{i}\right\}_{k=B, G}^{i=1,2}$, such that the twelve equations $19-22$ ( $k=B, G$ and $i=1,2)$ hold.

\subsubsection{Equilibrium existence conditions}

The previous equilibrium with four agents' categories for each type $i=1,2$ exists as long as (i) employed agents of both types participate to financial markets and (ii) unemployed agents are credit-constrained when falling into unemployment.

The first condition implies that the agents' valuation for both securities is the same. The equality of expected unemployment costs for both agents therefore expresses in each state of the 
world $B$ and $G$, where $h=B, G$ denotes the previous state:

$$
\begin{gathered}
\alpha^{1}\left(u^{\prime}\left(\delta+\left(P_{G}+y_{G}\right) x_{h}^{1}+\left(P_{G}-K\right) s_{h}^{1}\right)-1\right)=\alpha^{2}\left(u^{\prime}\left(\delta+\left(P_{G}+y_{G}\right) x_{h}^{2}+\left(P_{G}-K\right) s_{h}^{2}\right)-1\right), h=G, B \\
\alpha^{1}\left(u^{\prime}\left(\delta+\left(P_{B}+y_{B}\right) x_{h}^{1}\right)-1\right)=\alpha^{2}\left(u^{\prime}\left(\delta+\left(P_{B}+y_{B}\right) x_{h}^{2}\right)-1\right), h=G, B
\end{gathered}
$$

We will use later on these equations to investigate the portfolio holdings of both agents.

The second condition for the equilibrium to exist implies that both asset and option prices are too high for unemployed agents to purchase them. Agents $e u$, who have just lost their jobs, hold at the beginning of the period a financial portfolio and are wealthier than $u u$. If the former do not participate to financial markets because of too high prices, the latter will also be excluded. The two following inequalities, one for each security market, insure that none of unemployed agents trade any security:

$$
\begin{aligned}
& P_{k} u^{\prime}\left(\delta+\left(P_{k}+y_{k}\right) x^{i}+\left(P_{k}-K\right)^{+} s^{i}\right)>\beta \sum_{j=G, B} \pi_{k, j}\left(u^{\prime}(\delta)+\rho^{i}\left(1-u^{\prime}(\delta)\right)\right)\left(P_{j}+y_{j}\right) \\
& Q_{k} u^{\prime}\left(\delta+\left(P_{k}+y_{k}\right) x^{i}+\left(P_{k}-K\right)^{+} s^{i}\right)>\beta \pi_{k, G}\left(u^{\prime}(\delta)+\rho^{i}\left(1-u^{\prime}(\delta)\right)\right)\left(P_{G}-K\right)
\end{aligned}
$$

Using expressions of prices $P$ and $Q$, we deduce from (25) and (26) two conditions, which are very similar to 9 for the equilibrium without options. In consequence, unemployed agents will not trade neither options, nor assets, as long as we remain in the vicinity of the equilibrium with small asset volumes and without heterogeneity nor aggregate uncertainty.

We can therefore state a similar proposition to Proposition 1 of the previous section.

Proposition 4 (Equilibrium existence) We suppose that the condition 111) holds. A unique equilibrium with limited heterogeneity and option trading exists as soon as the tree size $V$ is not too large and the heterogeneity and the aggregate uncertainty remain limited.

The proof strategy is exactly the same as in the no option economy and the formal proof is left in appendix.

\subsection{Interaction between aggregate uncertainty and heterogeneity}

We analyze now the interaction between uncertainty and heterogeneity along two dimensions. We first discuss the portfolio compositions, and then how these holdings, as well as security prices are affected by the aggregate uncertainty. In the next section, we focus on welfare consequences of the option introduction.

We suppose that Assumption $\mathrm{D}$ still holds. The larger the asset quantity an agent holds, the more he will benefit from a rise in asset prices when falling into unemployment. Key equations determining agents' holdings are both the market clearing conditions 21) and 22, as well as 
equations (23) and (24), which insure that both agents' types trade positive quantities of both securities. More precisely, the equation (24) shows how agents chose their asset quantity in order to hedge themselves against unemployment in the bad state of the world. The equation 23 describes how agents chose their option quantity, so as to adjust their insurance against unemployment in the good state of the world. In a nutshell, the asset matters for hedging in the bad state, while option matters in the good one.

The following proposition sums up the results about the portfolio composition.

Proposition 5 (Agents' portfolios) In the economy with a call option, agents' portfolios exhibit the following features:

(i) Asset and option quantities do not depend on the state of the world and are noted respectively $x^{i}$ and $s^{i}$ for type $i=1,2$ agents.

(ii) Agents of type 1, who face a larger risk of unemployment choose to hold a larger quantity of assets than type 2 agents: $x^{1}>x^{2}>0$, and the former agents sell options to the latter: $s^{1}<0<s^{2}$.

The intuition for the first result is the following. Since the option only payoffs in the good state of the world, both agents need to value analogously the option when falling into unemployment in the good state of the world. In consequence, the expected cost of unemployment for both agents' types has to be the same in the good state of the world. The asset payoffs in both states of the world but due to the preceding remark, agents need to value the asset similarly in the bad state of the world: The expected cost of unemployment in the bad state has also to be identical for both agents' types. In addition to the market clearing conditions, one can show that these equalities between expected unemployment costs in each state of the world imply that portfolio liquidation values do not depend on the state of the world, neither do the security quantities. This is the consequence of the complete market assumption.

The intuition for the second result of Proposition 5 is the following. Type 1 agents, who are the more likely to fall into unemployment purchase a larger quantity of assets to self-insure against the risk of falling into unemployment in the bad state of the world. Without options, agents of type 1 have insured against unemployment in the bad state of the world through the purchase of assets. In the good state of the world, the asset becomes a better insurance device (because of a higher sale price and a higher dividend), which benefits more to type 1 agents who hold a larger asset quantity (due to Assumption D). In consequence, type 1 agents hold a portfolio that payoffs too much in the good state of the world: They therefore sell options, in order to decrease their portfolio liquidation value in the good state of the world. On the other side, type 2 agents have 
purchased a smaller quantity of assets and are not sufficiently insured in the good state of the world against unemployment (Assumption $\mathrm{D}$ again). The type 2 agent needs additionally to purchase options to improve his hedging in the good state of the world, and to decrease the expected cost of unemployment in that state of the world. It is noteworthy that type 2 agents purchase a positive quantity of assets, at least when the heterogeneity is not too strong $3^{3}$ Both agents' types therefore optimally choose not to "issue" tree shares, which are the unique insurance against unemployment in the bad state.

From a financial point of view, type 1 agents choose a sort of delta hedging strategy at the equilibrium, in the sense that they optimally chose a portfolio composition, which is less affected by the variation of the underlying than their portfolio without options was.

This result is consistent with the result of Franke, Stapleton, and Subrahmanyam (1998), who prove that agents with low or no uninsurable risk have a concave sharing rule, which means that they sell insurance to high risk agents in the good state of the world. Indeed, the return on the portfolio holdinds is proportionnaly lower in the good state of the world. This return is obtained in our setup by low risk agents buying call options.

We now explain how the aggregate uncertainty may affect security holdings and prices. This is the main theoretical result concerning the volume of options in the business cycle.

Proposition 6 (Aggregate uncertainty effects) In the vicinity of the uncertain equilibrium $\left(y_{G}=y_{B}\right)$, an increase in aggregate uncertainty has the following consequences:

(i) The asset price increases in the good state and decreases in the bad one, while the average price remains unchanged.

(ii) The option price increases in both states, but more in the good one that in bad one.

(iii) The more volatile the dividends, the larger the option traded volume.

The intuition for these results is the following. We consider a mean preserving spread of dividends. In that case, the aggregate uncertainty increases: the bad state become relatively worst, while the good state becomes better. In consequence, the quality of securities as an insurance device evolves. While the quality of assets in the bad state of the world deteriorates (because of the aggregate state persistence, it will pay off badly with a large probability), the quality of assets in the good state of the world and the quality of options in both states appreciate. The spread between prices in both states increases with the uncertainty, even if the average price of the asset is not modified (result (i)). Regarding the option, its price increases in both states, but more in

\footnotetext{
${ }^{3}$ More precisely, we must have at the uncertain equilibrium: $\alpha^{1} u^{\prime}(\delta+(P+y) V)<\alpha^{2} u^{\prime}(\delta)$, which holds as soon as the heterogeneity remains limited.
} 
the good one than in the bad one. As the price of the underlying asset increases in the good state of the world, the return on the option, which is $\left(P_{G}-K\right)^{+}$increases in the good state (recall that the retun on the option is 0 in the bad state of the world). As a consequence, the price of the option increases. Moreover, as the aggregate states are persistent, this increase is higher in the good state of the world (result (ii)).

This mean spread of dividends also impacts portfolio compositions. Due to a smaller dividend in the bad state, type 1 agents, who are the more likely to fall into unemployment, would like to purchase a larger quantity of assets to obtain a sufficient hedging. Oppositely, a rise in the dividend in the good state of the world gives them an incentive to purchase less assets. On the whole, the overall effect remains positive since their first concern lies in the insurance in the bad state of the world: More volatile dividends yield a larger asset purchase by type 1 agents. These type 1 agents consequently sell a larger quantity of options in order to decrease their insurance level in the good state of the world, while type 2 agents purchase these options in order to improve their hedging in the good state of the world.

In consequence, more volatile dividends yield more volatile asset prices, more volatile (and on average higher) option prices, and a larger quantity of traded options (result (iii)). Our model is therefore able to reproduce the stylized fact stating that the open interest of options, i.e. the number of open contracts, which is similar to $\left|s^{1}\right|$ in our economy, increases when asset prices become more volatile. This fact is notably reported in Buraschi and Jiltsov (2006).

The degree of market incompleteness has also consequences on the portfolio compositions, as it was the case in the no-option economy. The results are given in the next proposition.

Proposition 7 (Heterogeneity effects) In the vicinity of the symmetric equilibrium $\left(\alpha^{1}=\alpha^{2}\right)$, a larger unemployment risk for the type 1 agents (i.e. a larger $\alpha^{1}$ ) has the following consequences:

(i) The price of both securities increase in both states of the world, but more in the good one than in the bad one.

(ii) The type 1 agents purchase more assets and sell more options.

A larger heterogeneity raises therefore the volume of traded options.

We now consider an increase in the unemployment risk for type 1 agents (i.e. a larger $\alpha^{1}$ ) in the vicinity of the symmetric equilibrium $\left(\alpha^{1}=\alpha^{2}\right)$. Type 1 agents experience a higher probability of falling into unemployment, which raises their expected cost of unemployment. Agents express therefore a larger demand for self-insurance, which yields larger prices for both securities. The price increase is more important in the good state of the world, when both securities are a better hedge against unemployment, due to the aggregate state persistence. To sum up, a larger risk of 
unemployment increases the average security price, but also the price dispersion (result (i)). The increase in the demand for insurance by agents 1 translates into a higher demand for the asset to insure in the bad state of the world and a higher quantity of options sold to insure in the good state of the world, as the option provides only insurance in the good state of the world.

\subsection{Effects of the option introduction}

We analyze the effects of an option introduction, starting from an economy without aggregate risk, and where we increase progressively the risk through a mean preserving spread of dividends. Introducing the option modifies portfolios' payoffs ex post in each state of the world. In the economy without option, agents only had the opportunity to purchase a single asset to hedge them against the risk of unemployment in an "average" state of the world. With the option completing the market, type 1 agents facing a high idiosyncratic risk insure themselves better in the bad state of the world and less in the good state of the world, where their unemployment cost decreases more than the one of the other type because they hold a larger asset quantity. In consequence, in the economy with option, their portfolio will pay off more in the bad state of the world, while less in the good state. They better smooth their portfolio payoffs across states of the world. It is moreover noteworthy that expected portfolio payoffs (tomorrow, contingent in being in a given state today) are similar in both economies. In fact, asset quantities in the economy without options are chosen, such as to replicate these expected payoffs.

We now turn to the impact of the option introduction on the ex ante welfare. We analyze the impact again in the vicinity of the equilibrium without aggregate shocks.

Proposition 8 (Impact of options on the welfare) The option introduction redistributes welfare from agents facing a small risk of unemployment to agents facing a larger risk.

At the first order, the welfare is impacted through (i) the modification of portfolio composition, while keeping prices equal to prices without aggregate shock and through (ii) the modification of prices, while keeping quantities equal to the ones without aggregate shocks.

The intuition can be obtained through variations of portfolio compositions, because the price of the asset is not impacted at the first order by the introduction of options. Since both assets are in fixed supply, the portfolio compositions of both agents' types evolve in opposite directions. This has two important consequences. First, the aggregate ex ante welfare is not impacted at the first order when the option is introduced. Second, one of both agents' types will benefit from the option introduction, while the other will suffer from it. Agents, who are the more likely to fall into unemployment have the opportunity to better self-insure themselves with options: their portfolio pays off more in the bad state, while less in the good one. They are able to better smooth out 
their portfolio liquidation values. In consequence, they benefit from the option introduction, which allows them for a better self-insurance. On the opposite, the other agents' type suffers from the option introduction, which deters their insurance ex post. The option introduction allows a welfare redistribution from agents facing a small risk of unemployment to agents facing a larger risk.

The option introduction is therefore not Pareto improving, which illustrates Elul (1995) and Cass and Citanna (1998) papers about the impact of financial innovation on welfare. These authors find the introduction of a new asset is not always Pareto improving. In our case, the result stems from change in the volatility of agents' portfolios.

We have described in details the economy with two agents' types and two aggregate states. We now generalize our equilibrium to an economy with arbitrary large numbers of agents' types, of aggregates states, and of call options.

\section{Generalization}

We have restricted our attention sofar to a simple economy where options are traded. In fact, it was the simplest economy, in which options could be traded: the aggregate shock takes only two values and the heterogeneity is limited to two agents' types. The introduction of a single option completes the market for the aggregate risk. The simplicity and the tractability of pricing equations in this framework has allowed us to derive analytical results regarding the interaction of aggregate uncertainty and heterogeneity and its impact on security prices and agents' portfolios.

However, we can prove in a more general setup that an equilibrium exists, where options are traded. We extend the previous economy along three dimensions. First, we suppose that the dividend, i.e. the aggregate risk may take $n \geq 1$ values denoted $\left\{y_{1}, \ldots, y_{n}\right\}$. The dividend evolves again like a first order Markov chain and the probability of transition from state $k$ to state $l$ $(1 \leq k, l \leq n)$ is noted $\pi_{k, l}$, with obviously $\sum_{l=1}^{n} \pi_{k, l}=1$.

Second, we suppose that there are $I \geq 1$ types of agents, facing different probabilities to fall into and to leave unemployment that we note respectively $\alpha^{i}$ and $\rho^{i}$, with $i=1, \ldots, I$. The population of each type is normalized to 1 and the proportion of employed agents of type $i$ is $\eta^{i}=\frac{1-\rho^{i}}{2-\alpha^{i}-\rho^{i}}$.

Finally, we suppose that the productivity of agents depends both on the agent's type and on the aggregate state. We note $w_{k}^{i}$ the productivity of agent with type $i=1, \ldots, I$ and in the state $k=1, \ldots, n$.

After studying the existence of a limited heterogeneity equilibrium without options, we introduce $L \geq 1$ call options with a one period maturity and a strike $K_{l}(l=1, \ldots, L)$, such that the market is not necessary complete for the aggregate risk after the option introduction. We then prove the existence for the economy with options and necessary conditions for the trade of options. 


\subsection{The economy without options}

As in the simple two state economy, we conjecture the existence of a limited heterogeneity equilibrium, where employed agents of all types trade the asset, while unemployed agents are kept out of financial markets. For each type, we suppose again that there are four classes of agents, which depend on the past and current employment status.

The conjectured equilibrium is characterized by the $n I$ Euler equations of the employed agents (one per agent and in each aggregate state), the $n$ market clearing conditions (one in each state) and the $n I$ Euler inequalities keeping $e u$ agents (and a fortiori the poorest $u u$ ) out of the financial market. In state $k=1, \ldots, n$, all employed agents of the same type $i=1, \ldots, I$ consume the same amount $u^{\prime}\left(1 / w_{k}^{i}\right)$ and purchase the same quantity of assets $x_{k}^{i}$. We note $P_{k}$ the price of one unit of tree in that state $k$. Unemployed agents consume their domestic production $\delta$ and possibly the sale value of their financial portfolio.

Equations and inequalities characterizing the equilibrium are $(k, h=1, \ldots, n$ and $i=1, \ldots, I)$ :

$$
\begin{aligned}
P_{k} & =\beta \sum_{j=1}^{n} \pi_{k, j} \frac{w_{k}^{i}}{w_{j}^{i}}\left(1+\alpha^{i}\left(w_{j}^{i} u^{\prime}\left(\delta+\left(P_{j}+y_{j}\right) x_{k}^{i}\right)-1\right)\right)\left(P_{j}+y_{j}\right) \\
V & =\sum_{i=1}^{I} \eta^{i} x_{k}^{i} \\
P_{k} u^{\prime}\left(\delta+\left(P_{k}+y_{k}\right) x_{h}^{i}\right) & >\beta \sum_{j=1}^{n} \pi_{k, j}\left(\rho^{i} \frac{1}{w_{j}^{i}}+\left(1-\rho^{i}\right) u^{\prime}(\delta)\right)
\end{aligned}
$$

Using the implicit function theorem, we extend the equilibrium existence of the previous section to this much more general framework.

Proposition 9 (Equilibrium existence) We suppose that the condition 11) still holds. In the general case without options, a unique equilibrium where unemployed agents of all types are excluded from financial markets while employed agents of all types trade strictly positive quantities of assets, exists as soon as the tree size $V$ is not too large and the heterogeneity in $\alpha$ and the aggregate uncertainty remain limited.

\subsection{Economy with options}

We introduce in the previous economy, $L$ non redundant call options maturing in one period. We proceed in three steps.

First, for introducing non-redundant options, we need to be sure that asset prices are different in some states of the world. To make it clear, we consider the economy without option, with no idiosyncratic shocks and with the dividend as the single source of aggregate risk. The vector of price $P=\left(P_{1}, \ldots, P_{n}\right)$ expresses in that case as a linear function of the dividend vector $y=\left(y_{1}, \ldots, y_{n}\right)$, 
such that $P=\left(I_{n}-\beta \pi\right)^{-1} y$, where $\pi=\left(\pi_{k l}\right)_{1 \leq k, l \leq n}$ is the matrix of transition probabilities. In consequence, as soon as we depart from the no aggregate shock equilibrium (while in its vicinity), asset prices are different in at least two states of the world and different from each other, except on a set of measure null 4

Second, the continuity result in the no-option economy implies that prices remain different, even if we allow for idiosyncratic shocks and for another source of aggregate uncertainty, but where agents are homogeneous.

Finally, we are now able to introduce options, such that at most one strikes lies between two different asset prices. So, if there are $h$ different prices supposedly ranked, $P_{1}<\ldots<P_{h}$, it is possible to introduce $h-1$ different calls, whose strikes are as follows $P_{1}<K_{1}<P_{2}<\ldots<$ $P_{h-1}<K_{h-1}<P_{h}$. In this economy, options are priced, but not traded.

We now need to prove that introducing heterogeneity does not modify the equilibrium existence. As in the no-option economy, the conjecture equilibrium is such that: (i) employed agents of all types participate to the $L+1$ financial markets, and (ii) unemployed agents do not trade any security. In state $k=1, \ldots, n$, an employed agent of type $i=1, \ldots I$ purchases the quantity $s_{k, l}^{i}$ of options with a strike $K_{l}(l=1, \ldots, L)$ at a price $Q_{k, l}$.

With options, the equilibrium is characterized by $n I(L+1)$ Euler equations $(L+1$ financial securities priced in $n$ states of the world where $I$ agents' types participate), $n(L+1)$ market clearing conditions $(L+1$ markets) and $n I(L+1)$ inequalities characterizing the equilibrium are $(k, h=1, \ldots, n ; m=1, \ldots, L$ and $i=1, \ldots, I)$ :

$$
\begin{aligned}
P_{k} & =\beta \sum_{j=1}^{n} \pi_{k, j} \frac{w_{k}^{i}}{w_{j}^{i}}\left(1+\alpha^{i}\left(w_{j}^{i} u^{\prime}\left(\delta+\left(P_{j}+y_{j}\right) x_{k}^{i}+\sum_{l=1}^{L}\left(P_{j}-K_{l}\right)^{+} s_{k}^{i}\right)-1\right)\right)\left(P_{j}+y_{j}\right) \\
Q_{k, m} & =\beta \sum_{j=1}^{n} \pi_{k, j} \frac{w_{k}^{i}}{w_{j}^{i}}\left(1+\alpha^{i}\left(w_{j}^{i} u^{\prime}\left(\delta+\left(P_{j}+y_{j}\right) x_{k}^{i}+\sum_{l=1}^{L}\left(P_{j}-K_{l}\right)^{+} s_{k}^{i}\right)-1\right)\right)\left(P_{j}-K_{m}\right)^{+} \\
V & =\sum_{i=1}^{I} \eta^{i} x_{k}^{i} \\
0 & =\sum_{i=1}^{I} \eta^{i} s_{k, m}^{i} \\
P_{k} & >\beta \frac{1}{u^{\prime}\left(\delta+\left(P_{k}+y_{k}\right) x_{h}^{i}+\sum_{l=1}^{L}\left(P_{k}-K_{l}\right)^{+} s_{h}^{i}\right)} \sum_{j=1}^{n} \pi_{k, j}\left(\rho^{i} \frac{1}{w_{j}^{i}}+\left(1-\rho^{i}\right) u^{\prime}(\delta)\right) \\
Q_{k, m} & >\beta \frac{1}{u^{\prime}\left(\delta+\left(P_{k}+y_{k}\right) x_{h}^{i}+\sum_{l=1}^{L}\left(P_{k}-K_{l}\right)^{+} s_{h}^{i}\right)} \sum_{j=1}^{n} \pi_{k, j}\left(\rho^{i} \frac{1}{w_{j}^{i}}+\left(1-\rho^{i}\right) u^{\prime}(\delta)\right)
\end{aligned}
$$

We now prove the equilibrium existence in a symmetric equilibrium where options are priced but no traded, and then we generalize using the implicit function theorem.

\footnotetext{
${ }^{4}$ More precisely, this set is the finite union of hyperplans of $\mathbb{R}^{n}$.
} 
Proposition 10 (Equilibrium existence) We suppose that the condition 11) still holds. In the general case with options, an equilibrium where unemployed agents of all types are excluded from the $L+1$ financial markets while employed agents of all types trade strictly positive quantities of assets and of the $L$ options, exists as soon as the tree size $V$ is not too large and the heterogeneity in $\alpha$ and the aggregate uncertainty remain limited.

As there is a finite number of equations, an equilibrium with incomplete markets for both the aggregate and idiosyncratic risk can be easily computed, as is done in the next section. The other interest of this general case is that it allows to identify the conditions under which options are traded in positive volume in our equilibrium. The first condition is that agents face binding credit constraints and want to self-insure. In case of an unconstrained economy, security volumes are undetermined at the equilibrium, and there is no particular role for options. The second condition is that agents face in addition to their idiosyncratic risk, an aggregate uncertainty, such that the market for the aggregate risk remains incomplete, at least before the option introduction. Without uncertainty, the market is complete with a single asset and the redundant option is priced by portfolio replication as in the seminal paper of Black and Scholes (1973). There is no role for option volumes in this framework. The last condition is that agents have heterogeneous risk appetites, such that they are willing to exchange insurance with each other. Otherwise, agents hold similar portfolios and there is no role for options, which only transfer risk from one agent to another. Heterogeneity in risk appetite can come either from the different probability to fall into unemployment, as in the previous section, or from the different productivity levels.

The following proposition summarizes this result.

Proposition 11 Options volumes are undetermined in our economy when one of the following conditions hold:

(i) Unemployed agents do not face binding credit constraints.

(ii) The aggregate risk vanishes $y_{k}=y_{h}$, for all $k, h=1, \ldots, n$.

(iii) The idiosyncratic risk is null $\alpha^{i}=0, i=1, \ldots, I$

(iv) There is no heterogeneity among agents $\alpha_{i}=\alpha_{j}$, and $w_{i}=w_{j}$, for $i, j=1, \ldots, I$.

\section{Numerical example}

As a simple numerical example without any quantitative ambitions, we calibrate the model in the case of two types of agents with an aggregate risk taking two values and with a time varying 
idiosyncratic risk. As before, agents of type 1 have a higher unemployment risk than agents of type 2. This illustrative calibration exercise is done to show that the model can roughly match the difference in the price of the underlying asset, in the price of the option and in the volume of options in the business cycle.

We start to define good and bad aggregate states. We use Hamilton procedure to estimate a two state Markov chain on the S\&P 500 at a monthly $\left.\right|_{5} ^{5}$ frequency during the period 1999-M1 to 2008-M12 for which we could obtain monthly data on options on the S\&P 500. We obtain two regimes, with the four transition probabilities. The transition matrix between the two aggregate shocks is the following:

$$
T=\left[\begin{array}{ll}
0.9452 & 0.0548 \\
0.2381 & 0.7619
\end{array}\right]
$$

In other words, the probability to stay in good times is 0.95 when we are in good times, whereas the probability to stay in bad times when we are in bad times is 0.76 . The average duration of bad times is thus smaller than the duration of good times what is the standard outcome in this type of estimation (Hamilton (1994)).

We can compute the average level $\widetilde{P}$ of the S\&P 500, the dividend $\widetilde{y}$ of the S\&P 500, the average price $\widetilde{Q}$ of the options on the S\&P 500, and the average traded open interest $\widetilde{s}$ (after removing a deterministic trend), in the good state of the world and in the bad one. We denote state $B$ as the bad state (low price and low dividend for the S\&P 500). As a consequence, $\widetilde{P}_{B}$ is the deviation from trend of S\&P 500 in the bad state of the world. The tilde above the variables indicates that these values are estimated from the data. We summarize the data by providing the difference of the values between the bad and the good state of the world. The results are the following ones:

$$
\begin{array}{clll}
\widetilde{P}_{G} / \widetilde{P}_{B} & \widetilde{y}_{G} / \widetilde{y}_{B} & \widetilde{Q}_{G} / \widetilde{Q}_{B} & \widetilde{s}_{G} / \widetilde{s}_{B} \\
\hline \hline 1.26 & 1.18 & 1.19 & 0.95
\end{array}
$$

The previous Table shows that the dividend and price of the S\&P 500 are both higher in the good state of the world, what defines a good state of the world in our economy. The price of the option is higher in the good state of the world and the volume of option traded is higher in the bad state of the world. This could be expected as the volatility of asset prices is higher during bad times.

We now provide a simple calibration to show that the model is able to reproduce the values given in the previous table. To simplify notations, we denote by the upperscript $H$ the values concerning agents with a high level of idiosyncratic risk. We first use the same transition matrix

\footnotetext{
${ }^{5}$ More specifically, we consider deviations from a deterministic trend on the S\&P 500. Data are taken from Shiller website.
} 
between aggregate state than the one found in the data : $\pi=T$. Second, we assume that the utility is CRRA, such the marginal utility is $u^{\prime}(c)=c^{-\sigma}$. Preference parameters as well as the asset size and the domestic production are calibrated as follows.

\begin{tabular}{cccc}
$\sigma$ & $\beta$ & $\delta$ & $V$ \\
\hline \hline 2 & 0.96 & .5 & .1
\end{tabular}

$\sigma$ is the relative risk aversion. $\beta$ is the discount factor $\delta$ is the income of unemployed and $V$ is the volume of the risky asset which has to be small for the equilibrium to exist. The values of the time-varying parameters is the following

\begin{tabular}{llllll} 
& $y$ & $\alpha^{H}(\%)$ & $\alpha^{L}(\%)$ & $w$ & $K$ \\
\hline \hline State $B$ & 0.0107 & 0.63 & 3.74 & 1 & 0.9 \\
State $G$ & 0.0126 & 0.73 & 3.79 & 1 & 1.16
\end{tabular}

The values of the dividend process are actually taken from the empirical estimation, the values of the probabilities to fall into unemployment for both types of agents $\alpha^{H}$ and $\alpha^{L}$ are calibrated to match the data. The value of the probability to get out of unemployment is set to the values necessary to insure that the number of each type of employed agent: 6 is 1 . The wage of each worker when employed is assumed to be constant and equal to 1. Option strikes are arbitrarily chosen between the two prices of the risky asset in both state of the world, as we do not have the data on the traded volume for each strike. One can check that assumptions A-D of the paper are satisfied in this economy. The result of the model is given in the next table, where the data values are recalled :

\begin{tabular}{lllll} 
& $P_{G} / P_{B}$ & $y_{G} / y_{B}$ & $Q_{G} / Q_{B}$ & $s_{G}^{H} / s_{B}^{H}$ \\
\hline \hline Model & 1.26 & 1.18 & 1.31 & 0.95 \\
Data & 1.26 & 1.18 & 1.19 & 0.95
\end{tabular}

The Table shows that the model is roughly able to match the data, although the variance of the price of the option is a little bit too high in this example. To obtain the intuition about the equilibrium allocation, the next table provides equilibrium portfolio.

\begin{tabular}{llll} 
& $s^{H}$ & $x^{H}$ & $x^{L}$ \\
\hline \hline State $B$ & -0.0126 & 0.0648 & 0.0352 \\
State $G$ & -0.0119 & 0.0513 & 0.0487
\end{tabular}

First, high risk agents sell the call option to low risk agents $\left(s^{L}=-s^{H}\right)$. As a consequence, the low risk agents sell insurance about the aggregate risk. The high risk agents hold a higher amount

\footnotetext{
${ }^{6}$ See Challe, Le Grand and Ragot (2007) for a description of the formula to get this result. As the probability to get out of unemployment does not affect equilibrium allocation and prices this is just a normalization assumption.
} 
of the risky asset in both state of the world compared to the low risk agents. The high risk agents hold a higher amount in the bad state of the world because they want more asset in the bad state of the world to self-insure. All these results are illustration of the mechanisms described in the previous sections.

\section{Conclusion}

We present an equilibrium where infinitely-lived agents face both aggregate risk and uninsurable idiosyncratic risk. Due to heterogeneity in the exposure to idiosyncratic risk, agents assess differently the aggregate risk. This creates opportunities to exchange risk through derivative trading. The uninsurable risk is modeled as an unemployment risk, what allows us to obtain a tractable framework, as in Scheinkman and Weiss (1986) or Lagos and Wright (2005) among others.

This framework allows us first to obtain simple pricing equations for derivative assets, when they are not priced by replication. Second, we derive implications for the volume of derivatives exchanged in the business cycle, which is consistent with the data. Third, we analyze the welfare implication of the introduction of derivatives and show that these ones are not Pareto improving. We conclude with a simple numerical example. A route for future research could be to find simplifying assumption to confront more directly this theory of derivative trading to the data. 


\section{References}

Anthony, J. H. (1988): "The Interrelation of Stock and Options Market Trading-Volume Data," The Journal of Finance, 43(4), 949-964.

Bhamra, H. S., and R. Uppal (2009): "The Effect of Introducing a Non-Redundant Derivative on the Volatility of Stock-Market Returns When Agents Differ in Risk Aversion," The Review of Financial Studies, 22(6), 2303-2330.

Biais, B. R., And P. Hillion (1994): "Insider and Liquidity Trading in Stock and Options Markets," The Review of Financial Studies, 7(4), 743-780.

Black, F. S., And M. S. Scholes (1973): "The Pricing of Options and Corporate Liabilities," Journal of Political Economy, 81(3), 637Ü-654.

Bowman, D., and J. Faust (1997): "Options, Sunspots and the Creation of Uncertainty," Journal of Political Economy, 105(5), 957-975.

Buraschi, A., And A. Jiltsov (2006): "Model Uncertainty and Option Markets with Heterogeneous Beliefs," The Journal of Finance, 61(6), 2841-2897.

Cass, D., and A. Citanna (1998): "Pareto Improving Financial Innovation in Incomplete Markets," Economic Theory, 11(1), 467-494.

Challe, E., F. LeGrand, and X. Ragot (2007): "Incomplete Markets, Liquidation Risk and the Term Structure of Interest Rates," PSE WP-2007-49.

Constantinides, G. M., and D. Duffie (1996): "Asset Pricing with Heterogeneous Consumers," Journal of Political Economy, 104(2), 219-240.

Donders, M. W. M., R. R. P. Kouwenberg, and T. A. C. F. Vorst (2000): "Options and Earnings Announcements: An Empirical Study of Volatility, Trading Volume, Open Interest and Liquidity," European Financial Management, 6(2).

Easley, D., M. O’Hara, and P. S. Srinivas (1998): "Option Volume and Stock Prices: Evidence on Where Informed Traders Trade," The Journal of Finance, 45(1), 191-220.

ELuL, R. (1995): "Welfare Effects of Financial Innovation in Incomplete Markets Economies with Several Consumption Goods," Journal of Economic Theory, 65(1), 43-78.

Franke, G., R. C. Stapleton, and M. G. Subrahmanyam (1998): "Who Buys and Who Sells Options: The Role of Options in an Economy with Background Risk," Journal of Economic Theory, 82(1), 89-109.

Hamilton, J. D. (1994): Time Series Analysis. Princeton University Press.

Huggett, M. (1993): "The Risk Free Rate in Heterogeneous-Agent Incomplete-Insurance Economies," Journal of Economic Dynamics and Control, 17(5-6), 953-969.

Karpoff, J. M. (1987): "The Relation Between Price Changes and Trading Volume: A Survey 
," Journal of Financial and Quantitative Analysis, 22(1), 109-126.

Krussel, P., T. Mukoyama, and A. A. J. Smith (2008): "Asset Prices in a Huggett Economy," mimeo.

Lagos, R., and R. Wright (2005): "A Unified Framework for Monetary Theory and Policy Analysis," Journal of Political Economy, 113(3), 463-484.

Ni, S., J. Pan, and A. M. Poteshman (2008): "Volatility Information Trading in the Option Market," The Journal of Finance, 63(3), 1059-1091.

Pan, J., And A. M. Poteshman (2006): "The Information in Option Volume for Future Stock Prices," Review of Financial Studies, 19(3), 871-908.

Scheinkman, J., And L. Weiss (1986): "Borrowing Constraints and Aggregate Economic Activity," Econometrica, 54(1), 23-45.

Stephan, J. A., and R. E. Whaley (1990): "Intraday Price Change and Trading Volume Relations in the Stock and Stock Option Markets," The Journal of Finance, 45(1), 191-220. 


\section{Appendix}

\section{A Proof of Proposition 1}

A solution to formally prove the equilibrium existence is to notice that the equilibrium exists when the asset tree is null and when there is no heterogeneity and no aggregate risk (Section A.1), then to prove using the implicit function theorem that endogenous variables of the economy are continuous with respect to $V$, to dividends $y_{k}(k=B, G)$ and to probabilities $\alpha^{i}, i=1,2$ (Section A.2.

\section{A.1 Symmetric economy}

Zero volume. We consider a symmetric economy without aggregate risk: $y_{B}=y_{G}=y>0$ for all $i, k$. The market equilibrium implies $x=\frac{V}{\eta^{1}+\eta^{2}}$. In the case of zero volume, the asset price $P$ solves $P=\beta\left(1+\alpha\left(u^{\prime}(\delta)-1\right)\right)(P+y)$, which implies:

$$
P=\frac{\beta\left(1+\alpha\left(u^{\prime}(\delta)-1\right)\right)}{1-\beta\left(1+\alpha\left(u^{\prime}(\delta)-1\right)\right)} y>0
$$

The condition for the equilibrium price to be positive is $\beta\left(1+\alpha\left(u^{\prime}(\delta)-1\right)\right)<1$.

For unemployed agents not to take part into the financial market, we need to have:

$$
P u^{\prime}(\delta)>\beta\left(\rho+(1-\rho) u^{\prime}(\delta)\right)(P+y)
$$

Substituting the price $P$ by its expression, this condition is equivalent to

$$
\alpha\left(u^{\prime}(\delta)-1\right)\left(u^{\prime}(\delta)+\frac{\rho}{\alpha}\right)>0
$$

For this condition to hold, we need that $u^{\prime}(\delta)>1$ (Assumption B. It is easy to see that both conditions are fulfilled when condition [11] is fulfilled.

Positive supply. When the asset is in positive supply, $V>0$, the price satisfies the relationship:

$$
P-\beta\left(1+\alpha\left(u^{\prime}\left(\delta+(P+y) \frac{V}{\eta^{1}+\eta^{2}}\right)-1\right)\right)(P+y)=0
$$

The condition for this equilibrium to exist (and exclude unemployed from financial markets) is:

$$
P u^{\prime}\left(\delta+(P+y) \frac{V}{\eta^{1}+\eta^{2}}\right)>\beta\left(\rho+(1-\rho) u^{\prime}(\delta)\right)(P+y)
$$

The equality 27 defines the price as an implicit function of the volume $V$. To prove the existence of the equilibrium with small volumes, one has first to prove the continuity of $P$ as a function of $V$ around the point $V=0$. To do so, one can rewrite the equation 27) as $G(P, V)=0$. It is obvious that $G$ is continuous and derivable in $V$. The derivative $G_{P}(P, 0)$

$$
G_{P}(P, 0)=1-\beta\left(1+\alpha\left(u^{\prime}(\delta)-1\right)\right)>0
$$


By the implicit function theorem, the equality 27] defines $P$ as a continuous function of $V$ around $V=0$. As $P$ is continuous in $V$, and as condition 28 is fulfilled in $V=0$, there exists a neighborhood $W_{1}(0) \in \mathbb{R}^{+}$such that condition 28 is fulfilled. Define $V^{*}>0$ a point of $W_{1}(0)$ and $P^{*}$ the price of the asset for this volume. At this equilibrium, the quantity of asset held by each agent is $x^{*}=\frac{V^{*}}{\eta^{1}+\eta^{2}}>0$.

\section{A.2 General case}

We define $X=\left(y_{B}, y_{G}, V, \alpha^{1}, \alpha^{2}\right) \in\left(\mathbb{R}^{+}\right)^{5}$ the vector of parameters and $Z=\left(P_{B}, P_{G}, x_{B}^{1}, x_{G}^{1}, x_{B}^{2}, x_{G}^{2}\right) \in\left(\mathbb{R}^{+}\right)^{6}$ the vector of endogenous variables.

We define the vector $F$ of length 6 of equations as $(i=1,2$ and $k=1,2)$ :

$$
\begin{aligned}
& F_{1}(Z, X)=P_{B}-\beta \sum_{j=B, G} \pi_{B, j}\left(1+\alpha^{1} u^{\prime}\left(\delta+\left(P_{j}+y_{j}\right) x_{B}^{1}\right)\right)\left(P_{j}+y_{j}\right) \\
& F_{2}(Z, X)=P_{G}-\beta \sum_{j=B, G} \pi_{G, j}\left(1+\alpha^{1} u^{\prime}\left(\delta+\left(P_{j}+y_{j}\right) x_{G}^{1}\right)\right)\left(P_{j}+y_{j}\right) \\
& F_{3}(Z, X)=P_{B}-\beta \sum_{j=B, G} \pi_{B, j}\left(1+\alpha^{2} u^{\prime}\left(\delta+\left(P_{j}+y_{j}\right) x_{B}^{2}\right)\right)\left(P_{j}+y_{j}\right) \\
& F_{4}(Z, X)=P_{G}-\beta \sum_{j=B, G} \pi_{G, j}\left(1+\alpha^{2} u^{\prime}\left(\delta+\left(P_{j}+y_{j}\right) x_{G}^{2}\right)\right)\left(P_{j}+y_{j}\right) \\
& F_{5}(Z, X)=\eta^{1} x_{B}^{1}+\eta^{2} x_{B}^{2}-V \\
& F_{6}(Z, X)=\eta^{1} x_{G}^{1}+\eta^{2} x_{G}^{2}-V
\end{aligned}
$$

$F$ stacks pricing functions for both agents' types and the market equilibrium. The first 2 equations are the pricing equations of type 1 agents in states $B$ and $G$, the next 2 equations concern agents of type 2 , and so on and so forth. The last 2 equations is the set of market equilibria.

For a given set of parameters $X$, the equilibrium is defined as:

$$
F(Z, X)=0
$$

- There exists an equilibrium for $X^{*}=\left(y_{B}, y_{G}, V^{*}, \alpha^{1}, \alpha^{2}\right)$, where unemployed do not trade the asset. The equilibrium is defined by $Z^{*}=\left(P^{*}, P^{*}, x^{*}, x^{*}, x^{*}, x^{*}\right)$.

- To prove the equilibrium existence in the vicinity of $X^{\star}$, we show that the Jacobian $\Delta$ of $F$ relative to $Y$ is invertible:

$$
\Delta=\left(\frac{\partial F_{i}}{\partial z_{j}}\left(Z^{*}, X^{*}\right)\right)_{i, j=1, \ldots, 6}
$$


- We derive relative to $y_{l}, l=B, G(i=1,2$ and $k=1,2)$. For sake of simplicity, we suppose that $k=1$ is similar to $k=B$ and $k=2$ is similar to $k=G$ :

$$
\begin{aligned}
\frac{\partial F_{2(i-1)+k}}{\partial P_{l}} & =1_{k=l}-\beta \pi_{k, l}\left(1+\alpha^{i}\left(u^{\prime}\left(\left(P_{l}+y_{l}\right) x_{k}^{i}+\delta\right)-1\right)\right) \\
& -\beta \pi_{k, l} \alpha^{i} x_{k}^{i} u^{\prime \prime}\left(\left(P_{l}+y_{l}\right) x_{k}^{i}+\delta\right)\left(P_{l}+y_{l}\right) \\
\frac{\partial F_{4+k}}{\partial P_{l}} & =0
\end{aligned}
$$

- Second, for $i, i^{\prime}=1,2, k, h=1,2$ (or similarly $\left.B, G\right)(k \neq h)$ :

$$
\begin{array}{rlrl}
\frac{\partial F_{2(i-1)+k}}{\partial x_{h}^{i^{\prime}}} & =0 \\
\frac{\partial F_{2(i-1)+k}}{\partial x_{k}^{i}} & =-\beta \alpha^{i} \quad \sum_{j=B, G} \pi_{k, j} u^{\prime \prime}\left(\left(P_{j}+y_{j}\right) x_{k}^{i}+\delta\right)\left(P_{j}+y_{j}\right)^{2} \\
\frac{\partial F_{4+h}}{\partial x_{k}^{i}} & =0 & \frac{\partial F_{4+k}}{\partial x_{k}^{i}}=1
\end{array}
$$

- In the vicinity of the symmetric certain equilibrium, the Jabobian matrix $\Delta$ has this simple shape:

$$
\Delta=\left[\begin{array}{ccc}
A & C & 0_{2 \times 2} \\
A & 0_{2 \times 2} & C \\
0_{2 \times 2} & 1_{2 \times 2} & 1_{2 \times 2}
\end{array}\right]
$$

$C=-\beta \alpha u^{\prime \prime}\left(\left(P^{*}+y\right) x+\delta\right)\left(P^{*}+y\right)^{2} \operatorname{diag}(1,1)>0$ is a $2 \times 2$ diagonal matrix. $A$ is a $2 \times 2$ matrix such that $A_{k, j}=1_{k=j}-\beta \pi_{k, j} M$ with:

$$
M=1+\alpha\left(u^{\prime}\left(\left(P^{*}+y\right) x^{*}+\delta\right)-1+x^{*} u^{\prime \prime}\left(\left(P^{*}+y\right) x+\delta\right)\left(P^{*}+y\right)\right)
$$

The matrix $A$ can be written as $A=I_{2}-\beta M T\left(I_{2}\right.$ is the $2 \times 2$ identity matrix and $\left.T=\left(\pi_{k, l}\right)_{k, l=B, G}\right)$ and is invertible. Indeed, Assumption 11 implies that $0<\beta M<1$. It is easy to check that the matrix $A A=w\left(\sum_{k=0}^{\infty}(\varphi T)^{k}\right)$ exists and verifies $A A A=I_{n}$ ( $I_{n}$ is the identity matrix).

- We now prove that the columns of $\Delta$ are independent of each other and that $\Delta$ is invertible. Let $X=\left(X_{0}, X_{1}, X_{2}\right) \in(\mathbb{R})^{6} . X \in \operatorname{ker} \Delta$ implies:

$$
\begin{aligned}
j=1,2, A X_{0}+C X_{j} & =0_{2} \\
X_{1}+X_{2} & =0_{2}
\end{aligned}
$$

Summing the 2 first equalities over $i$ provides $2 A X_{0}+C\left(X_{1}+X_{2}\right)=2 A X_{0}=0_{2}$. Since $A$ is invertible, we obtain $X_{0}=0_{2}$. We deduce that for $i=1,2, C X_{i}=0_{n}$ and that $X_{i}=0_{2}(C$ is diagonal with strictly positive elements). We prove thus that ker $\Delta=\left\{0_{2}\right\}$ and that $\Delta$ is invertible.

As the Jacobian of $F$ with respect to $Y$ is invertible at the point $\left(X^{*}, Y^{*}\right)$, the implicit function theorem proves that there exists a function $G$ such that $Y=G(X)$, for $X$ close to $X^{*}$. In consequence, our equilibrium in the vicinity of $\left(X^{*}, Y^{*}\right)$. 


\section{B Proof of Proposition 2}

The equilibrium is defined with the following equations $(k=G, B$ and $i=1,2)$ :

$$
\begin{array}{ll} 
& P_{k}=\beta \sum_{j=B, G} \pi_{k, j}\left(1+\alpha^{i}\left(u^{\prime}\left(\delta+\left(P_{j}+y_{j}\right) x_{k}^{i}\right)-1\right)\right)\left(P_{j}+y_{j}\right) \\
\text { with: } & \sum_{j=B, G} \pi_{k, j}\left(P_{j}+y_{j}\right)+\alpha^{1} \pi_{k, j}\left(u^{\prime}\left(\delta+\left(P_{j}+y_{j}\right) x_{k}^{1}\right)-1\right)\left(P_{j}+y_{j}\right) \\
& =\sum_{j=B, G} \pi_{k, j}\left(P_{j}+y_{j}\right)+\alpha^{2} \pi_{k, j}\left(u^{\prime}\left(\delta+\left(P_{j}+y_{j}\right) x_{k}^{2}\right)-1\right)\left(P_{j}+y_{j}\right) \\
\text { and: } \quad & \eta^{1} x_{k}^{1}+\eta^{2} x_{k}^{2}=V
\end{array}
$$

We study the evolution of prices and quantities around the certain equilibrium $y_{G}=y_{B}=y$, where the asset price is constant to $P$ and the agents' asset holdings are denoted respectively $x^{1}$ and $x^{2}$. The equilibrium variables are defined as:

$$
\begin{aligned}
& P=\beta\left(1+\alpha^{i}\left(u^{\prime}\left(\delta+(P+y) x^{i}\right)-1\right)\right)(P+y) \\
& \text { with: } \alpha^{1}\left(u^{\prime}\left(\delta+(P+y) x^{1}\right)-1\right)=\alpha^{2}\left(u^{\prime}\left(\delta+(P+y) x^{2}\right)-1\right) \\
& \text { and: } \eta^{1} x^{1}+\eta^{2} x^{2}=V
\end{aligned}
$$

\section{B.1 Quantities}

We derive the equation 30 linking $x_{k}^{1}$ and $x_{k}^{2}$ relative to $y_{l}(l=B, G)$ :

$$
\begin{aligned}
& \sum_{j=B, G} \pi_{k, j}\left(1+\alpha^{1}\left(u^{\prime}\left(\delta+\left(P_{j}+y_{j}\right) x_{k}^{1}\right)-1\right)\right)\left(\frac{\partial P_{j}}{\partial y_{l}}+1_{j=l}\right) \\
& \quad+\sum_{j=B, G} \pi_{k, j} \alpha^{1} u^{\prime \prime}\left(\delta+\left(P_{j}+y_{j}\right) x_{k}^{1}\right)\left(P_{j}+y_{j}\right)\left(x_{k}^{1}\left(\frac{\partial P_{j}}{\partial y_{l}}+1_{j=l}\right)+\left(P_{j}+y_{j}\right) \frac{\partial x_{k}^{1}}{\partial y_{l}}\right) \\
& =\sum_{j=B, G} \pi_{k, j}\left(1+\alpha^{2}\left(u^{\prime}\left(\delta+\left(P_{j}+y_{j}\right) x_{k}^{2}\right)-1\right)\right)\left(\frac{\partial P_{j}}{\partial y_{l}}+1_{j=l}\right) \\
& \quad+\sum_{j=B, G} \pi_{k, j} \alpha^{2} u^{\prime \prime}\left(\delta+\left(P_{j}+y_{j}\right) x_{k}^{2}\right)\left(P_{j}+y_{j}\right)\left(x_{k}^{2}\left(\frac{\partial P_{j}}{\partial y_{l}}+1_{j=l}\right)+\left(P_{j}+y_{j}\right) \frac{\partial x_{k}^{2}}{\partial y_{l}}\right)
\end{aligned}
$$

Close to the certain equilibrium, the previous equation simplifies into:

$$
\begin{aligned}
& \left(1+\alpha^{1}\left(u^{\prime}\left(\delta+(P+y) x^{1}\right)-1\right)\right) \sum_{j=B, G} \pi_{k, j}\left(\frac{\partial P_{j}}{\partial y_{l}}+1_{j=l}\right) \\
& +\alpha^{1} u^{\prime \prime}\left(\delta+(P+y) x^{1}\right)(P+y) \sum_{j=B, G} \pi_{k, j}\left(x^{1}\left(\frac{\partial P_{j}}{\partial y_{l}}+1_{j=l}\right)+(P+y) \frac{\partial x_{k}^{1}}{\partial y_{l}}\right) \\
& =\left(1+\alpha^{2}\left(u^{\prime}\left(\delta+(P+y) x^{2}\right)-1\right)\right) \sum_{j=B, G} \pi_{k, j}\left(\frac{\partial P_{j}}{\partial y_{l}}+1_{j=l}\right) \\
& +\alpha^{2} u^{\prime \prime}\left(\delta+(P+y) x^{2}\right)(P+y) \sum_{j=B, G} \pi_{k, j}\left(x^{2}\left(\frac{\partial P_{j}}{\partial y_{l}}+1_{j=l}\right)+(P+y) \frac{\partial x_{k}^{2}}{\partial y_{l}}\right)
\end{aligned}
$$


Using the equality 33 , we deduce:

$$
\begin{aligned}
& \alpha^{1} u^{\prime \prime}\left(\delta+(P+y) x^{1}\right) \sum_{j=B, G} \pi_{k, j}\left(x^{1}\left(\frac{\partial P_{j}}{\partial y_{l}}+1_{j=l}\right)+(P+y) \frac{\partial x_{k}^{1}}{\partial y_{l}}\right) \\
& =\alpha^{2} u^{\prime \prime}\left(\delta+(P+y) x^{2}\right) \sum_{j=B, G} \pi_{k, j}\left(x^{2}\left(\frac{\partial P_{j}}{\partial y_{l}}+1_{j=l}\right)+(P+y) \frac{\partial x_{k}^{2}}{\partial y_{l}}\right)
\end{aligned}
$$

We derive the equation 31 relative to $y_{l}$ and deduce $\frac{\partial x_{k}^{2}}{\partial y_{l}}=-\frac{\eta^{1}}{\eta^{2}} \frac{\partial x_{k}^{1}}{\partial y_{l}}$. The previous equality simplifies into:

$$
\begin{aligned}
& \eta^{1}(P+y) \frac{\partial x_{k}^{1}}{\partial y_{l}}\left(\frac{\alpha^{1}}{\eta^{1}} u^{\prime \prime}\left(\delta+(P+y) x^{1}\right)+\frac{\alpha^{2}}{\eta^{2}} u^{\prime \prime}\left(\delta+(P+y) x^{2}\right)\right) \\
& =\left(\alpha^{2} x^{2} u^{\prime \prime}\left(\delta+(P+y) x^{2}\right)-\alpha^{1} x^{1} u^{\prime \prime}\left(\delta+(P+y) x^{1}\right)\right) \sum_{j=B, G} \pi_{k, j}\left(\frac{\partial P_{j}}{\partial y_{l}}+1_{j=l}\right)
\end{aligned}
$$

We deduce finally:

$$
\begin{aligned}
\eta^{1}(P+y) \frac{\partial x_{k}^{1}}{\partial y_{l}} & =\frac{\alpha^{2} x^{2} u^{\prime \prime}\left(\delta+(P+y) x^{2}\right)-\alpha^{1} x^{1} u^{\prime \prime}\left(\delta+(P+y) x^{1}\right)}{\frac{\alpha^{1}}{\eta^{1}} u^{\prime \prime}\left(\delta+(P+y) x^{1}\right)+\frac{\alpha^{2}}{\eta^{2}} u^{\prime \prime}\left(\delta+(P+y) x^{2}\right)} \sum_{j=B, G} \pi_{k, j}\left(\frac{\partial P_{j}}{\partial y_{l}}+1_{j=l}\right) \\
& =\frac{\alpha^{2} x^{2} \frac{u^{\prime \prime}\left(\delta+(P+y) x^{2}\right)}{\alpha^{2}\left(u^{\prime \prime}\left(\delta+(P+y) x^{2}\right)-1\right)}-\alpha^{1} x^{1} \frac{u^{\prime \prime}\left(\delta+(P+y) x^{1}\right)}{\alpha^{1}\left(u^{\prime \prime}\left(\delta+(P+y) x^{1}\right)-1\right)}}{\frac{\alpha^{1}}{\eta^{1}} \frac{u^{\prime \prime}\left(\delta+(P+y) x^{1}\right)}{\alpha^{1}\left(u^{\prime \prime}\left(\delta+(P+y) x^{1}\right)-1\right)}+\frac{\alpha^{2}}{\eta^{2}} \frac{u^{\prime \prime}\left(\delta+(P+y) x^{2}\right)}{\alpha^{2}\left(u^{\prime \prime}\left(\delta+(P+y) x^{2}\right)-1\right)}} \sum_{j=B, G} \pi_{k, j}\left(\frac{\partial P_{j}}{\partial y_{l}}+1_{j=l}\right)
\end{aligned}
$$

We have divided the numerator and the denominator by $\alpha^{1}\left(u^{\prime \prime}\left(\delta+(P+y) x^{1}\right)-1\right)=\alpha^{2}\left(u^{\prime \prime}\left(\delta+(P+y) x^{2}\right)-\right.$ 1). After simplification, we obtain:

$$
\begin{aligned}
\eta^{1}(P+y) \frac{\partial x_{k}^{1}}{\partial y_{l}}=-\eta^{2}(P+y) \frac{\partial x_{k}^{2}}{\partial y_{l}} & =-\kappa \sum_{j=B, G} \pi_{k, j}\left(\frac{\partial P_{j}}{\partial y_{l}}+1_{j=l}\right) \\
\kappa & =\frac{-x^{1} \frac{u^{\prime \prime}\left(\delta+(P+y) x^{1}\right)}{u^{\prime}\left(\delta+(P+y) x^{1}\right)-1}+x^{2} \frac{u^{\prime \prime}\left(\delta+(P+y) x^{2}\right)}{u^{\prime}\left(\delta+(P+y) x^{2}\right)-1}}{-\frac{1}{\eta^{1}} \frac{u^{\prime \prime}\left(\delta+(P+y) x^{1}\right)}{u^{\prime}\left(\delta+(P+y) x^{1}\right)-1}-\frac{1}{\eta^{2}} \frac{u^{\prime \prime}\left(\delta+(P+y) x^{2}\right)}{u^{\prime}\left(\delta+(P+y) x^{2}\right)-1}}>0
\end{aligned}
$$

$\kappa$ is supposed to be strictly positive (Assumption D. We prove that it holds for standard utility classes. More precisely, we show that $x \mapsto-x \frac{u^{\prime \prime}(\delta+x)}{u^{\prime}(\delta+x)-1}$ is increasing.

Computation of $\kappa$ for CARA. We suppose that $u(c)=-\lambda \exp (-\sigma c)$.

$$
-x \frac{u^{\prime \prime}(\delta+x)}{u^{\prime}(\delta+x)-1}=x \frac{\sigma^{2} \lambda \exp (-\sigma(\delta+x))}{\sigma \lambda \exp (-\sigma(\delta+x))-1}=\frac{x \sigma}{1-\exp (\sigma(\delta+x)) /(\sigma \lambda)}
$$

The derivative relative to $x$ is:

$$
\begin{aligned}
\frac{1}{1-\exp (\sigma(\delta+x)) /(\sigma \lambda)}+\frac{x \exp (\sigma(\delta+x)) / \lambda}{(1-\exp (\sigma(\delta+x)) /(\sigma \lambda))^{2}} & =\frac{1-\exp (\sigma(\delta+x)) /(\sigma \lambda)+x \exp (\sigma(\delta+x)) / \lambda}{(1-\exp (\sigma(\delta+x)) /(\sigma \lambda))^{2}} \\
& >\frac{x \exp (\sigma(\delta+x)) / \lambda}{(1-\exp (\sigma(\delta+x)) /(\sigma \lambda))^{2}}>0
\end{aligned}
$$


Computation of $\kappa$ for CRRA. We suppose that $u^{\prime}(c)=\lambda c^{-\sigma}$.

$$
-x \frac{u^{\prime \prime}(\delta+x)}{u^{\prime}(\delta+x)-1}=x \frac{\sigma \lambda(\delta+x)^{-\sigma-1}}{\lambda(\delta+x)^{-\sigma}-1}=\frac{x}{x+\delta} \frac{\sigma}{1-(\delta+x)^{\sigma} / \lambda}
$$

The derivative relative to $x$ is:

$$
\begin{aligned}
& \frac{\delta}{(x+\delta)^{2}} \frac{1}{1-(\delta+x)^{\sigma} / \lambda}+\frac{x}{x+\delta} \frac{\sigma(\delta+x)^{\sigma-1} / \lambda}{\left(1-(\delta+x)^{\sigma} / \lambda\right)^{2}} \\
& =\frac{1}{x+\delta} \frac{1}{1-(\delta+x)^{\sigma} / \lambda}\left(\frac{\delta}{x+\delta}+x \frac{\sigma(\delta+x)^{\sigma-1} / \lambda}{1-(\delta+x)^{\sigma} / \lambda}\right) \\
& =\frac{1}{x+\delta} \frac{1}{1-(\delta+x)^{\sigma} / \lambda}\left(\frac{\delta}{x+\delta}+x \frac{\sigma(\delta+x)^{\sigma-1} / \lambda}{1-(\delta+x)^{\sigma} / \lambda}\right)>0
\end{aligned}
$$

Computation of $\kappa$ for quadratic utility functions. We suppose that $u^{\prime}(c)=a-b c$.

$$
-x \frac{u^{\prime \prime}(\delta+x)}{u^{\prime}(\delta+x)-1}=x \frac{b}{a-b(\delta+x)-1}
$$

The derivative relative to $x$ is:

$$
b \frac{a-b(\delta+x)-1+b x}{(a-b(\delta+x)-1)^{2}}=b \frac{a-b \delta-1}{(a-b(\delta+x)-1)^{2}}>0
$$

\section{B.2 Prices}

We derive the equation 29 relative to $y_{l}, l=B, G$ in the vicinity of the certain equilibrium:

$$
\begin{aligned}
\frac{\partial P_{k}}{\partial y_{l}} & =\beta\left(1+\alpha^{i}\left(u^{\prime}\left(\delta+(P+y) x^{i}\right)-1\right)\right) \sum_{j=B, G} \pi_{k, j}\left(\frac{\partial P_{j}}{\partial y_{l}}+1_{j=l}\right) \\
& +\beta \alpha^{i} u^{\prime \prime}\left(\delta+(P+y) x^{i}\right)(P+y) \sum_{j=B, G} \pi_{k, j}\left(x^{i}\left(\frac{\partial P_{j}}{\partial y_{l}}+1_{j=l}\right)+(P+y) \frac{\partial x_{k}^{i}}{\partial y_{l}}\right) \\
& =\beta\left(1+\alpha^{i}\left(u^{\prime}\left(\delta+(P+y) x^{i}\right)-1\right)\left(1+\left(x^{i}+(-1)^{i} \frac{\kappa}{\eta^{i}}\right) \frac{u^{\prime \prime}\left(\delta+(P+y) x^{i}\right)}{u^{\prime}\left(\delta+(P+y) x^{i}\right)-1}\right) \sum_{j=B, G} \pi_{k, j}\left(\frac{\partial P_{j}}{\partial y_{l}}+1_{j=l}\right)\right. \\
& =\beta \widehat{M} \sum_{j=B, G} \pi_{k, j}\left(\frac{\partial P_{j}}{\partial y_{l}}+1_{j=l}\right) \\
\widehat{M} & =1+\alpha^{i}\left(u^{\prime}\left(\delta+(P+y) x^{i}\right)-1\right)\left(1-\frac{V}{\eta^{1} \eta^{2}} \frac{u^{\prime \prime}\left(\delta+(P+y) x^{1}\right)}{u^{\prime}\left(\delta+(P+y) x^{1}\right)-1} \frac{u^{\prime \prime}\left(\delta+(P+y) x^{2}\right)}{u^{\prime}\left(\delta+(P+y) x^{2}\right)-1}\right)
\end{aligned}
$$

$\widehat{M}$ is the modified pricing kernel for the valuation of one additional unit of dividend tomorrow.

We deduce:

$$
\left\{\begin{array}{l}
\left(1-\beta \widehat{M} \pi_{G G}\right) \frac{\partial P_{G}}{\partial y_{l}}=\beta \widehat{M}\left(\pi_{G G} 1_{l=G}+\pi_{G B}\left(\frac{\partial P_{B}}{\partial y_{l}}+1_{l=B}\right)\right) \\
\left(1-\beta \widehat{M} \pi_{B B}\right) \frac{\partial P_{B}}{\partial y_{l}}=\beta \widehat{M}\left(\pi_{B G}\left(\frac{\partial P_{G}}{\partial y_{l}}+1_{l=G}\right)+\pi_{B B} 1_{l=B}\right)
\end{array}\right.
$$


or

$$
\begin{aligned}
& {\left[\begin{array}{cc}
1-\beta \widehat{M} \pi_{G G} & -\beta \widehat{M} \pi_{G B} \\
-\beta \widehat{M} \pi_{B G} & 1-\beta \widehat{M} \pi_{B B}
\end{array}\right]\left[\begin{array}{c}
\frac{\partial P_{G}}{\partial y_{l}} \\
\frac{\partial P_{B}}{\partial y_{l}}
\end{array}\right]=\beta \widehat{M}\left[\begin{array}{c}
\pi_{G G} 1_{l=G}+\pi_{G B} 1_{l=B} \\
\pi_{B G} 1_{l=G}+\pi_{B B} 1_{l=B}
\end{array}\right]} \\
& \left(\left(1-\beta \widehat{M} \pi_{G G}\right)\left(1-\beta \widehat{M} \pi_{B B}\right)-\beta^{2} \widehat{M}^{2} \pi_{B G} \pi_{G B}\right)\left[\begin{array}{c}
\frac{\partial P_{G}}{\partial y_{l}} \\
\frac{\partial P_{B}}{\partial y_{l}}
\end{array}\right]=\beta \widehat{M}\left[\begin{array}{cc}
1-\beta \widehat{M} \pi_{B B} & \beta \widehat{M} \pi_{G B} \\
\beta \widehat{M} \pi_{B G} & 1-\beta \widehat{M} \pi_{G G}
\end{array}\right]\left[\begin{array}{c}
\pi_{G G} 1_{l=G}+\pi_{G B} 1_{l=B} \\
\pi_{B G} 1_{l=G}+\pi_{B B} 1_{l=B}
\end{array}\right] \\
& \left(1-\beta \widehat{M} \pi_{G G}-\beta \widehat{M} \pi_{B B}-\beta^{2} \widehat{M}^{2}\left(\pi_{B G} \pi_{G B}-\pi_{G G} \pi_{B B}\right)\right)\left[\begin{array}{c}
\frac{\partial P_{G}}{\partial y_{l}} \\
\frac{\partial P_{B}}{\partial y_{l}}
\end{array}\right]=\beta \widehat{M} \\
& {\left[\begin{array}{l}
\left(\pi_{G G}+\beta \widehat{M}\left(\left(1-\pi_{G G}\right)\left(1-\pi_{B B}\right)-\pi_{G G} \pi_{B B}\right)\right) 1_{l=G}+\left(1-\pi_{G G}+\beta \widehat{M}\left(\left(1-\pi_{G G}\right) \pi_{B B}-\left(1-\pi_{G G}\right) \pi_{B B}\right)\right) 1_{l=B} \\
\left(1-\pi_{B B}+\beta \widehat{M}\left(\pi_{G G}\left(1-\pi_{B B}\right)-\pi_{G G}\left(1-\pi_{B B}\right)\right)\right) 1_{l=G}+\left(\pi_{B B}+\beta \widehat{M}\left(\left(1-\pi_{G G}\right)\left(1-\pi_{B B}\right)-\pi_{G G} \pi_{B B}\right)\right) 1_{l=B}
\end{array}\right.} \\
& \left(1-\beta \widehat{M} \pi_{G G}-\beta \widehat{M} \pi_{B B}+\beta^{2} \widehat{M}^{2}\left(\pi_{G G}+\pi_{B B}-1\right)\right)\left[\begin{array}{c}
\frac{\partial P_{G}}{\partial y_{l}} \\
\frac{\partial P_{B}}{\partial y_{l}}
\end{array}\right]=\beta \widehat{M}\left[\begin{array}{c}
\left(\pi_{G G}-\beta \widehat{M}\left(\pi_{G G}+\pi_{B B}-1\right)\right) 1_{l=G}+\left(1-\pi_{G G}\right) 1_{l=B} \\
\left(1-\pi_{B B}\right) 1_{l=G}+\left(\pi_{B B}-\beta \widehat{M}\left(\pi_{G G}+\pi_{B B}-1\right)\right) 1_{l=B}
\end{array}\right.
\end{aligned}
$$

We finally deduce:

$$
\left[\begin{array}{c}
\frac{\partial P_{G}}{\partial y_{l}} \\
\frac{\partial P_{B}}{\partial y_{l}}
\end{array}\right]=\frac{\beta \widehat{M}}{(1-\beta \widehat{M})\left(1-\left(\pi_{G G}+\pi_{B B}-1\right) \beta \widehat{M}\right)}\left[\begin{array}{c}
\left(\pi_{G G}-\beta \widehat{M}\left(\pi_{G G}+\pi_{B B}-1\right)\right) 1_{l=G}+\left(1-\pi_{G G}\right) 1_{l=B} \\
\left(1-\pi_{B B}\right) 1_{l=G}+\left(\pi_{B B}-\beta \widehat{M}\left(\pi_{G G}+\pi_{B B}-1\right)\right) 1_{l=B}
\end{array}\right]>0
$$

and: $\quad \sum_{j=B, G} \pi_{k, j}\left(\frac{\partial P_{j}}{\partial y_{l}}+1_{j=l}\right)=\frac{1}{\beta \widehat{M}} \frac{\partial P_{k}}{\partial y_{l}}$

\section{B.3 Summary}

In the no-option economy, the following relationships hold:

$$
\begin{aligned}
& {\left[\begin{array}{c}
\frac{\partial P_{G}}{\partial y_{l}} \\
\frac{\partial P_{B}}{\partial y_{l}}
\end{array}\right]=} \frac{\beta \widehat{M}}{(1-\beta \widehat{M})\left(1-\left(\pi_{G G}+\pi_{B B}-1\right) \beta \widehat{M}\right)}\left[\begin{array}{c}
\left(\pi_{G G}-\beta \widehat{M}\left(\pi_{G G}+\pi_{B B}-1\right)\right) 1_{l=G}+\left(1-\pi_{G G}\right) 1_{l=B} \\
\left(1-\pi_{B B}\right) 1_{l=G}+\left(\pi_{B B}-\beta \widehat{M}\left(\pi_{G G}+\pi_{B B}-1\right)\right) 1_{l=B}
\end{array}\right] \\
& \eta^{1}(P+y) \frac{\partial x_{k}^{1}}{\partial y_{l}}=-\eta^{2}(P+y) \frac{\partial x_{k}^{2}}{\partial y_{l}}=-\frac{\kappa}{\beta \widehat{M}} \frac{\partial P_{k}}{\partial y_{l}}<0 \\
& \text { with: } \quad \kappa=\frac{-x^{1} \frac{u^{\prime \prime}\left(\delta+(P+y) x^{1}\right)}{u^{\prime \prime}\left(\delta+(P+y) x^{1}\right)-1}+x^{2} \frac{u^{\prime \prime}\left(\delta+(P+y) x^{2}\right)}{u^{\prime \prime}\left(\delta+(P+y) x^{2}\right)-1}}{-\frac{1}{\eta^{1}} \frac{u^{\prime \prime}\left(\delta+(P+y) x^{1}\right)}{u^{\prime \prime}\left(\delta+(P+y) x^{1}\right)-1}-\frac{1}{\eta^{2}} \frac{u^{\prime \prime}\left(\delta+(P+y) x^{2}\right)}{u^{\prime \prime}\left(\delta+(P+y) x^{2}\right)-1}}>0 \\
& \widehat{M}= 1+\alpha^{i}\left(u^{\prime}\left(\delta+(P+y) x^{i}\right)-1\right)\left(1-\frac{V}{\eta^{1} \eta^{2}} \frac{u^{\prime \prime}\left(\delta+(P+y) x^{1}\right)}{u^{\prime}\left(\delta+(P+y) x^{1}\right)-1} \frac{u^{\prime \prime}\left(\delta+(P+y) x^{2}\right)}{u^{\prime}\left(\delta+(P+y) x^{2}\right)-1}\right)
\end{aligned}
$$




\section{B.4 Lemma}

Before going further into the proof, we show the following lemma:

Lemma 12 Let $\Phi: Y \rightarrow \mathbb{R}$ a continuous and derivable function depending on $y_{G}$ and $y_{B}$. We note $V[y]$ (E[y]) the variance (mean) of the dividend process $y$. The impact of a mean preserving spread of dividend expresses as:

$$
\left.\frac{\partial \Phi}{\partial V[y]}\right|_{E[y]=\text { constant }}=\frac{1}{2\left(y_{G}-y_{B}\right)}\left[\frac{2-\pi_{G G}-\pi_{B B}}{1-\pi_{B B}} \frac{\partial \Phi}{\partial y_{G}}-\frac{2-\pi_{G G}-\pi_{B B}}{1-\pi_{G G}} \frac{\partial \Phi}{\partial y_{B}}\right]
$$

Proof. We express $y_{G}$ and $y_{B}$ as a function of $E[y]$ and $V[y]$ :

$$
\begin{aligned}
& y_{G}=E[y]+(1-q) \sqrt{\frac{V[y]}{q(1-q)}} \quad \frac{\partial y_{G}}{\partial V[y]}=(1-q) \frac{1}{2 \sqrt{q(1-q) V[y]}} \\
& y_{B}=E[y]-q \sqrt{\frac{V[y]}{q(1-q)}} \quad \frac{\partial y_{B}}{\partial V[y]}=-q \frac{1}{2 \sqrt{q(1-q) V[y]}} \\
& \text { with: } q=\frac{1-\pi_{B B}}{2-\pi_{G G}-\pi_{B B}}
\end{aligned}
$$

The derivative of $\Phi$ relative to $V[y]$ is:

$$
\begin{aligned}
\frac{\partial \Phi}{\partial V[y]} & =\frac{\partial \Phi}{\partial y_{G}} \frac{\partial y_{G}}{\partial V[y]}+\frac{\partial \Phi}{\partial y_{B}} \frac{\partial y_{B}}{\partial V[y]} \\
& =\frac{1}{2 \sqrt{q(1-q) V[y]}}\left[(1-q) \frac{\partial \Phi}{\partial y_{G}}-q \frac{\partial \Phi}{\partial y_{B}}\right] \\
& =\frac{1}{2 q(1-q)\left(y_{G}-y_{B}\right)}\left[(1-q) \frac{\partial \Phi}{\partial y_{G}}-q \frac{\partial \Phi}{\partial y_{B}}\right] \\
& =\frac{\left(2-\pi_{G G}-\pi_{B B}\right)^{2}}{2\left(1-\pi_{G G}\right)\left(1-\pi_{B B}\right)\left(y_{G}-y_{B}\right)}\left[\left(1-\pi_{G G}\right) \frac{\partial \Phi}{\partial y_{G}}-\left(1-\pi_{B B}\right) \frac{\partial \Phi}{\partial y_{B}}\right]
\end{aligned}
$$

\section{B.5 Back to Proposition 2}

Using previous results, we prove that:

$$
\begin{aligned}
\frac{2\left(y_{G}-y_{B}\right)}{2-\pi_{G G}-\pi_{B B}} \frac{\partial P_{G}}{\partial V[y]} & =\frac{\beta \widehat{M}}{(1-\beta \widehat{M})\left(1-\left(\pi_{G G}+\pi_{B B}-1\right) \beta \widehat{M}\right)}\left(\frac{\pi_{G G}-\beta \widehat{M}\left(\pi_{G G}+\pi_{B B}-1\right)}{1-\pi_{B B}}-1\right) \\
& =\frac{1}{1-\pi_{B B}} \frac{\left(\pi_{G G}+\pi_{B B}-1\right) \beta \widehat{M}}{1-\left(\pi_{G G}+\pi_{B B}-1\right) \beta \widehat{M}}>0 \\
\frac{2\left(y_{G}-y_{B}\right)}{2-\pi_{G G}-\pi_{B B}} \frac{\partial P_{B}}{\partial V[y]} & =\frac{\beta \widehat{M}}{(1-\beta \widehat{M})\left(1-\left(\pi_{G G}+\pi_{B B}-1\right) \beta \widehat{M}\right)}\left(1-\frac{\pi_{B B}-\beta \widehat{M}\left(\pi_{G G}+\pi_{B B}-1\right)}{1-\pi_{G G}}\right) \\
& =-\frac{1}{1-\pi_{G G}} \frac{\left(\pi_{G G}+\pi_{B B}-1\right) \beta \widehat{M}}{1-\left(\pi_{G G}+\pi_{B B}-1\right) \beta \widehat{M}}>0 \\
\eta^{1}(P+y) \frac{\partial x_{k}^{1}}{\partial V[y]} & =-\eta^{2}(P+y) \frac{\partial x_{k}^{2}}{\partial V[y]}=-\frac{\kappa}{\beta \widehat{M}} \frac{\partial P_{k}}{\partial V[y]}<0
\end{aligned}
$$

These equalities proves that: $x_{G}^{1}<x_{B}^{1}, x_{G}^{2}>x_{B}^{2}$ and $P_{G}>P_{B}$.

We also prove very easily that:

$$
\begin{aligned}
\frac{\partial E[P]}{\partial V[y]} & =\frac{1-\pi_{B B}}{2-\pi_{G G}-\pi_{B B}} \frac{\partial P_{G}}{\partial V[y]}+\frac{1-\pi_{G G}}{2-\pi_{G G}-\pi_{B B}} \frac{\partial P_{B}}{\partial V[y]}=0 \\
& =\frac{\partial E\left[x^{1}\right]}{\partial V[y]}=\frac{\partial E\left[x^{2}\right]}{\partial V[y]}
\end{aligned}
$$


Finally, we prove the following result regarding the impact of the variance:

$$
\begin{aligned}
\frac{\partial V[P]}{\partial V[y]} & =\frac{\left(1-\pi_{B B}\right)\left(1-\pi_{G G}\right)}{\left(2-\pi_{G G}-\pi_{B B}\right)^{2}} 2\left(y_{G}-y_{B}\right)\left(\frac{\partial P_{G}}{\partial V[y]}-\frac{\partial P_{B}}{\partial V[y]}\right) \frac{P_{G}-P_{B}}{y_{G}-y_{B}} \\
& \approx \frac{\left(\pi_{G G}+\pi_{B B}-1\right) \beta \widehat{M}}{1-\left(\pi_{G G}+\pi_{B B}-1\right) \beta \widehat{M}} \frac{\left(1-\pi_{B B}\right)\left(1-\pi_{G G}\right)}{2-\pi_{G G}-\pi_{B B}}\left(\frac{1}{1-\pi_{B B}}+\frac{1}{1-\pi_{G G}}\right)\left(\frac{\partial P_{G}}{\partial y_{G}}-\frac{\partial P_{G}}{\partial y_{B}}+\frac{\partial P_{B}}{\partial y_{B}}-\frac{\partial P_{B}}{\partial y_{G}}\right) \\
& =2\left(\frac{\left(\pi_{G G}+\pi_{B B}-1\right) \beta \widehat{M}}{1-\left(\pi_{G G}+\pi_{B B}-1\right) \beta \widehat{M}}\right)^{2}
\end{aligned}
$$

And analogously $(i=1,2)$ :

$$
(P+y)^{2} \frac{\partial V\left[x^{i}\right]}{\partial V[y]}=\left(\frac{\kappa}{\eta^{i} \beta \widehat{M}}\right)^{2} \frac{\partial V[P]}{\partial V[y]}=2\left(\frac{1}{\eta^{i}} \frac{\left(\pi_{G G}+\pi_{B B}-1\right) \kappa}{1-\left(\pi_{G G}+\pi_{B B}-1\right) \beta \widehat{M}}\right)^{2}
$$

This concludes the proof of Proposition 2

\section{Proof of proposition 3}

We study the evolution of prices and quantities around the certain equilibrium $\alpha^{1}=\alpha^{2}=\alpha$, where the asset prices are $P_{G}$ and $P_{B}$ and the agents' asset holdings are denoted respectively $x_{G}$ and $x_{B}$. This equilibrium variables are defined as $(k=B, G)$ :

$$
P_{k}=\beta \sum_{j=B, G} \pi_{k, j}\left(1+\alpha\left(u^{\prime}\left(\delta+\left(P_{j}+y_{j}\right) \frac{V}{\eta^{1}+\eta^{2}}\right)-1\right)\right)\left(P_{j}+y_{j}\right)
$$

and: $\left(\eta^{1}+\eta^{2}\right) x_{k}=V$

\section{C.1 Quantities}

We derive the equation 30 linking $x_{k}^{1}$ and $x_{k}^{2}$ relative to $\alpha^{l}(l=1,2)$ in the vicinity of the symmetric equilibrium:

$$
\begin{aligned}
& \sum_{j=B, G} \pi_{k, j}\left(1+\alpha\left(u^{\prime}\left(\delta+\left(P_{j}+y_{j}\right) \frac{V}{\eta^{1}+\eta^{2}}\right)-1\right)\right) \frac{\partial P_{j}}{\partial \alpha^{l}}+1_{l=1} \pi_{k, j}\left(u^{\prime}\left(\delta+\left(P_{j}+y_{j}\right) \frac{V}{\eta^{1}+\eta^{2}}\right)-1\right)\left(P_{j}+y_{j}\right) \\
& +\alpha \sum_{j=B, G} \pi_{k, j} u^{\prime \prime}\left(\delta+\left(P_{j}+y_{j}\right) \frac{V}{\eta^{1}+\eta^{2}}\right)\left(P_{j}+y_{j}\right)\left(\frac{V}{\eta^{1}+\eta^{2}} \frac{\partial P_{j}}{\partial \alpha^{l}}+\left(P_{j}+y_{j}\right) \frac{\partial x_{k}^{1}}{\partial \alpha^{l}}\right) \\
= & \sum_{j=B, G} \pi_{k, j}\left(1+\alpha\left(u^{\prime}\left(\delta+\left(P_{j}+y_{j}\right) \frac{V}{\eta^{1}+\eta^{2}}\right)-1\right)\right) \frac{\partial P_{j}}{\partial \alpha^{l}}+1_{l=2} \pi_{k, j}\left(u^{\prime}\left(\delta+\left(P_{j}+y_{j}\right) \frac{V}{\eta^{1}+\eta^{2}}\right)-1\right)\left(P_{j}+y_{j}\right) \\
& +\alpha \sum_{j=B, G} \pi_{k, j} u^{\prime \prime}\left(\delta+\left(P_{j}+y_{j}\right) \frac{V}{\eta^{1}+\eta^{2}}\right)\left(P_{j}+y_{j}\right)\left(\frac{V}{\eta^{1}+\eta^{2}} \frac{\partial P_{j}}{\partial \alpha^{l}}+\left(P_{j}+y_{j}\right) \frac{\partial x_{k}^{2}}{\partial \alpha^{l}}\right)
\end{aligned}
$$

or after simplification:

$$
\begin{aligned}
& \alpha \sum_{j=B, G} \pi_{k, j} u^{\prime \prime}\left(\delta+\left(P_{j}+y_{j}\right) \frac{V}{\eta^{1}+\eta^{2}}\right)\left(P_{j}+y_{j}\right)^{2}\left(\frac{\partial x_{k}^{1}}{\partial \alpha^{l}}-\frac{\partial x_{k}^{2}}{\partial \alpha^{l}}\right) \\
= & \left(1_{l=2}-1_{l=1}\right) \sum_{j=B, G} \pi_{k, j}\left(u^{\prime}\left(\delta+\left(P_{j}+y_{j}\right) \frac{V}{\eta^{1}+\eta^{2}}\right)-1\right)\left(P_{j}+y_{j}\right)
\end{aligned}
$$


Using the market clearing condition, we deduce:

$$
\eta^{1} \frac{\partial x_{k}^{1}}{\partial \alpha^{l}}=-\eta^{2} \frac{\partial x_{k}^{2}}{\partial \alpha^{l}}=\left(1_{l=2}-1_{l=1}\right) \frac{\eta^{1} \eta^{2}}{\eta^{1}+\eta^{2}} \frac{\sum_{j=B, G} \pi_{k, j}\left(u^{\prime}\left(\delta+\left(P_{j}+y_{j}\right) \frac{V}{\eta^{1}+\eta^{2}}\right)-1\right)\left(P_{j}+y_{j}\right)}{\alpha \sum_{j=B, G} \pi_{k, j} u^{\prime \prime}\left(\delta+\left(P_{j}+y_{j}\right) \frac{V}{\eta^{1}+\eta^{2}}\right)\left(P_{j}+y_{j}\right)^{2}}
$$

We deduce that $\frac{\partial x_{k}^{1}}{\partial \alpha^{l}}>0\left(u^{\prime \prime}<0\right)$ for $k=B, G$. Moreover, we have $\frac{\partial x_{B}^{1}}{\partial \alpha^{l}}>\frac{\partial x_{G}^{1}}{\partial \alpha^{l}}$ iif:

$$
\begin{array}{r}
-\frac{\sum_{j=B, G} \pi_{B, j}\left(u^{\prime}\left(\delta+\left(P_{j}+y_{j}\right) \frac{V}{\eta^{1}+\eta^{2}}\right)-1\right)\left(P_{j}+y_{j}\right)}{\sum_{j=B, G} \pi_{B, j} u^{\prime \prime}\left(\delta+\left(P_{j}+y_{j}\right) \frac{V}{\eta^{1}+\eta^{2}}\right)\left(P_{j}+y_{j}\right)^{2}}>-\frac{\sum_{j=B, G} \pi_{G, j}\left(u^{\prime}\left(\delta+\left(P_{j}+y_{j}\right) \frac{V}{\eta^{1}+\eta^{2}}\right)-1\right)\left(P_{j}+y_{j}\right)}{\sum_{j=B, G} \pi_{G, j} u^{\prime \prime}\left(\delta+\left(P_{j}+y_{j}\right) \frac{V}{\eta^{1}+\eta^{2}}\right)\left(P_{j}+y_{j}\right)^{2}} \\
-\left(\sum_{j=B, G} \pi_{G, j} u^{\prime \prime}\left(\delta+\left(P_{j}+y_{j}\right) \frac{V}{\eta^{1}+\eta^{2}}\right)\left(P_{j}+y_{j}\right)^{2}\right)\left(\sum_{j=B, G} \pi_{B, j}\left(u^{\prime}\left(\delta+\left(P_{j}+y_{j}\right) \frac{V}{\eta^{1}+\eta^{2}}\right)-1\right)\left(P_{j}+y_{j}\right)\right)> \\
-\left(\sum_{j=B, G} \pi_{G, j}\left(u^{\prime}\left(\delta+\left(P_{j}+y_{j}\right) \frac{V}{\eta^{1}+\eta^{2}}\right)-1\right)\left(P_{j}+y_{j}\right)\right)\left(\sum_{j=B, G} \pi_{B, j} u^{\prime \prime}\left(\delta+\left(P_{j}+y_{j}\right) \frac{V}{\eta^{1}+\eta^{2}}\right)\left(P_{j}+y_{j}\right)^{2}\right) \\
\left(\left(1-\pi_{B B}\right)\left(1-\pi_{G G}\right)-\pi_{G G} \pi_{B B}\right) u^{\prime \prime}\left(\delta+\left(P_{G}+y_{G}\right) \frac{V}{\eta^{1}+\eta^{2}}\right)\left(P_{G}+y_{G}\right)^{2}\left(u^{\prime}\left(\delta+\left(P_{B}+y_{B}\right) \frac{V}{\eta^{1}+\eta^{2}}\right)-1\right)\left(P_{B}+y_{B}\right)> \\
\left(\left(1-\pi_{B B}\right)\left(1-\pi_{G G}\right)-\pi_{G G} \pi_{B B}\right) u^{\prime \prime}\left(\delta+\left(P_{B}+y_{B}\right) \frac{V}{\eta^{1}+\eta^{2}}\right)\left(P_{B}+y_{B}\right)^{2}\left(u^{\prime}\left(\delta+\left(P_{G}+y_{G}\right) \frac{V}{\eta^{1}+\eta^{2}}\right)-1\right)\left(P_{G}+y_{G}\right) \\
\left.-\frac{u^{\prime \prime}\left(\delta+\left(P_{G}+y_{G}\right) \frac{V}{\eta^{1}+\eta^{2}}\right)}{u^{\prime}\left(\delta+\left(P_{G}+y_{G}\right) \frac{V}{\eta^{1}+\eta^{2}}\right)-1}\left(P_{G}+y_{G}\right)>-\frac{V}{u^{\prime}\left(\delta+\left(P_{B}+y_{B}\right) \frac{V}{\eta^{1}+\eta^{2}}\right)}\left(P_{B}\right) \frac{V}{\eta^{1}+\eta^{2}}\right)-1
\end{array}
$$

A sufficient condition for $\frac{\partial x_{B}^{1}}{\partial \alpha^{l}}>\frac{\partial x_{G}^{1}}{\partial \alpha^{l}}$ is $x \mapsto-x \frac{u^{\prime \prime}(\delta+x)}{u^{\prime}(\delta+x)-1}$.

\section{C.2 Prices}

$$
\begin{aligned}
\frac{\partial P_{k}}{\partial \alpha^{l}} & =1_{l=i} \beta \sum_{j=B, G} \pi_{k, j}\left(u^{\prime}\left(\delta+\left(P_{j}+y_{j}\right) \frac{V}{\eta^{1}+\eta^{2}}\right)-1\right)\left(P_{j}+y_{j}\right) \\
& +\beta \sum_{j=B, G} \pi_{k, j}\left(1+\alpha\left(u^{\prime}\left(\delta+\left(P_{j}+y_{j}\right) \frac{V}{\eta^{1}+\eta^{2}}\right)-1\right)\right) \frac{\partial P_{j}}{\partial \alpha^{l}} \\
& +\beta \alpha \sum_{j=B, G} \pi_{k, j} u^{\prime \prime}\left(\delta+\left(P_{j}+y_{j}\right) \frac{V}{\eta^{1}+\eta^{2}}\right)\left(P_{j}+y_{j}\right)\left(\left(P_{j}+y_{j}\right) \frac{\partial x_{k}^{i}}{\partial \alpha^{l}}+\frac{V}{\eta^{1}+\eta^{2}} \frac{\partial P_{j}}{\partial \alpha^{l}}\right) \\
& =1_{l=i} \beta \sum_{j=B, G} \pi_{k, j}\left(u^{\prime}\left(\delta+\left(P_{j}+y_{j}\right) \frac{V}{\eta^{1}+\eta^{2}}\right)-1\right)\left(P_{j}+y_{j}\right) \\
& +\beta \sum_{j=B, G} \pi_{k, j}\left(1+\alpha\left(u^{\prime}\left(\delta+\left(P_{j}+y_{j}\right) \frac{V}{\eta^{1}+\eta^{2}}\right)-1\right)\left(1+\frac{\left(P_{j}+y_{j}\right) V}{\eta^{1}+\eta^{2}} \frac{u^{\prime \prime}\left(\delta+\left(P_{j}+y_{j}\right) \frac{V}{\eta^{1}+\eta^{2}}\right)}{u^{\prime}\left(\delta+\frac{\left(P_{j}+y_{j}\right) V}{\eta^{1}+\eta^{2}}\right)-1}\right)\right) \frac{\partial P_{j}}{\partial \alpha^{l}} \\
& +\beta \alpha \sum_{j=B, G} \pi_{k, j} u^{\prime \prime}\left(\delta+\left(P_{j}+y_{j}\right) \frac{V}{\eta^{1}+\eta^{2}}\right)\left(P_{j}+y_{j}\right)^{2} \frac{\partial x_{k}^{i}}{\partial \alpha^{l}}
\end{aligned}
$$


Using the expression of $\frac{\partial x_{k}^{i}}{\partial \alpha^{l}}$, we obtain:

$$
\begin{aligned}
\frac{\partial P_{k}}{\partial \alpha^{l}} & =\left(1_{l=i}+\frac{(-1)^{i}}{\eta^{i}}\left(1_{l=1}-1_{l=2}\right) \frac{\eta^{1} \eta^{2}}{\eta^{1}+\eta^{2}}\right) \beta \sum_{j=B, G} \pi_{k, j}\left(u^{\prime}\left(\delta+\left(P_{j}+y_{j}\right) \frac{V}{\eta^{1}+\eta^{2}}\right)-1\right)\left(P_{j}+y_{j}\right) \\
& +\beta \sum_{j=B, G} \pi_{k, j}\left(1+\alpha\left(u^{\prime}\left(\delta+\left(P_{j}+y_{j}\right) \frac{V}{\eta^{1}+\eta^{2}}\right)-1\right)\left(1+\frac{\left(P_{j}+y_{j}\right) V}{\eta^{1}+\eta^{2}} \frac{u^{\prime \prime}\left(\delta+\left(P_{j}+y_{j}\right) \frac{V}{\eta^{1}+\eta^{2}}\right)}{u^{\prime}\left(\delta+\frac{\left(P_{j}+y_{j}\right) V}{\eta^{1}+\eta^{2}}\right)-1}\right)\right) \frac{\partial P_{j}}{\partial \alpha^{l}} \\
& =\frac{\eta^{1} 1_{l=1}+\eta^{2} 1_{l=2}}{\eta^{1}+\eta^{2}} \beta \sum_{j=B, G} \pi_{k, j}\left(u^{\prime}\left(\delta+\left(P_{j}+y_{j}\right) \frac{V}{\eta^{1}+\eta^{2}}\right)-1\right)\left(P_{j}+y_{j}\right)+\beta \sum_{j=B, G} \pi_{k, j} M_{j} \frac{\partial P_{j}}{\partial \alpha^{l}} \\
& =\beta \frac{\eta^{1} 1_{l=1}+\eta^{2} 1_{l=2}}{\eta^{1}+\eta^{2}} \sum_{j=B, G} \pi_{k, j} \Delta_{j}+\beta \sum_{j=B, G} \pi_{k, j} M_{j} \frac{\partial P_{j}}{\partial \alpha^{l}}
\end{aligned}
$$

with: $M_{j}=1+\alpha\left(u^{\prime}\left(\delta+\left(P_{j}+y_{j}\right) \frac{V}{\eta^{1}+\eta^{2}}\right)-1\right)\left(1+\frac{\left(P_{j}+y_{j}\right) V}{\eta^{1}+\eta^{2}} \frac{u^{\prime \prime}\left(\delta+\left(P_{j}+y_{j}\right) \frac{V}{\eta^{1}+\eta^{2}}\right)}{u^{\prime}\left(\delta+\frac{\left(P_{j}+y_{j}\right) V}{\eta^{1}+\eta^{2}}\right)-1}\right) \quad j=B, G$

$$
\Delta_{j}=\left(u^{\prime}\left(\delta+\left(P_{j}+y_{j}\right) \frac{V}{\eta^{1}+\eta^{2}}\right)-1\right)\left(P_{j}+y_{j}\right)
$$

Using matrix notations, we have:

$$
\begin{gathered}
{\left[\begin{array}{cc}
1-\beta M_{G} \pi_{G G} & -\beta M_{B}\left(1-\pi_{G G}\right) \\
-\beta M_{G}\left(1-\pi_{B B}\right) & 1-\beta M_{B} \pi_{B B}
\end{array}\right]\left[\begin{array}{c}
\frac{\partial P_{G}}{\partial \alpha^{l}} \\
\frac{\partial P_{B}}{\partial \alpha^{l}}
\end{array}\right]=\beta \frac{\eta^{1} 1_{l=1}+\eta^{2} 1_{l=2}}{\eta^{1}+\eta^{2}}\left[\begin{array}{c}
\pi_{G G} \Delta_{G}+\left(1-\pi_{G G}\right) \Delta_{B} \\
\left(1-\pi_{B B}\right) \Delta_{G}+\pi_{B B} \Delta_{B}
\end{array}\right]} \\
\left(1-\beta \pi_{G G} M_{G}-\beta \pi_{B B} M_{B}+\beta^{2}\left(\pi_{G G}+\pi_{B B}-1\right) M_{G} M_{B}\right)\left[\begin{array}{c}
\frac{\partial P_{G}}{\partial \alpha^{l}} \\
\frac{\partial P_{B}}{\partial \alpha^{l}}
\end{array}\right]= \\
\beta \frac{\eta^{1} 1_{l=1}+\eta^{2} 1_{l=2}}{\eta^{1}+\eta^{2}}\left[\begin{array}{cc}
1-\beta M_{B} \pi_{B B} & \beta M_{B}\left(1-\pi_{G G}\right) \\
\beta M_{G}\left(1-\pi_{B B}\right) & 1-\beta M_{G} \pi_{G G}
\end{array}\right]\left[\begin{array}{c}
\pi_{G G} \Delta_{G}+\left(1-\pi_{G G}\right) \Delta_{B} \\
\left(1-\pi_{B B}\right) \Delta_{G}+\pi_{B B} \Delta_{B}
\end{array}\right] \\
\beta \frac{\eta^{1} 1_{l=1}+\eta^{2} 1_{l=2}}{\eta^{1}+\eta^{2}}\left[\begin{array}{l}
\left(\pi_{G G}-\beta M_{B}\left(\pi_{G G}+\pi_{B B}-1\right)\right) \Delta_{G}+\left(1-\pi_{G G}\right) \Delta_{B} \\
\left(\pi_{B B}-\beta M_{G}\left(\pi_{G G}+\pi_{B B}-1\right)\right) \Delta_{B}+\left(1-\pi_{B B}\right) \Delta_{G}
\end{array}\right]>0
\end{gathered}
$$

To prove the last result, notice that the sign of $\frac{\partial P_{G}}{\partial \alpha^{l}}-\frac{\partial P_{B}}{\partial \alpha^{l}}$ is the sign of $\left(1-\beta M_{B}\right) \Delta_{G}-\left(1-\beta M_{G}\right) \Delta_{B}$, which is always positive. Indeed for small volumes $V \approx 0$, this expression collapses to $\left(1-\beta\left(1+\alpha\left(u^{\prime}(\delta)-1\right)\right)\right)\left(u^{\prime}(\delta)-\right.$ 1) $\left(P_{G}+y_{G}-P_{B}-y_{B}\right)>0$.

\section{Proof of Proposition 4}

We introduce an option in the economy with a single asset defined by $\left(X^{*}, Y^{*}\right)$. We suppose that $P>K$ : the option has a strictly positive price, but is not traded.

As in the no option economy, we define $X=\left(y_{B}, y_{G}, V, \alpha^{1}, \alpha^{2}\right) \in\left(\mathbb{R}^{+}\right)^{5}$ the vector of parameters and $Z Z=$ $\left(P_{B}, P_{G}, Q_{B}, Q_{G}, x_{B}^{1}, x_{G}^{1}, x_{B}^{2}, x_{G}^{2}, s_{B}^{1}, s_{G}^{1}, s_{B}^{2}, s_{G}^{2}\right) \in\left(\mathbb{R}^{+}\right)^{12}$ the vector of endogenous variables.

We define the vector $G$ of length 12 of equations as $(i=1,2$ and $k=1,2-k=B, G$ refers to the aggregate 
state and $i$ to the type):

$$
\begin{aligned}
G_{2(i-1)+k}(Z, X) & =P_{k}-\beta \sum_{j=B, G} \pi_{k, j}\left(1+\alpha^{i}\left(u^{\prime}\left(\delta+\left(P_{j}+y_{j}\right) x_{k}^{i}+\left(P_{j}-K\right)^{+} s_{k}^{i}\right)-1\right)\right)\left(P_{j}+y_{j}\right) \\
G_{4+k}(Z, X) & =\eta^{1} x_{k}^{1}+\eta^{2} x_{k}^{2}-V \\
G_{6+2(i-1)+k}(Z, X) & =Q_{k}-\beta \sum_{j=B, G} \pi_{k, j}\left(1+\alpha^{i}\left(u^{\prime}\left(\delta+\left(P_{j}+y_{j}\right) x_{k}^{i}+\left(P_{j}-K\right)^{+} s_{k}^{i}\right)-1\right)\right)\left(P_{j}-K\right)^{+} \\
G_{10+k}(Z, X) & =\eta^{1} s_{k}^{1}+\eta^{2} s_{k}^{2}
\end{aligned}
$$

$G$ stacks pricing functions for both agents' types and the market equilibrium. The first 4 equations are the asset pricing equations, the next 2 ones the asset market equilibrium, the next 4 the option pricing, and the last 2 the option market clearing.

For a given set of parameters $X$, the equilibrium is defined as:

$$
G(Z Z, X)=0
$$

We already know that an equilibrium exists for $X^{*}=\left(y_{B}, y_{G}, V^{*}, \alpha, \alpha\right)$, which is $Z Z^{*}=\left(P_{B}, P_{G}, Q_{B}, Q_{G}, x_{B}, x_{G}, x_{B}, x_{G}, 0_{4}\right)$. We use again the implicit function theorem to prove the equilibrium existence in the vicinity of $\left(X^{*}, Z Z^{*}\right)$. We need therefore to compute derivatives in $\left(X^{*}, Z Z^{*}\right)$. We compute now the Jacobian.

- The first four equations:

$$
\begin{aligned}
\frac{\partial G_{2(i-1)+k}}{\partial P_{l}}\left(Z^{*}, X^{*}\right) & =1_{k=l}-\beta \sum_{j=B, G} \pi_{k, j}\left(1+\alpha\left(u^{\prime}\left(\delta+\left(P_{j}+y_{j}\right) x_{k}\right)-1\right)\right) 1_{j=l} \\
& -\beta \alpha \sum_{j=B, G} \pi_{k, j} u^{\prime \prime}\left(\delta+\left(P_{j}+y_{j}\right) x_{k}\right)\left(P_{j}+y_{j}\right) x_{k} 1_{j=l} \\
\frac{\partial G_{2(i-1)+k}}{\partial x_{l}^{i^{\prime}}}\left(Z^{*}, X^{*}\right) & =-1_{i=i^{\prime}} 1_{l=k} \beta \alpha \sum_{j=B, G} \pi_{k, j} u^{\prime \prime}\left(\delta+\left(P_{j}+y_{j}\right) x_{k}\right)\left(P_{j}+y_{j}\right)^{2} \\
\frac{\partial G_{2(i-1)+k}}{\partial Q_{l}}\left(Z^{*}, X^{*}\right) & =0 \\
\frac{\partial G_{2(i-1)+k}}{\partial s_{l}^{i^{\prime}}}\left(Z^{*}, X^{*}\right) & =-1_{i=i^{\prime}} 1_{l=k} \beta \alpha \pi_{k, G} u^{\prime \prime}\left(\delta+\left(P_{G}+y_{G}\right) x_{k}\right)\left(P_{G}+y_{G}\right)\left(P_{G}-K\right)
\end{aligned}
$$

- The next two equations:

$$
\begin{aligned}
& \frac{\partial G_{4+k}}{\partial P_{l}}\left(Z^{*}, X^{*}\right)=0 \\
& \frac{\partial G_{4+k}}{\partial x_{l}^{i^{\prime}}}\left(Z^{*}, X^{*}\right)=\eta^{i^{\prime}} 1_{k=l} \\
& \frac{\partial G_{4+k}}{\partial Q_{l}}\left(Z^{*}, X^{*}\right)=0 \\
& \frac{\partial G_{4+k}}{\partial s_{l}^{i^{\prime}}}\left(Z^{*}, X^{*}\right)=0
\end{aligned}
$$


- The next four equations:

$$
\begin{aligned}
& \frac{\partial G_{6+2(i-1)+k}}{\partial P_{l}}\left(Z^{*}, X^{*}\right)=-1_{l=2} \beta \pi_{k, G}\left(1+\alpha\left(u^{\prime}\left(\delta+\left(P_{G}+y_{G}\right) x_{k}\right)-1\right)\right) 1_{l=2} \\
& -\beta \alpha \pi_{k, G} u^{\prime \prime}\left(\delta+\left(P_{G}+y_{G}\right) x_{k}\right)\left(P_{G}-K\right) x_{k} \\
& \frac{\partial G_{6+2(i-1)+k}}{\partial x_{l}^{i^{\prime}}}\left(Z^{*}, X^{*}\right)=-1_{i=i^{\prime}} 1_{k=l} \beta \alpha \pi_{k, G} u^{\prime \prime}\left(\delta+\left(P_{G}+y_{G}\right) x_{k}\right)\left(P_{G}+y_{G}\right)\left(P_{G}-K\right) \\
& \frac{\partial G_{6+2(i-1)+k}}{\partial Q_{l}}\left(Z^{*}, X^{*}\right)=1_{l=k} \\
& \frac{\partial G_{6+2(i-1)+k}}{\partial s_{l}^{i^{\prime}}}\left(Z^{*}, X^{*}\right)=-1_{i=i^{\prime}} 1_{k=l} \beta \alpha \pi_{k, G} u^{\prime \prime}\left(\delta+\left(P_{G}+y_{G}\right) x_{k}\right)\left(P_{G}-K\right)^{2}
\end{aligned}
$$

- The last two equations:

$$
\begin{aligned}
& \frac{\partial G_{10+k}}{\partial P_{l}}\left(Z^{*}, X^{*}\right)=0 \\
& \frac{\partial G_{10+k}}{\partial x_{l}^{i^{\prime}}}\left(Z^{*}, X^{*}\right)=0 \\
& \frac{\partial G_{10+k}}{\partial Q_{l}}\left(Z^{*}, X^{*}\right)=0 \\
& \frac{\partial G_{10+k}}{\partial s_{l}^{i^{\prime}}}\left(Z^{*}, X^{*}\right)=\eta^{i^{\prime}} 1_{k=l}
\end{aligned}
$$

- The Jacobian has the following expression:

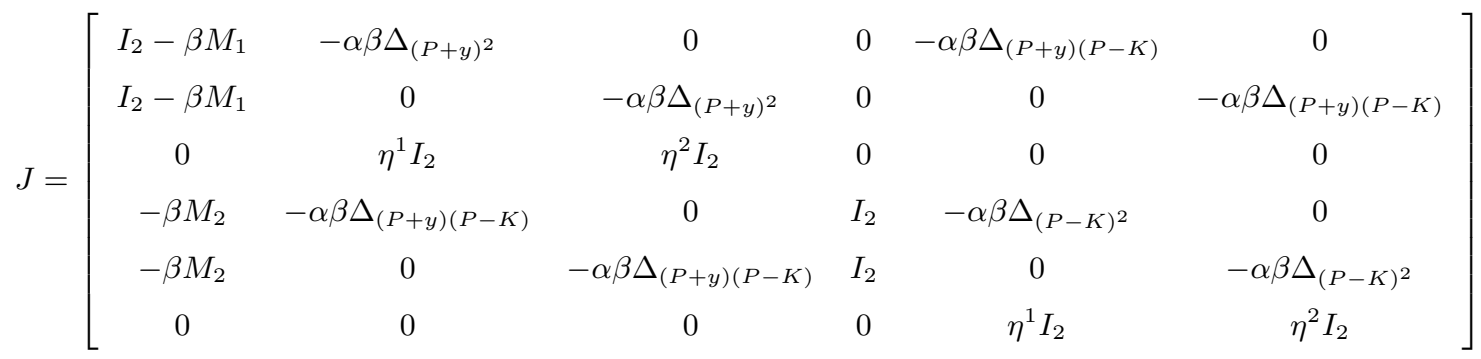

with $T=\left(\pi_{k, l}\right), \Delta_{M, i}=\operatorname{diag}\left(0_{i-1},\left(1+\alpha\left(u^{\prime}\left(\delta+\left(P_{j}+y_{j}\right) x_{j}\right)-1\right)-\alpha u^{\prime \prime}\left(\delta+\left(P_{j}+y_{j}\right) x_{j}\right)\left(P_{j}-K\right) x_{j}\right){ }_{j=i, \ldots, 2}\right)$, and:

$$
\begin{aligned}
m_{k, j} & =1+\alpha\left(u^{\prime}\left(\delta+\left(P_{j}+y_{j}\right) x_{k}\right)-1\right)-\alpha u^{\prime \prime}\left(\delta+\left(P_{j}+y_{j}\right) x_{k}\right)\left(P_{j}-K\right) x_{k} \\
M_{i} & =\left(\pi_{k, j} m_{k, j}\right) \operatorname{diag}\left(0_{i-1},(1)_{j=i, 2}\right) \\
\Delta_{S T} & =\operatorname{diag}\left(\left(\sum_{j=B, G} \pi_{k, j} u^{\prime \prime}\left(\delta+\left(P_{j}+y_{j}\right) x_{k}\right) S_{j} T_{j}\right)_{k=1,2}\right)
\end{aligned}
$$

with: $\quad S_{j}$ and $T_{j}$ payoffs in state $j$, e.g. $\left(P_{B}-K\right)^{+}=0$ 
- Let $X=\left(X_{1}, \ldots, X_{6}\right) \in \operatorname{ker}(J)$. We deduce the following equations:

$$
\begin{aligned}
\left(I_{2}-\beta M_{1}\right) X_{1}-\alpha \beta T \Delta_{(P+y)^{2}} X_{2}-\alpha \beta T \Delta_{(P+y)(P-K)} X_{5} & =0 \\
\left(I_{2}-\beta T M_{1}\right) X_{1}-\alpha \beta T \Delta_{(P+y)^{2}} X_{3}-\alpha \beta T \Delta_{(P+y)(P-K)} X_{6} & =0 \\
-\beta M_{2} X_{1}-\alpha \beta T \Delta_{(P+y)(P-K)} X_{2}+X_{4}-\alpha \beta T \Delta_{(P-K)^{2}} X_{5} & =0 \\
-\beta M_{2} X_{1}-\alpha \beta T \Delta_{(P+y)(P-K)} X_{3}+X_{4}-\alpha \beta T \Delta_{(P-K)^{2}} X_{6} & =0 \\
\eta^{1} X_{2}+\eta^{2} X_{3} & =0 \\
\eta^{1} X_{5}+\eta^{2} X_{6} & =0
\end{aligned}
$$

A linear combination of the first two lines gives $\left(I_{2}-\beta T \Delta_{M, 1}\right) X_{1}=0$, or $X_{1}=0$ since $I_{2}-\beta T \Delta_{M, 1}$ is invertible. Analogously we deduce $X_{4}=0$. The previous system simplifies into $\left(X_{3}\right.$ is collinear to $X_{2}$ and $X_{6}$ to $X_{5}$ and $\left.\alpha \beta>0\right)$ :

$$
\begin{gathered}
\Delta_{(P+y)^{2}} X_{2}+\Delta_{(P+y)(P-K)} X_{5}=0 \\
\Delta_{(P+y)(P-K)} X_{2}+\Delta_{(P-K)^{2}} X_{5}=0
\end{gathered}
$$

We obtain finally $(i=1,2)$ :

$$
\begin{gathered}
\sum_{j=B, G} \pi_{k, j} u^{\prime \prime}\left(\delta+\left(P_{j}+y_{j}\right) x_{i}\right)\left(P_{j}+y_{j}\right)^{2} X_{2, i}+\pi_{k, G} u^{\prime \prime}\left(\delta+\left(P_{G}+y_{G}\right) x_{i}\right)\left(P_{G}+y_{G}\right)\left(P_{G}-K\right) X_{5, i}=0 \\
\pi_{k, G} u^{\prime \prime}\left(\delta+\left(P_{G}+y_{G}\right) x_{i}\right)\left(P_{G}+y_{G}\right)\left(P_{G}-K\right) X_{2, i}+\pi_{k, G} u^{\prime \prime}\left(\delta+\left(P_{G}+y_{G}\right) x_{i}\right)\left(P_{G}-K\right)^{2} X_{5, i}=0
\end{gathered}
$$

By difference (after multiplication of the last line by $\frac{P_{G}+y_{G}}{P_{G}-K}>0$ ), we obtain easily:

$$
\begin{array}{r}
\pi_{k, B} u^{\prime \prime}\left(\delta+\left(P_{B}+y_{B}\right) x_{i}\right)\left(P_{j}+y_{B}\right)^{2} X_{2, i}=0 \\
\pi_{k, G} u^{\prime \prime}\left(\delta+\left(P_{G}+y_{G}\right) x_{i}\right)\left(P_{G}+y_{G}\right)\left(P_{G}-K\right) X_{2, i}+\pi_{k, G} u^{\prime \prime}\left(\delta+\left(P_{G}+y_{G}\right) x_{i}\right)\left(P_{G}-K\right)^{2} X_{5, i}=0
\end{array}
$$

We deduce that $X_{2}=X_{5}=X_{3}=X_{6}=0=X_{1}=X_{4}$. In consequence, ker $J=0_{6}$ and the function $G$ is invertible in the vicinity of $\left(Z Z^{*}, Y^{*}\right)$, and our equilibrium exists. 


\section{E Proof of Proposition 5}

Prices for the asset and the option express as $(k=1,2$ and $i=1,2)$ :

$$
\begin{aligned}
P_{k} & =\beta \pi_{k, G}\left(1+\alpha^{i}\left(u^{\prime}\left(\delta+\left(P_{G}+y_{G}\right) x_{k}^{i}+\left(P_{G}-K\right) s_{k}^{i}\right)-1\right)\right)\left(P_{G}+y_{G}\right) \\
& +\beta \pi_{k, B}\left(1+\alpha^{i}\left(u^{\prime}\left(\delta+\left(P_{B}+y_{B}\right) x_{k}^{i}\right)-1\right)\right)\left(P_{B}+y_{B}\right) \\
Q_{k} & =\beta \pi_{k, G}\left(1+\alpha^{i}\left(u^{\prime}\left(\delta+\left(P_{G}+y_{G}\right) x_{k}^{i}+\left(P_{G}-K\right) s_{k}^{i}\right)-1\right)\right)\left(P_{G}-K\right)
\end{aligned}
$$

with:

$$
\begin{aligned}
& \alpha^{1}\left(u^{\prime}\left(\delta+\left(P_{G}+y_{G}\right) x_{k}^{1}+\left(P_{G}-K\right) s_{k}^{1}\right)-1\right)=\alpha^{2}\left(u^{\prime}\left(\delta+\left(P_{G}+y_{G}\right) x_{k}^{2}+\left(P_{G}-K\right) s_{k}^{2}\right)-1\right) \\
& \alpha^{1}\left(u^{\prime}\left(\delta+\left(P_{B}+y_{B}\right) x_{k}^{1}\right)-1\right)=\alpha^{2}\left(u^{\prime}\left(\delta+\left(P_{B}+y_{B}\right) x_{k}^{2}\right)-1\right)
\end{aligned}
$$

and:

$$
\begin{aligned}
& \eta^{1} x_{k}^{1}+\eta^{1} x_{k}^{2}=V \\
& \eta^{1} s_{k}^{1}+\eta^{1} s_{k}^{2}=0
\end{aligned}
$$

It is straightforward that asset and options quantities $x_{k}^{i}$ and $s_{k}^{i}$ do not depend on the state of the world $k$. We note them respectively $x^{i}$ and $s^{i}$ for $i=1,2$.

We study the evolution of endogenous variables in the vicinity of the certain equilibrium, where $y_{G}=y_{B}=y$, $P_{k}=P, Q_{k}=Q, s^{1}=0$ (the option is priced but not traded). This is simply the certain economy of Section B. where a non-traded option is introduced.

Results of Proposition 5 are straightforward in the vicinity of $y_{G}=y_{B}=y$ :

$$
\begin{aligned}
& \alpha^{1}\left(u^{\prime}\left(\delta+\left(P_{G}+y_{G}\right) x^{1}+\left(P_{G}-K\right) s^{1}\right)-1\right)=\alpha^{2}\left(u^{\prime}\left(\delta+\left(P_{G}+y_{G}\right) x^{2}+\left(P_{G}-K\right) s^{2}\right)-1\right) \\
& \alpha^{1}\left(u^{\prime}\left(\delta+\left(P_{B}+y_{B}\right) x^{1}\right)-1\right)=\alpha^{2}\left(u^{\prime}\left(\delta+\left(P_{B}+y_{B}\right) x^{2}\right)-1\right)
\end{aligned}
$$

Since $\alpha^{1}>\alpha^{2}$, the second equality implies $u^{\prime}\left(\delta+\left(P_{B}+y_{B}\right) x^{1}\right)<u^{\prime}\left(\delta+\left(P_{B}+y_{B}\right) x^{2}\right)$ and therefore $x^{1}>x^{2}$ since $u^{\prime}$ is decreasing.

We additionally have $s^{1}<0$ since $\alpha^{1}\left(u^{\prime}\left(\delta+\left(P_{G}+y_{G}\right) x^{1}\right)-1\right)<\alpha^{2}\left(u^{\prime}\left(\delta+\left(P_{G}+y_{G}\right) x^{2}\right)-1\right)$. Indeed, it is equivalent to:

$$
\frac{u^{\prime}\left(\delta+\left(P_{G}+y_{G}\right) x^{1}\right)-1}{u^{\prime}\left(\delta+\left(P_{G}+y_{G}\right) x^{2}\right)-1}<\frac{u^{\prime}\left(\delta+\left(P_{B}+y_{B}\right) x^{1}\right)-1}{u^{\prime}\left(\delta+\left(P_{B}+y_{B}\right) x^{2}\right)-1}
$$

It is sufficient that $\pi \mapsto \frac{u^{\prime}\left(\delta+\pi x^{1}\right)-1}{u^{\prime}\left(\delta+\pi x^{2}\right)-1}$ is decreasing, or after derivation that:

$$
\begin{gathered}
\frac{x^{1} u^{\prime \prime}\left(\delta+(P+y) x^{1}\right)\left(u^{\prime}\left(\delta+(P+y) x^{2}\right)-1\right)-x^{2} u^{\prime \prime}\left(\delta+(P+y) x^{2}\right)\left(u^{\prime}\left(\delta+(P+y) x^{1}\right)-1\right)}{\left(u^{\prime}\left(\delta+(P+y) x^{2}\right)-1\right)^{2}}<0 \\
-x^{1} \frac{u^{\prime \prime}\left(\delta+(P+y) x^{1}\right)}{u^{\prime}\left(\delta+(P+y) x^{1}\right)-1}>-x^{2} \frac{u^{\prime \prime}\left(\delta+(P+y) x^{2}\right)}{u^{\prime}\left(\delta+(P+y) x^{2}\right)-1}
\end{gathered}
$$

which holds as soon as $\kappa>0$ (cf. expression 36). 


\section{F Proof of Proposition 6}

\section{F.1 Quantities}

We derive equations 40 and 41 relative to $y_{l}, l=B, G$ in the vicinity of the certain equilibrium

$$
\begin{gathered}
\alpha^{1} u^{\prime \prime}\left(\delta+(P+y) x^{1}\right)\left(x^{1}\left(\frac{\partial P_{B}}{\partial y_{l}}+1_{l=B}\right)+(P+y) \frac{\partial x^{1}}{\partial y_{l}}\right)=\alpha^{2} u^{\prime \prime}\left(\delta+(P+y) x^{2}\right)\left(x^{2}\left(\frac{\partial P_{B}}{\partial y_{l}}+1_{l=B}\right)+(P+y) \frac{\partial x^{2}}{\partial y_{l}}\right) \\
\alpha^{1} u^{\prime \prime}\left(\delta+(P+y) x^{1}\right)\left(x^{1}\left(\frac{\partial P_{G}}{\partial y_{l}}+1_{l=G}\right)+(P+y) \frac{\partial x^{1}}{\partial y_{l}}+(P-K) \frac{\partial s^{1}}{\partial y_{l}}\right)= \\
\alpha^{2} u^{\prime \prime}\left(\delta+(P+y) x^{2}\right)\left(x^{2}\left(\frac{\partial P_{G}}{\partial y_{l}}+1_{l=G}\right)+(P+y) \frac{\partial x^{2}}{\partial y_{l}}+(P-K) \frac{\partial s^{2}}{\partial y_{l}}\right)
\end{gathered}
$$

We deduce using the definition 36 ) of $\kappa$ :

$$
\begin{array}{r}
\eta^{1}(P+y) \frac{\partial x^{1}}{\partial y_{l}}=-\eta^{2}(P+y) \frac{\partial x^{2}}{\partial y_{l}}=-\kappa\left(\frac{\partial P_{B}}{\partial y_{l}}+1_{l=B}\right) \\
\eta^{1}(P+y) \frac{\partial x^{1}}{\partial y_{l}}+\eta^{1}(P-K) \frac{\partial s^{1}}{\partial y_{l}}=-\eta^{2}(P+y) \frac{\partial x^{1}}{\partial y_{l}}-\eta^{2}(P-K) \frac{\partial s^{2}}{\partial y_{l}}=-\kappa\left(\frac{\partial P_{G}}{\partial y_{l}}+1_{l=G}\right)
\end{array}
$$

\section{F.2 Prices}

We derive the equation 38 relative to $y_{l}$ :

$$
\begin{aligned}
\frac{\partial P_{k}}{\partial y_{l}} & =\beta\left(1+\alpha^{i}\left(u^{\prime}\left(\delta+(P+y) x^{i}\right)-1\right)\right) \sum_{j=B, G} \pi_{k, j}\left(\frac{\partial P_{j}}{\partial y_{l}}+1_{l=j}\right) \\
& +\alpha^{i} u^{\prime \prime}\left(\delta+(P+y) x^{i}\right)(P+y) \sum_{j=B, G} \pi_{k, j}\left(x^{i}+(-1)^{i} \frac{\kappa}{\eta^{i}}\right)\left(\frac{\partial P_{j}}{\partial y_{l}}+1_{l=j}\right)
\end{aligned}
$$

This is exactly the same expression as in the no-option economy.

$\left[\begin{array}{c}\frac{\partial P_{G}}{\partial y_{l}} \\ \frac{\partial P_{B}}{\partial y_{l}}\end{array}\right]=\frac{\beta \widehat{M}}{(1-\beta \widehat{M})\left(1-\left(\pi_{G G}+\pi_{B B}-1\right) \beta \widehat{M}\right)}\left[\begin{array}{c}\left(\pi_{G G}-\beta \widehat{M}\left(\pi_{G G}+\pi_{B B}-1\right)\right) 1_{l=G}+\left(1-\pi_{G G}\right) 1_{l=B} \\ \left(1-\pi_{B B}\right) 1_{l=G}+\left(\pi_{B B}-\beta \widehat{M}\left(\pi_{G G}+\pi_{B B}-1\right)\right) 1_{l=B}\end{array}\right]>0$

\section{F.3 Back to Proposition 6}

We are interested in the derivative of $x^{1}$ relative to $V[y]$ :

$$
\begin{aligned}
\eta^{1}(P+y) \frac{\partial x^{1}}{\partial V[y]}=-\eta^{2}(P+y) \frac{\partial x^{2}}{\partial V[y]} & =\frac{2-\pi_{G G}-\pi_{B B}}{2\left(y_{G}-y_{B}\right)} \eta^{1}(P+y)\left(\frac{1}{1-\pi_{B B}} \frac{\partial x^{1}}{\partial y_{G}}-\frac{1}{1-\pi_{G G}} \frac{\partial x^{1}}{\partial y_{B}}\right) \\
& =-\kappa \frac{\partial P_{B}}{\partial V[y]}-\kappa \frac{2-\pi_{G G}-\pi_{B B}}{2\left(y_{G}-y_{B}\right)}\left(-\frac{1}{1-\pi_{G G}}\right) \\
& =\kappa \frac{2-\pi_{G G}-\pi_{B B}}{2\left(y_{G}-y_{B}\right)}\left(-\frac{2\left(y_{G}-y_{B}\right)}{2-\pi_{G G}-\pi_{B B}} \frac{\partial P_{B}}{\partial V[y]}+\frac{1}{1-\pi_{G G}}\right) \\
& =\kappa \frac{2-\pi_{G G}-\pi_{B B}}{2\left(y_{G}-y_{B}\right)\left(1-\pi_{G G}\right)} \frac{1}{1-\left(\pi_{G G}+\pi_{B B}-1\right) \beta \widehat{M}}>0
\end{aligned}
$$


Indeed:

$$
\begin{aligned}
& \frac{2\left(y_{G}-y_{B}\right)}{2-\pi_{G G}-\pi_{B B}} \frac{\partial P_{G}}{\partial V[y]}=\frac{1}{1-\pi_{B B}} \frac{\left(\pi_{G G}+\pi_{B B}-1\right) \beta \widehat{M}}{1-\left(\pi_{G G}+\pi_{B B}-1\right) \beta \widehat{M}}>0 \\
& \frac{2\left(y_{G}-y_{B}\right)}{2-\pi_{G G}-\pi_{B B}} \frac{\partial P_{B}}{\partial V[y]}=-\frac{1}{1-\pi_{G G}} \frac{\left(\pi_{G G}+\pi_{B B}-1\right) \beta \widehat{M}}{1-\left(\pi_{G G}+\pi_{B B}-1\right) \beta \widehat{M}}<0
\end{aligned}
$$

We also have:

$$
\begin{aligned}
\eta^{1}(P+y) \frac{\partial x^{1}}{\partial V[y]}+\eta^{1}(P-K) \frac{\partial s^{1}}{\partial V[y]} & =-\eta^{2}(P+y) \frac{\partial x^{2}}{\partial V[y]}-\eta^{2}(P-K) \frac{\partial s^{2}}{\partial V[y]} \\
& =-\kappa \frac{\partial P_{G}}{\partial V[y]}-\kappa \frac{2-\pi_{G G}-\pi_{B B}}{2\left(y_{G}-y_{B}\right)}\left(\frac{1}{1-\pi_{B B}}\right) \\
& =-\kappa \frac{2-\pi_{G G}-\pi_{B B}}{2\left(y_{G}-y_{B}\right)\left(1-\pi_{B B}\right)} \frac{1}{1-\left(\pi_{G G}+\pi_{B B}-1\right) \beta \widehat{M}} \\
\eta^{1}(P-K) \frac{\partial s^{1}}{\partial V[y]} & =-\kappa \frac{2-\pi_{G G}-\pi_{B B}}{2\left(y_{G}-y_{B}\right)} \frac{1}{1-\left(\pi_{G G}+\pi_{B B}-1\right) \beta \widehat{M}} \frac{2-\pi_{B B}-\pi_{G G}}{\left(1-\pi_{B B}\right)\left(1-\pi_{G G}\right)}<0
\end{aligned}
$$

The last result to prove consists in focusing on the option price. Deriving the equation 39 relative to $y_{l}$ yields:

$$
\begin{aligned}
\frac{\partial Q_{k}}{\partial y_{l}} & =\beta \pi_{k, G}\left(1+\alpha^{i}\left(u^{\prime}\left(\delta+\left(P_{G}+y_{G}\right) x^{i}\right)-1\right)\right)\left(\frac{\partial P_{G}}{\partial y_{l}}+1_{l=G}\right) \\
& +\beta \pi_{k, G} \alpha^{i} u^{\prime \prime}\left(\delta+(P+y) x^{i}\right)(P-K)\left((P+y) \frac{\partial x^{i}}{\partial y_{l}}+(P-K) \frac{\partial s^{i}}{\partial y_{l}}+x^{i}\left(\frac{\partial P_{G}}{\partial y_{l}}+1_{l=G}\right)\right) \\
& =\beta \widehat{M}\left(\frac{\partial P_{G}}{\partial y_{l}}+1_{l=G}\right)
\end{aligned}
$$

We deduce:

$$
\frac{\partial Q_{k}}{\partial V[y]}=\beta \pi_{k, G} \widehat{M} \frac{\partial P_{G}}{\partial V[y]}+\beta \pi_{k, G} \widehat{M} \frac{2-\pi_{G G}-\pi_{B B}}{2\left(y_{G}-y_{B}\right)} \frac{1}{1-\pi_{B B}}=\pi_{k, G} \frac{2-\pi_{G G}-\pi_{B B}}{2\left(y_{G}-y_{B}\right)} \frac{1}{1-\pi_{B B}} \frac{\widehat{M}}{1-\left(\pi_{G G}+\pi_{B B}-1\right) \beta \widehat{M}}>0
$$

It is straightforward that $\frac{\partial Q_{G}}{\partial V[y]}>\frac{\partial Q_{B}}{\partial V[y]}$.

\section{G Proof of Proposition 7}

As in section $\mathrm{C}$ we study the evolution of prices and quantities around the certain equilibrium $\alpha^{1}=\alpha^{2}=\alpha$, where the asset prices are $P_{G}$ and $P_{B}$ and options one respectively $Q_{G}$ and $Q_{B}$. This equilibrium variables are defined as $(k=B, G)$ :

$$
\begin{aligned}
& P_{k}=\beta \sum_{j=B, G} \pi_{k, j}\left(1+\alpha\left(u^{\prime}\left(\delta+\left(P_{j}+y_{j}\right) \frac{V}{\eta^{1}+\eta^{2}}\right)-1\right)\right)\left(P_{j}+y_{j}\right) \\
& Q_{k}=\beta \pi_{k, G}\left(1+\alpha\left(u^{\prime}\left(\delta+\left(P_{G}+y_{G}\right) \frac{V}{\eta^{1}+\eta^{2}}\right)-1\right)\right)\left(P_{G}-K\right) \\
& \text { and: } \quad\left(\eta^{1}+\eta^{2}\right) x_{k}=V \text { and }\left(\eta^{1}+\eta^{2}\right) s_{k}=0
\end{aligned}
$$




\section{G.1 Quantities}

Deriving 40 and 41 yields:

$$
\begin{aligned}
&\left(1_{l=1}-1_{l=2}\right)\left(u^{\prime}\left(\delta+\left(P_{B}+y_{B}\right) \frac{V}{\eta^{1}+\eta^{2}}\right)-1\right)+\alpha u^{\prime \prime}\left(\delta+\left(P_{B}+y_{B}\right) \frac{V}{\eta^{1}+\eta^{2}}\right)\left(P_{B}+y_{B}\right) \frac{\partial x^{1}}{\partial \alpha^{l}}= \\
& \alpha u^{\prime \prime}\left(\delta+\left(P_{B}+y_{B}\right) \frac{V}{\eta^{1}+\eta^{2}}\right)\left(P_{B}+y_{B}\right) \frac{\partial x^{2}}{\partial \alpha^{l}} \\
& \eta^{1}\left(P_{B}+y_{B}\right) \frac{\partial x^{1}}{\partial \alpha^{l}}=\left(1_{l=2}-1_{l=1}\right) \frac{\eta^{1} \eta^{2}}{\eta^{1}+\eta^{2}} \frac{u^{\prime}\left(\delta+\left(P_{B}+y_{B}\right) \frac{V}{\eta^{1}+\eta^{2}}\right)-1}{\alpha u^{\prime \prime}\left(\delta+\left(P_{B}+y_{B}\right) \frac{V}{\eta^{1}+\eta^{2}}\right)}
\end{aligned}
$$

Analogously, we show that:

$$
\eta^{1}\left(P_{G}+y_{G}\right) \frac{\partial x^{1}}{\partial \alpha^{l}}+\eta^{1}\left(P_{G}-K\right) \frac{\partial s^{1}}{\partial \alpha^{l}}=\left(1_{l=2}-1_{l=1}\right) \frac{\eta^{1} \eta^{2}}{\eta^{1}+\eta^{2}} \frac{u^{\prime}\left(\delta+\left(P_{G}+y_{G}\right) \frac{V}{\eta^{1}+\eta^{2}}\right)-1}{\alpha u^{\prime \prime}\left(\delta+\left(P_{G}+y_{G}\right) \frac{V}{\eta^{1}+\eta^{2}}\right)}
$$

We deduce that:

- $\frac{\partial x^{1}}{\partial \alpha^{1}}>0$

- $\frac{\partial s^{1}}{\partial \alpha^{l}}<0$ iif $-\frac{u^{\prime \prime}\left(\delta+\left(P_{G}+y_{G}\right) \frac{V}{\eta^{1}+\eta^{2}}\right)}{u^{\prime}\left(\delta+\left(P_{G}+y_{G}\right) \frac{V}{\eta^{1}+\eta^{2}}\right)-1}>-\frac{u^{\prime \prime}\left(\delta+\left(P_{B}+y_{B}\right) \frac{V}{\eta^{1}+\eta^{2}}\right)}{u^{\prime}\left(\delta+\left(P_{B}+y_{B}\right) \frac{V}{\eta^{1}+\eta^{2}}\right)-1}$. 


\section{G.2 Prices}

We derive price expressions 38 and 39 relative to $\alpha^{l}, l=B, G$.

$$
\begin{aligned}
\frac{\partial P_{k}}{\partial \alpha^{l}} & =\beta \sum_{j=B, G} \pi_{k, j}\left(1+\alpha\left(u^{\prime}\left(\delta+\left(P_{j}+y_{j}\right) \frac{V}{\eta^{1}+\eta^{2}}\right)-1\right)\right) \frac{\partial P_{j}}{\partial \alpha^{l}} \\
& +\beta \sum_{j=B, G} \pi_{k, j} \alpha u^{\prime \prime}\left(\delta+\left(P_{j}+y_{j}\right) \frac{V}{\eta^{1}+\eta^{2}}\right)\left(P_{j}+y_{j}\right)\left(\frac{V}{\eta^{1}+\eta^{2}} \frac{\partial P_{j}}{\partial \alpha^{l}}+\left(P_{j}+y_{j}\right) \frac{\partial x^{i}}{\partial \alpha^{l}}+1_{j=G}\left(P_{G}-K\right) \frac{\partial s^{i}}{\partial \alpha^{l}}\right) \\
& +1_{l=i} \beta \sum_{j=B, G} \pi_{k, j}\left(u^{\prime}\left(\delta+\left(P_{j}+y_{j}\right) \frac{V}{\eta^{1}+\eta^{2}}\right)-1\right)\left(P_{j}+y_{j}\right) \\
& =\beta \sum_{j=B, G} \pi_{k, j}\left(1+\alpha\left(u^{\prime}\left(\delta+\left(P_{j}+y_{j}\right) \frac{V}{\eta^{1}+\eta^{2}}\right)-1\right)\left(1+\frac{\left(P_{j}+y_{j}\right) \frac{V}{\eta^{1}+\eta^{2}} u^{\prime \prime}\left(\delta+\left(P_{j}+y_{j}\right) \frac{V}{\eta^{1}+\eta^{2}}\right)}{u^{\prime}\left(\delta+\left(P_{j}+y_{j}\right) \frac{V}{\eta^{1}+\eta^{2}}\right)-1}\right)\right) \frac{\partial P_{j}}{\partial \alpha^{l}} \\
& +\beta\left(1_{l=i}+\frac{(-1)^{i}}{\eta^{i}}\left(1_{l=1}-1_{l=2}\right) \frac{\eta^{1} \eta^{2}}{\eta^{1}+\eta^{2}}\right) \sum_{j=B, G} \pi_{k, j}\left(u^{\prime}\left(\delta+\left(P_{j}+y_{j}\right) \frac{V}{\eta^{1}+\eta^{2}}\right)-1\right)\left(P_{j}+y_{j}\right) \\
& =\beta \frac{\eta^{1} 1_{l=1}+\eta^{2} 1_{l=2}}{\eta^{1}+\eta^{2}} \sum_{j=B, G} \pi_{k, j} \Delta_{j}+\beta \sum_{j=B, G} \pi_{k, j} M_{j} \frac{\partial P_{j}}{\partial \alpha^{l}}
\end{aligned}
$$

with: $M_{j}=1+\alpha\left(u^{\prime}\left(\delta+\left(P_{j}+y_{j}\right) \frac{V}{\eta^{1}+\eta^{2}}\right)-1\right)\left(1+\frac{\left(P_{j}+y_{j}\right) V}{\eta^{1}+\eta^{2}} \frac{u^{\prime \prime}\left(\delta+\left(P_{j}+y_{j}\right) \frac{V}{\eta^{1}+\eta^{2}}\right)}{u^{\prime}\left(\delta+\frac{\left(P_{j}+y_{j}\right) V}{\eta^{1}+\eta^{2}}\right)-1}\right) \quad j=B, G$

$$
\begin{aligned}
\Delta_{j} & =\left(u^{\prime}\left(\delta+\left(P_{j}+y_{j}\right) \frac{V}{\eta^{1}+\eta^{2}}\right)-1\right)\left(P_{j}+y_{j}\right) \\
& +1_{l=i} \beta \sum_{j=B, G} \pi_{k, j}\left(u^{\prime}\left(\delta+\left(P_{j}+y_{j}\right) \frac{V}{\eta^{1}+\eta^{2}}\right)-1\right)\left(P_{j}+y_{j}\right) \\
& \beta \pi_{k, G}\left(1+\alpha^{i}\left(u^{\prime}\left(\delta+\left(P_{G}+y_{G}\right) x_{k}^{i}+\left(P_{G}-K\right) s_{k}^{i}\right)-1\right)\right)\left(P_{G}+y_{G}\right) \\
& +\beta \pi_{k, B}\left(1+\alpha^{i}\left(u^{\prime}\left(\delta+\left(P_{B}+y_{B}\right) x_{k}^{i}\right)-1\right)\right)\left(P_{B}+y_{B}\right) \\
Q_{k} & =\beta \pi_{k, G}\left(1+\left(1-\alpha^{i}\right)\left(u^{\prime}\left(\delta+\left(P_{G}+y_{G}\right) x_{k}^{i}+\left(P_{G}-K\right) s_{k}^{i}\right)-1\right)\right)\left(P_{G}-K\right)
\end{aligned}
$$

The computation is very similar to the no-option economy and we find identically:

$$
\begin{aligned}
\left(1-\beta \pi_{G G} M_{G}-\beta \pi_{B B} M_{B}+\beta^{2}\left(\pi_{G G}+\pi_{B B}-1\right) M_{G} M_{B}\right)\left[\begin{array}{c}
\frac{\partial P_{G}}{\partial \alpha^{l}} \\
\frac{\partial P_{B}}{\partial \alpha^{l}}
\end{array}\right]= \\
\beta \frac{\eta^{1} 1_{l=1}+\eta^{2} 1_{l=2}}{\eta^{1}+\eta^{2}}\left[\begin{array}{c}
\left(\pi_{G G}-\beta M_{B}\left(\pi_{G G}+\pi_{B B}-1\right)\right) \Delta_{G}+\left(1-\pi_{G G}\right) \Delta_{B} \\
\left(\pi_{B B}-\beta M_{G}\left(\pi_{G G}+\pi_{B B}-1\right)\right) \Delta_{B}+\left(1-\pi_{B B}\right) \Delta_{G}
\end{array}\right]>0
\end{aligned}
$$


Regarding the option price, we have:

$$
\begin{aligned}
\frac{\partial Q_{k}}{\partial \alpha^{l}} & =\beta \pi_{k, G}\left(1+\alpha\left(u^{\prime}\left(\delta+\left(P_{G}+y_{G}\right) \frac{V}{\eta^{1}+\eta^{2}}\right)-1\right)\right) \frac{\partial P_{G}}{\partial \alpha^{l}} \\
& +\beta \pi_{k, G} \alpha u^{\prime \prime}\left(\delta+\left(P_{G}+y_{G}\right) \frac{V}{\eta^{1}+\eta^{2}}\right)\left(P_{G}-K\right)\left(\frac{V}{\eta^{1}+\eta^{2}} \frac{\partial P_{G}}{\partial \alpha^{l}}+\left(P_{G}+y_{G}\right) \frac{\partial x^{i}}{\partial \alpha^{l}}+\left(P_{G}-K\right) \frac{\partial s^{i}}{\partial \alpha^{l}}\right) \\
& +1_{l=i} \beta \pi_{k, G}\left(u^{\prime}\left(\delta+\left(P_{G}+y_{G}\right) \frac{V}{\eta^{1}+\eta^{2}}\right)-1\right)\left(P_{G}-K\right) \\
& =\beta \pi_{k, G}\left(1+\alpha\left(u^{\prime}\left(\delta+\left(P_{G}+y_{G}\right) \frac{V}{\eta^{1}+\eta^{2}}\right)-1\right)\left(1+\frac{\left(P_{G}-K\right) \frac{V}{\eta^{1}+\eta^{2}} u^{\prime \prime}\left(\delta+\left(P_{G}+y_{G}\right) \frac{V}{\eta^{1}+\eta^{2}}\right)}{u^{\prime}\left(\delta+\left(P_{G}+y_{G}\right) \frac{V}{\eta^{1}+\eta^{2}}\right)-1}\right)\right) \frac{\partial P_{G}}{\partial \alpha^{l}} \\
& +\beta\left(1_{l=i}+\frac{(-1)^{i}}{\eta^{i}}\left(1_{l=1}-1_{l=2}\right) \frac{\eta^{1} \eta^{2}}{\eta^{1}+\eta^{2}}\right) \pi_{k, G}\left(u^{\prime}\left(\delta+\left(P_{G}+y_{G}\right) \frac{V}{\eta^{1}+\eta^{2}}\right)-1\right)\left(P_{G}-K\right) \\
& =\beta \pi_{k, G}\left(1+\alpha\left(u^{\prime}\left(\delta+\left(P_{G}+y_{G}\right) \frac{V}{\eta^{1}+\eta^{2}}\right)-1\right)\left(1+\frac{\left(P_{G}-K\right) \frac{V}{\eta^{1}+\eta^{2}} u^{\prime \prime}\left(\delta+\left(P_{G}+y_{G}\right) \frac{V}{\eta^{1}+\eta^{2}}\right)}{u^{\prime}\left(\delta+\left(P_{G}+y_{G}\right) \frac{V}{\eta^{1}+\eta^{2}}\right)-1}\right)\right) \frac{\partial P_{G}}{\partial \alpha^{l}}
\end{aligned}
$$

We easily deduce that $\frac{\partial Q_{G}}{\partial \alpha^{l}}>\frac{\partial Q_{B}}{\partial \alpha^{l}}>0$, which proves the last result of Proposition 7

\section{H Proof of Proposition 8}

We now turn to the impact of option introduction on the welfare.

\section{H.1 General case}

In order to derive welfares, we need expressions for consumption and labor. For example $c_{k, j}^{u e, i}$ is the consumption of an agent ue of type $i=1,2$ who was in the previous period in state $k$ and who is currently in the state $j=B, G$.

$$
\begin{aligned}
c_{k, j}^{e e, i} & =c_{k, j}^{u e, i}=u^{\prime-1}(1) \\
c_{k, j}^{e u, i} & =\delta+\left(P_{j}+y_{j}\right) x_{k}^{i}+\left(P_{j}-K\right)^{+} s_{k}^{i} \\
c_{k, j}^{u u, i} & =\delta
\end{aligned}
$$

Using budget constraints, we obtain:

$$
\begin{aligned}
& l_{k, j}^{e e, i}=c_{k, j}^{e e, i}+Q_{j} s_{j}^{i}-\left(P_{j}-K\right)^{+} s_{k}^{i}+P_{j} x_{j}^{i}-\left(P_{j}+y_{j}\right) x_{k}^{i} \\
& l_{k, j}^{u e, i}=c_{k, j}^{u e, i}+Q_{j} s_{j}^{i}+P_{j} x_{j}^{i} \\
& l_{k, j}^{u u, i}=l_{k, j}^{e u, i}=0
\end{aligned}
$$

We deduce the instantaneous utilities that we note $u_{k, j}^{\star, i}$, where $\star$ is the agent's class, $i$ his type, and $k, l=B, G$ 
aggregate states:

$$
\begin{aligned}
u_{k, j}^{e e, i} & =u\left(u^{\prime-1}(1)\right)-u^{\prime-1}(1)-Q_{j} s_{j}^{i}+\left(P_{j}-K\right)^{+} s_{k}^{i}+\left(P_{j}+y_{j}\right) x_{k}^{i}-P_{j} x_{j}^{i} \\
u_{k, j}^{u e, i} & =u\left(u^{\prime-1}(1)\right)-u^{\prime-1}(1)-Q_{j} s_{j}^{i}-P_{j} x_{j}^{i} \\
u_{k, j}^{e u, i} & =u\left(\delta+\left(P_{j}+y_{j}\right) x_{k}^{i}+\left(P_{j}-K\right)^{+} s_{k}^{i}\right) \\
u_{k, j}^{u u, i} & =u(\delta)
\end{aligned}
$$

Noting $u=u\left(u^{\prime-1}(1)\right)-u^{\prime-1}(1)$, the instantaneous utility vector $\mathcal{U}^{i}$ of an agent $i$ expresses as $\mathcal{U}^{i}=$ $\left[u_{G, G}^{e e, i}, u_{B, G}^{e e, i}, u_{G, B}^{e e, i}, u_{B, B}^{e e, i}, u_{G, G}^{u e, i}, \ldots, u_{G, G}^{e u, i}, \ldots, u_{G, G}^{u u, i}, \ldots, u_{B, B}^{u u, i}\right]^{\top}$ or:

$$
\mathcal{U}^{i}=\left[\begin{array}{c}
u-\left(Q_{G}-\left(P_{G}-K\right)\right) s_{G}^{i}+y_{G} x_{G}^{i} \\
u-\left(Q_{G} s_{G}^{i}-\left(P_{G}-K\right) s_{B}^{i}\right)+\left(P_{G}+y_{G}\right) x_{B}^{i}-P_{G} x_{G}^{i} \\
u-Q_{B} s_{B}^{i}+\left(P_{B}+y_{B}\right) x_{G}^{i}-P_{B} x_{B}^{i} \\
u-Q_{B} s_{B}^{i}+y_{B} x_{B}^{i} \\
u-Q_{G} s_{G}^{i}-P_{G} x_{G}^{i} \\
u-Q_{G} s_{G}^{i}-P_{G} x_{G}^{i} \\
u-Q_{B} s_{B}^{i}-P_{B} x_{B}^{i} \\
u-Q_{B} s_{B}^{i}-P_{B} x_{B}^{i} \\
u\left(\delta+\left(P_{G}+y_{G}\right) x_{G}^{i}+\left(P_{G}-K\right) s_{G}^{i}\right) \\
u\left(\delta+\left(P_{G}+y_{G}\right) x_{B}^{i}+\left(P_{G}-K\right) s_{B}^{i}\right) \\
u\left(\delta+\left(P_{B}+y_{B}\right) x_{G}^{i}\right) \\
u\left(\delta+\left(P_{B}+y_{B}\right) x_{B}^{i}\right) \\
u(\delta) 1_{4}
\end{array}\right]
$$

In order to compute intertemporal utility, we need the expression of the transition matrix $M^{i}$, depending on 
the agent's type. The matrix express as $M^{i}=\Omega^{i} \otimes \Lambda$, with:

$$
\Omega^{i}=\left[\begin{array}{cccc}
\alpha^{i} & 0 & 1-\alpha^{i} & 0 \\
\alpha^{i} & 0 & 1-\alpha^{i} & 0 \\
0 & 1-\rho^{i} & 0 & \rho^{i} \\
0 & 1-\rho^{i} & 0 & \rho^{i}
\end{array}\right]=P^{i} D^{i}\left(P^{i}\right)^{-1}, \text { with } P^{i}=\left[\begin{array}{cccc}
1 & 1-\alpha^{i} & 0 & 1-\alpha^{i} \\
1 & 0 & \rho^{i} & 1-\alpha^{i} \\
1 & -\alpha^{i} & 0 & -\left(1-\rho^{i}\right) \\
1 & 0 & -\left(1-\rho^{i}\right) & -\left(1-\rho^{i}\right)
\end{array}\right]
$$

and $D^{i}=\operatorname{Diag}\left(\begin{array}{llll}1 & 0 & 0 & \alpha^{i}+\rho^{i}-1\end{array}\right)$

$$
\Lambda=\left[\begin{array}{cccc}
\pi_{G G} & 0 & 1-\pi_{G G} & 0 \\
\pi_{G G} & 0 & 1-\pi_{G G} & 0 \\
0 & 1-\pi_{B B} & 0 & \pi_{B B} \\
0 & 1-\pi_{B B} & 0 & \pi_{B B}
\end{array}\right]=Q \Delta Q^{-1}, \text { with } Q=\left[\begin{array}{cccc}
1 & 1-\pi_{G G} & 0 & 1-\pi_{G G} \\
1 & 0 & \pi_{B B} & 1-\pi_{G G} \\
1 & -\pi_{G G} & 0 & -\left(1-\pi_{B B}\right) \\
1 & 0 & -\left(1-\pi_{B B}\right) & -\left(1-\pi_{B B}\right)
\end{array}\right]
$$

and $\Delta=\operatorname{Diag}\left(\begin{array}{llll}1 & 0 & 0 & \left.\pi_{G G}+\pi_{B B}-1\right)\end{array}\right.$

$$
\begin{aligned}
& \left(P^{i}\right)^{-1}=\left[\begin{array}{cccc}
\frac{\alpha^{i}\left(1-\rho^{i}\right)}{2-\alpha^{i}-\rho^{i}} & \frac{\left(1-\alpha^{i}\right)\left(1-\rho^{i}\right)}{2-\alpha^{i}-\rho^{i}} & \frac{\left(1-\alpha^{i}\right)\left(1-\rho^{i}\right)}{2-\alpha^{i}-\rho^{i}} & \frac{\left(1-\alpha^{i}\right) \rho^{i}}{2-\alpha^{i}-\rho^{i}} \\
\frac{-\left(1-\rho^{i}\right)}{\alpha^{i}+\rho^{i}-1} & \frac{\left(1-\rho^{i}\right)}{\alpha^{i}+\rho^{i}-1} & \frac{\rho^{i}}{\alpha^{i}+\rho^{i}-1} & \frac{\rho^{i}}{\alpha^{i}+\rho^{i}-1} \\
\frac{-\alpha^{i}}{\alpha^{i}+\rho^{i}-1} & \frac{\alpha^{i}}{\alpha^{i}+\rho^{i}-1} & \frac{-\left(1-\alpha^{i}\right)}{\alpha^{i}+\rho^{i}-1} & \frac{\left(1-\alpha^{i}\right)}{\alpha^{i}+\rho^{i}-1} \\
\frac{\alpha^{i}}{\left(\alpha^{i}+\rho^{i}-1\right)\left(2-\alpha^{i}-\rho^{i}\right)} & \frac{-\left(1-\rho^{i}\right)}{\left(\alpha^{i}+\rho^{i}-1\right)\left(2-\alpha^{i}-\rho^{i}\right)} & \frac{\left(1-\alpha^{i}\right)}{\left(\alpha^{i}+\rho^{i}-1\right)\left(2-\alpha^{i}-\rho^{i}\right)} & \frac{-\rho^{i}}{\left(\alpha^{i}+\rho^{i}-1\right)\left(2-\alpha^{i}-\rho^{i}\right)}
\end{array}\right]
\end{aligned}
$$

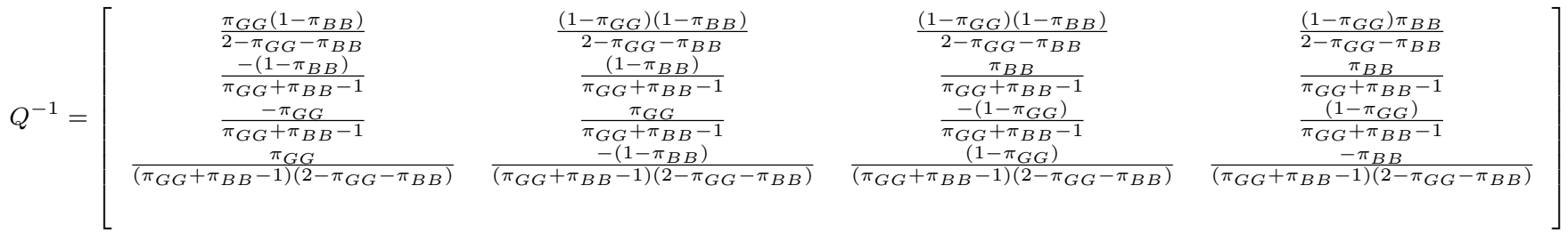

The ex-ante welfare $U^{i}$ of an agent of type $i$ is equal to the intertemporal sum of instantaneous utilities:

$$
\begin{aligned}
U^{i} & =\sum_{k=0}^{\infty} \beta^{k}\left(M^{i}\right)^{k} \mathcal{U}^{i}=\left[\sum_{k=0}^{\infty} \beta^{k}\left(\Omega^{i} \otimes T\right)^{k}\right] \mathcal{U}^{i}=\left[\sum_{k=0}^{\infty} \beta^{k}\left(\Omega^{i}\right)^{k} \otimes T^{k}\right] \mathcal{U}^{i}=\left[\sum_{k=0}^{\infty} \beta^{k}\left(P^{i}\left(D^{i}\right)^{k}\left(P^{i}\right)^{-1}\right) \otimes\left(Q \Delta^{k} Q^{-1}\right)\right] \mathcal{U}^{i} \\
& =\left[\sum_{k=0}^{\infty} \beta^{k}\left(P^{i} \otimes Q\right)\left(\left(D^{i}\right)^{k} \otimes \Delta^{k}\right)\left(\left(P^{i}\right)^{-1} \otimes Q^{-1}\right)\right] \mathcal{U}^{i}=\left(P^{i} \otimes Q\right)\left[\sum_{k=0}^{\infty} \beta^{k}\left(\left(D^{i}\right)^{k} \otimes \Delta^{k}\right)\right]\left(\left(P^{i}\right)^{-1} \otimes Q^{-1}\right) \mathcal{U}^{i}
\end{aligned}
$$

We denote $U_{a}^{i}$ and $U_{o}^{i}$ the ex-ante welfares in the economy respectively without and with option. Analogously $\mathcal{U}_{a}^{i}$ and $\mathcal{U}_{o}^{i}$ are instantaneous utility expressions in economies without and with option.

We compute the derivatives $\frac{\partial \mathcal{U}_{o}^{i}}{\partial y_{1}}$ and $\frac{\partial \mathcal{U}_{a}^{i}}{\partial y_{1}}$ in the vicinity of the equilibrium without uncertainty, where the option is not traded and both welfares equal to each other. 


\section{H.2 In the economy with an option}

The utility vector expression 44 , as well as the variation of $\mathcal{U}_{a}^{1}$ relative to $y_{1}$, express without option $\left(s_{k}^{i}=0\right)$ :

$$
\mathcal{U}_{a}^{i}=\left[\begin{array}{c}
u+y_{G} x_{G}^{i} \\
u-P_{G}\left(x_{G}^{i}-x_{B}^{i}\right)+y_{G} x_{B}^{i} \\
u-P_{B}\left(x_{B}^{i}-x_{G}^{i}\right)+y_{B} x_{G}^{i} \\
u+y_{B} x_{B}^{i} \\
u-P_{G} x_{G}^{i} \\
u-P_{G} x_{G}^{i} \\
u-P_{B} x_{B}^{i} \\
u-P_{B} x_{B}^{i} \\
u\left(\delta+\left(P_{G}+y_{G}\right) x_{G}^{i}\right) \\
u\left(\delta+\left(P_{G}+y_{G}\right) x_{B}^{i}\right) \\
u\left(\delta+\left(P_{B}+y_{B}\right) x_{G}^{i}\right) \\
u\left(\delta+\left(P_{B}+y_{B}\right) x_{B}^{i}\right) \\
u(\delta) 1_{4}
\end{array}\right]
$$

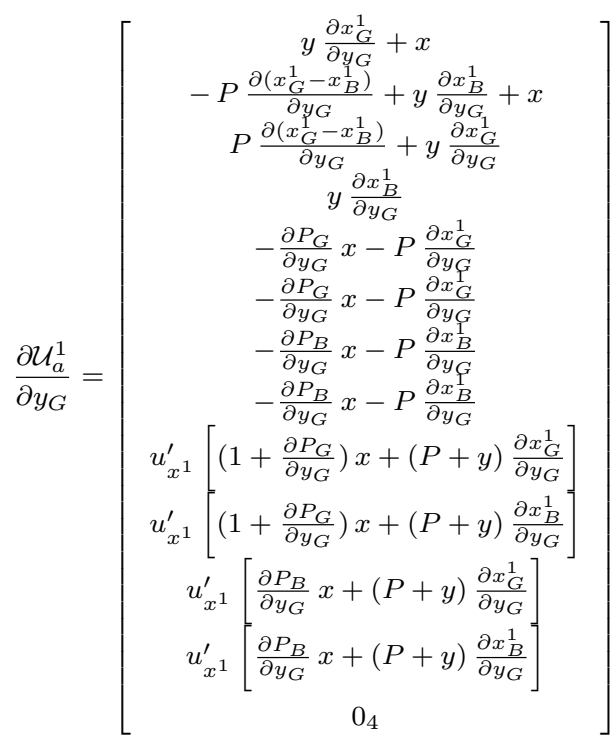

\section{H.3 In the economy without option}

Since portfolios are the same in both states of the world, we have $s_{G}^{i}=s_{B}^{i}=s^{i}$ and $x_{G}^{i}=x_{B}^{i}=x^{i}$ and we deduce:

$\mathcal{U}_{o}^{i}=\left[\begin{array}{c}u-\left(Q_{G}-\left(P_{G}-K\right)\right) s^{i}+y_{G} x^{i} \\ u-Q_{B} s^{i}+y_{B} x^{i} \\ u-Q_{G} s^{i}-P_{G} x^{i} \\ u-Q_{B} s^{i}-P_{B} x^{i} \\ u\left(\delta+\left(P_{G}+y_{G}\right) x^{i}+\left(P_{G}-K\right) s^{i}\right) \\ u\left(\delta+\left(P_{B}+y_{B}\right) x^{i}\right) \\ u(\delta) 1_{2}\end{array}\right] \otimes 1_{2} \frac{\partial \mathcal{U}_{o}^{1}}{\partial y_{G}}=\left[\begin{array}{c}-(Q-(P-K)) \frac{\partial s^{1}}{\partial y_{G}}+x+y \frac{\partial x^{1}}{\partial y_{G}} \\ -Q \frac{\partial s^{1}}{\partial y_{G}}+y \frac{\partial x^{1}}{\partial y_{G}} \\ -Q \frac{\partial s^{1}}{\partial y_{G}}-P \frac{\partial x^{1}}{\partial y_{G}}-\frac{\partial P_{G}}{\partial y_{G}} x \\ -Q \frac{\partial s^{1}}{\partial y_{G}}-P \frac{\partial x^{1}}{\partial y_{G}}-\frac{\partial P_{B}}{\partial y_{G}} x \\ u_{x^{1}}^{\prime}\left[\left(1+\frac{\partial P_{G}}{\partial y_{G}}\right) x+(P+y) \frac{\partial x^{1}}{\partial y_{G}}+(P-K) \frac{\partial s^{1}}{\partial y_{G}}\right] \\ u_{x^{1}}^{\prime}\left[\frac{\partial P_{B}}{\partial y_{G}} x+(P+y) \frac{\partial x^{1}}{\partial y_{G}}\right] \\ 0_{2}\end{array}\right] \otimes 1_{2}$ 


\section{H.3.1 Effects of the option introduction}

We compute the difference between both economies (without and with the option):

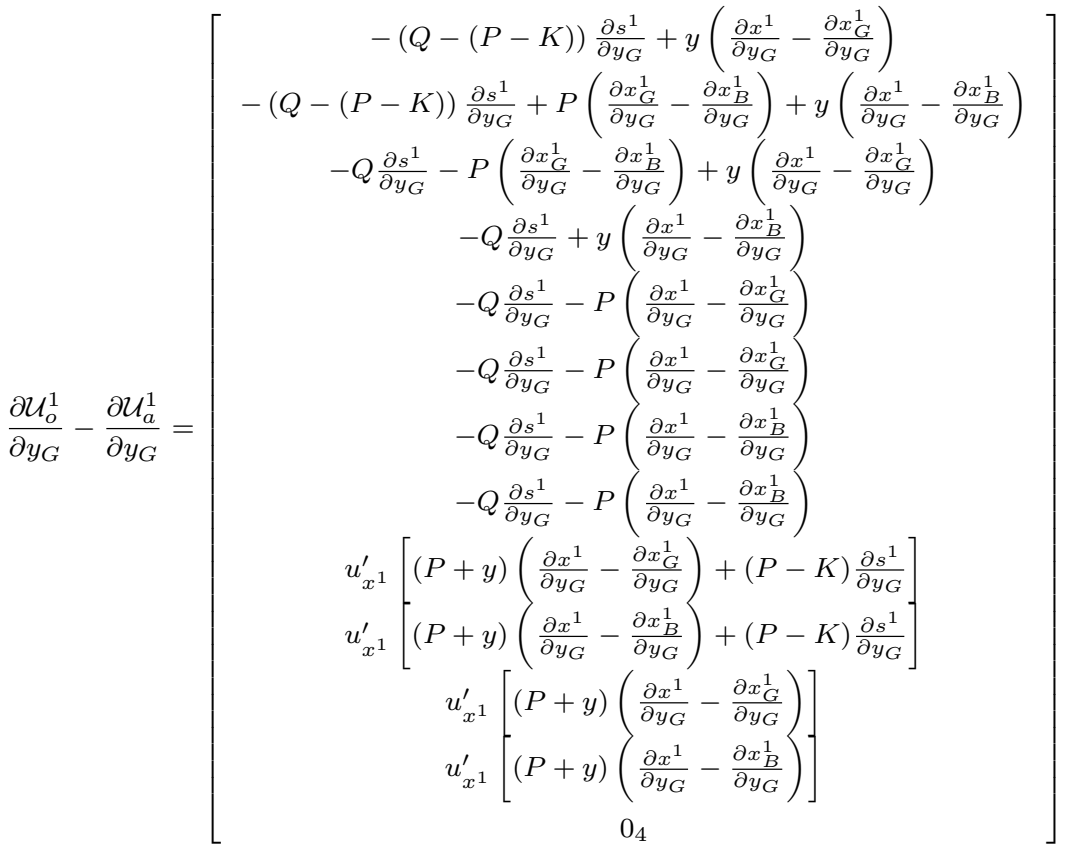

Some remarks:

- Welfare variations only depend on quantities variations. The price variations in particular do not influence welfare variations.

- Since holding variations of the agent 2 are the opposite of the ones of agent 1 , the welfare impact of options will opposite for both agents: one will lose and one will win.

Before going further in the computations of welfare, let us make the following preliminary computations:

$$
\begin{aligned}
\eta^{1}(P+y)\left(\frac{\partial x^{1}}{\partial y_{G}}-\frac{\partial x_{G}^{1}}{\partial y_{G}}\right) & =\frac{\kappa}{1-\left(\pi_{G G}+\pi_{B B}-1\right) \beta \widehat{M}} \pi_{G G} \\
\eta^{1}(P+y)\left(\frac{\partial x^{1}}{\partial y_{G}}-\frac{\partial x_{B}^{1}}{\partial y_{G}}\right) & =\frac{\kappa}{1-\left(\pi_{G G}+\pi_{B B}-1\right) \beta \widehat{M}}\left(1-\pi_{B B}\right) \\
\eta^{1}(P+y)\left(\frac{\partial x_{G}^{1}}{\partial y_{G}}-\frac{\partial x_{B}^{1}}{\partial y_{G}}\right) & =-\frac{\kappa}{1-\left(\pi_{G G}+\pi_{B B}-1\right) \beta \widehat{M}}\left(\pi_{G G}+\pi_{B B}-1\right) \\
\eta^{1}(P-K) \frac{\partial s^{1}}{\partial y_{G}} & =-\frac{\kappa}{1-\left(\pi_{G G}+\pi_{B B}-1\right) \beta \widehat{M}}
\end{aligned}
$$

Using pricing equations, we also define $0<\gamma<1$ as follows:

$$
\begin{aligned}
\gamma & =\frac{P}{P+y}=\frac{Q}{P-K}=\beta\left(1+\alpha^{i}\left(u^{\prime}\left(\delta+(P+y) x^{i}\right)-1\right)\right) \\
1-\gamma & =\frac{y}{P+y}=1-\beta\left(1+\alpha^{i}\left(u^{\prime}\left(\delta+(P+y) x^{i}\right)-1\right)\right)
\end{aligned}
$$


Since $\alpha^{1}\left(u^{\prime}\left(\delta+(P+y) x^{1}\right)-1\right)=\alpha^{2}\left(u^{\prime}\left(\delta+(P+y) x^{2}\right)-1\right), \gamma$ does not depend on the type $i$.

We therefore obtain that:

$$
\eta^{1} \frac{\partial \mathcal{U}_{o}^{1}}{\partial y_{G}}-\eta^{1} \frac{\partial \mathcal{U}_{a}^{1}}{\partial y_{G}}=-\left(\eta^{2} \frac{\partial \mathcal{U}_{o}^{2}}{\partial y_{G}}-\eta^{2} \frac{\partial \mathcal{U}_{a}^{2}}{\partial y_{G}}\right)=\frac{\kappa}{1-\left(\pi_{G G}+\pi_{B B}-1\right) \beta \widehat{M}}\left[\begin{array}{c}
-(1-\gamma)\left(1-\pi_{G G}\right) \\
-(1-\gamma) \pi_{B B}-\gamma\left(\pi_{G G}+\pi_{B B}-1\right) \\
\gamma\left(\pi_{G G}+\pi_{B B}\right)+(1-\gamma) \pi_{G G} \\
\gamma+(1-\gamma)\left(1-\pi_{B B}\right) \\
\gamma\left(1-\pi_{G G}\right) \\
\gamma\left(1-\pi_{G G}\right) \\
\gamma \pi_{B B} \\
\gamma \pi_{B B} \\
-u_{x^{1}}^{\prime}\left(1-\pi_{G G}\right) \\
-u_{x^{1}}^{\prime} \pi_{B B} \\
u_{x^{1}}^{\prime} \pi_{G G} \\
u_{x^{1}}^{\prime}\left(1-\pi_{B B}\right) \\
0
\end{array}\right]
$$

To compute the ex ante utility, we multiply that by the vector of weights, which is equal to:

$$
\begin{aligned}
W^{i}= & {\left[\frac{\alpha^{i}\left(1-\rho^{i}\right)}{2-\alpha^{i}-\rho^{i}}, \frac{\left(1-\alpha^{i}\right)\left(1-\rho^{i}\right)}{2-\alpha^{i}-\rho^{i}}, \frac{\left(1-\alpha^{i}\right)\left(1-\rho^{i}\right)}{2-\alpha^{i}-\rho^{i}}, \frac{\left(1-\alpha^{i}\right) \rho^{i}}{2-\alpha^{i}-\rho^{i}}\right] } \\
& \otimes\left[\frac{\pi_{G G}\left(1-\pi_{B B}\right)}{2-\pi_{G G}-\pi_{B B}}, \frac{\left(1-\pi_{G G}\right)\left(1-\pi_{B B}\right)}{2-\pi_{G G}-\pi_{B B}}, \frac{\left(1-\pi_{G G}\right)\left(1-\pi_{B B}\right)}{2-\pi_{G G}-\pi_{B B}}, \frac{\left(1-\pi_{G G}\right) \pi_{B B}}{2-\pi_{G G}-\pi_{B B}}\right]
\end{aligned}
$$

The difference of variations in ex ante utilities $\frac{\partial U_{o}^{i}}{\partial y_{G}}-\frac{\partial U_{a}^{i}}{\partial y_{G}}$ for the agent $i=1,2$ is equal to:

$$
\frac{\partial U_{o}^{1}}{\partial y_{G}}-\frac{\partial U_{a}^{1}}{\partial y_{G}}=-\left(\frac{\partial U_{o}^{2}}{\partial y_{G}}-\frac{\partial U_{a}^{2}}{\partial y_{G}}\right)=\frac{\kappa}{\left(1-\left(\pi_{G G}+\pi_{B B}-1\right) \widehat{\beta}\right)} \frac{\left(1-\pi_{G G}\right) \gamma}{(1-\beta)\left(2-\pi_{G G}-\pi_{B B}\right)}>0
$$

The aggregate ex ante welfare is unchanged (if we suppose that being of type 1 or 2 is equiprobable). However, type 1 agents facing a larger unemployment risk benefit from the option introduction, while the other type suffers from it.

\section{Proof of Proposition 9}

We use a proof strategy very similar to Section A We define $X=\left(y_{1}, \ldots, y_{n}, V, \alpha^{1}, \ldots, \alpha^{I}, w_{1}^{1}, \ldots, w_{n}^{1}, \ldots, w_{1}^{I}, \ldots, w_{n}^{I}\right) \in$ $\left(\mathbb{R}^{+}\right)^{n+1+I+n I}$ the vector of parameters and $Z=\left(P_{1}, \ldots, P_{n}, x_{1}^{1}, \ldots, x_{n}^{1}, \ldots, x_{1}^{I}, x_{n}^{I}\right) \in\left(\mathbb{R}^{+}\right)^{n+n I}$ the vector of endogenous variables.

We define the function $F:\left(\mathbb{R}^{+}\right)^{n+1+I+n I} \times\left(\mathbb{R}^{+}\right)^{n+n I} \longrightarrow\left(\mathbb{R}^{+}\right)^{n+n I}$ as follows $(i=1, \ldots, I$ and $k=1, \ldots, n)$ :

$$
\begin{aligned}
F_{(i-1) n+k}(X, Z) & =\frac{P_{k}}{w_{k}^{i}}-\beta \sum_{j=1}^{n} \pi_{k, j}\left(\frac{1-\alpha^{i}}{w_{j}^{i}}+\alpha^{i} u^{\prime}\left(\left(P_{j}+y_{j}\right) x_{k}^{i}+\delta\right)\right)\left(P_{j}+y_{j}\right) \\
F_{n I+k}(X, Z) & =\sum_{i=1}^{I} \eta^{i} x_{k}^{i}-V
\end{aligned}
$$


$F$ stacks the functions concerning each agents and the market equilibrium. The first $n$ equations are the Euler equations of agents of type 1 in the $n$ states of the world, the second set of $n$ equations concern agents of type 2 , and so on and so forth. The last set of $n$ equations is the set of market equilibria in the $n$ states of the world.

For a given set of parameters $X$, the equilibrium is defined as

$$
F(X, Z)=0
$$

Symmetric equilibrium. We already know that an equilibrium $\left(X^{*}, Z^{*}\right)$ exists for $X^{*}=\left(y, \ldots, y, V^{*}, \alpha, \ldots, \alpha, 1, \ldots, 1\right) \in$ $\left(\mathbb{R}^{+}\right)^{n+1+I+n I}$. The equilibrium is such that $Y^{*}=(P, \ldots, P, x, \ldots, x)$.

Implicit function theorem. To show that the equilibrium exists around $X^{\star}$, we show that the Jacobian $J_{F}$ of $F$ relative to $Z$ is invertible in $\left(X^{*}, Z^{*}\right)$

$$
J_{F}=\left(\frac{\partial F_{i}}{\partial y_{j}}\left(X^{*}, Z^{*}\right)\right)_{i=1, \ldots,(n+1) I, j=1, \ldots,(n+1) I}
$$

- Derivatives of $F$ relative to $P_{l}(i=1, \ldots, I$, and $k, l=1, \ldots, n)$ :

$$
\begin{aligned}
\frac{\partial F_{(i-1) n+k}}{\partial P_{j}} & =1-\beta \pi_{k, j}\left(1-\alpha+\alpha u^{\prime}(\delta+(P+y) x)\right) \\
& -\beta \pi_{k, j} \alpha x u^{\prime \prime}(\delta+(P+y) x)(P+y) \\
\frac{\partial F_{n L+k}}{\partial P_{j}} & =0
\end{aligned}
$$

- Derivatives relative to $x_{h}^{i^{\prime}}$ for $i, i^{\prime}=1, \ldots, I, k, h=1, \ldots, n$ :

$$
\begin{aligned}
\frac{\partial F_{(i-1) n+k}}{\partial x_{h}^{i}} & =-\beta \alpha 1_{h=k} u^{\prime \prime}(\delta+(P+y) x)(P+y)^{2} \\
F_{n L+k} \partial x_{h}^{i} & =1_{h=k} \eta^{i}
\end{aligned}
$$

- The Jabobian matrix $J_{F}$ has the following simple shape:

$$
\Delta=\left[\begin{array}{cccc}
I_{n}-\beta \varphi T & \psi I_{n} & \ldots & 0 \\
\vdots & 0 & \ddots & 0 \\
I_{n}-\beta \varphi T & 0 & \ldots & \psi I_{n} \\
0 & \eta^{1} I_{n} & \ldots & \eta^{I} I_{n}
\end{array}\right]
$$

where $I_{n}$ is the $n \times n$ identity matrix, $T=\left(\pi_{k, l}\right)_{1 \leq k, l \leq n}$ and scalars $0<\varphi<1$ and $\psi>0$ are defined as:

$$
\begin{aligned}
& \varphi=\beta\left(1-\alpha+\alpha u^{\prime}((P+y) x+\delta)+\alpha x u^{\prime \prime}((P+y) x+\delta)(P+y)\right) \\
& \psi=-\beta \alpha u^{\prime \prime}(\delta+(P+y) x)(P+y)^{2}
\end{aligned}
$$


- We prove that $J_{F}$ is invertible. Let $X=\left(X_{0}, X_{1}, \ldots, X_{I}\right) \in\left(R^{n}\right)^{I}$. If $X \in \operatorname{ker} \Delta$, then:

$$
\begin{aligned}
\forall i \in\{1, \ldots, I\},\left(I_{n}-\beta \varphi T\right) X_{0}+\psi X_{i} & =0_{n} \\
\sum_{i=1}^{I} \eta^{i} X_{i} & =0_{n}
\end{aligned}
$$

A linear combination of the first lines yields easily that $\left(I_{n}-\beta \varphi T\right)\left(\sum_{i=1}^{I} \eta^{i} X_{0}\right)=0_{n}$ and $X_{0}=0_{n}$ since $I_{n}-\beta \varphi T$ is invertible (the inverse is $\sum_{k=0}^{\infty} \beta^{k} \varphi^{k} T^{k}$ which exists since $0<\varphi<1$ ). It is then straightforward to deduce that $X_{i}=0_{n}$ for all $1 \leq i \leq I$.

The Jacobian of $F$ with respect to $Z$ is invertible at the point $\left(X^{*}, Z^{*}\right)$, which proves the equilibrium existence for parameters $X$ in the vicinity of $X^{*}$.

\section{J Proof of Proposition 10}

We use a proof strategy very similar to Section $\mathrm{D}$ We define $X=\left(y_{1}, \ldots, y_{n}, V, \alpha^{1}, \ldots, \alpha^{I}, w_{1}^{1}, \ldots, w_{n}^{1}, \ldots, w_{1}^{I}, \ldots, w_{n}^{I}, K_{1}, \ldots, K_{L}\right) \in$ $\left(\mathbb{R}^{+}\right)^{n+1+I+n I+L}$ the vector of parameters and $Z=\left(P_{1}, \ldots, P_{n}, x_{1}^{1}, \ldots, x_{n}^{1}, \ldots, x_{1}^{I}, x_{n}^{I}, Q_{1,1}, \ldots, Q_{n, 1}, \ldots, Q_{1, L}, \ldots, Q_{n, L}, s_{1,1}^{1}, \ldots, s_{n, 1}^{1}, \ldots, s_{1,1}^{I}\right.$, $\left.s_{1, L}^{1}, \ldots, s_{n, L}^{1}, \ldots, s_{n, 1}^{I}, \ldots, s_{1, L}^{I}, \ldots, s_{n, L}^{I}\right) \in\left(\mathbb{R}^{+}\right)^{n+n I+n L+n I L}$ the vector of endogenous variables.

We define the function $G:\left(\mathbb{R}^{+}\right)^{n+1+I+n I+L} \times\left(\mathbb{R}^{+}\right)^{n+n I+n L+n I L} \longrightarrow\left(\mathbb{R}^{+}\right)^{n+n I+n L+n I L}$ as follows $(i=$ $1, \ldots, I$ and $k=1, \ldots, n)$ :

$$
\begin{aligned}
G_{(i-1) n+k}(X, Z) & =\frac{P_{k}}{w_{k}^{i}}-\beta \sum_{j=1}^{n} \pi_{k, j}\left(\frac{1-\alpha^{i}}{w_{j}^{i}}+\alpha^{i} u^{\prime}\left(\delta+\left(P_{j}+y_{j}\right) x_{k}^{i}+\sum_{l=1}^{L}\left(P_{j}-K_{l}\right)^{+} s_{k, l}^{i}\right)\right)\left(P_{j}+y_{j}\right) \\
G_{n I+k}(X, Z) & =\sum_{i=1}^{I} \eta^{i} x_{k}^{i}-V \\
G_{(l-1) n I+(i-1) n+k}(X, Z) & =\frac{Q_{k, l}}{w_{k}^{i}}-\beta \sum_{j=1}^{n} \pi_{k, j}\left(\frac{1-\alpha^{i}}{w_{j}^{i}}+\alpha^{i} u^{\prime}\left(\delta+\left(P_{j}+y_{j}\right) x_{k}^{i}+\sum_{m=1}^{L}\left(P_{j}-K_{m}\right)^{+} s_{k, m}^{i}\right)\right)\left(P_{j}-K_{l}\right)^{+} \\
G_{n I L+n I+(l-1) n+k}(X, Z) & =\sum_{i=1}^{I} \eta^{i} s_{k, l}^{i}
\end{aligned}
$$

Symmetric equilibrium. We already now that for the set of parameters $X^{*}=\left(y_{1}, \ldots, y_{n}, V^{*}, \alpha, \ldots, \alpha, 1, \ldots, 1, K_{1}, \ldots, K_{L}\right)$ an equilibrium exists, such that $Z^{*}=\left(P_{1}, \ldots, P_{n}, x_{1}, \ldots, x_{n}, \ldots, x_{1}, x_{n}, Q_{1,1}, \ldots, Q_{n, 1}, \ldots, Q_{1, L}, \ldots, Q_{n, L}, 0, \ldots, 0\right)$. In this equilibrium, options are priced but not traded. Strikes and prices are such that: $P_{1}<K_{1}<P_{2}<K_{2}<$ $\ldots<P_{L}<K_{L}<P_{L+1}<\ldots<P_{n}$.

Implicit function theorem. We compute the Jacobian of $G$ in $\left(X^{*}, Z^{*}\right)$ and prove that is invertible. 
- We start with the derivatives relative to $P_{j}(j, k=1, \ldots, n$ and $i=1, \ldots I)$ :

$$
\begin{aligned}
\frac{\partial G_{(i-1) n+k}}{\partial P_{j}}\left(X^{\star}, Z^{\star}\right) & =1_{k=j}-\beta \pi_{k, j}\left(1-\alpha+\alpha u^{\prime}\left(\delta+\left(P_{j}+y_{j}\right) x_{k}\right)+\alpha x_{k} u^{\prime \prime}\left(\delta+\left(P_{j}+y_{j}\right) x_{k}\right)\left(P_{j}+y_{j}\right)\right) \\
\frac{\partial G_{n L+k}}{\partial P_{j}}\left(X^{\star}, Z^{\star}\right) & =0 \\
\frac{\partial G_{(l-1) n L+(i-1) n+k}}{\partial P_{j}}\left(X^{\star}, Z^{\star}\right) & =-\beta \pi_{k, j}\left(1-\alpha+\alpha u^{\prime}\left(\delta+\left(P_{j}+y_{j}\right) x_{k}\right)-\alpha x u^{\prime \prime}\left(\left(P_{j}+y_{j}\right) x_{k}+\delta\right)\left(P_{j}-K_{l}\right) 1_{j>l}\right) \\
\frac{\partial G_{n L M+k+n l}}{\partial P_{j}}\left(X^{\star}, Z^{\star}\right) & =0
\end{aligned}
$$

- We derive relative to $x_{h}^{i^{\prime}}$ :

$$
\begin{aligned}
\frac{G_{(i-1) n+k}}{\partial x_{h}^{i^{\prime}}}\left(X^{\star}, Z^{\star}\right) & =-1_{h=k} 1_{i^{\prime}=i} \beta \alpha \sum_{j=1}^{n} \pi_{k, j} u^{\prime \prime}\left(\delta+\left(P_{j}+y_{j}\right) x_{k}\right)\left(P_{j}+y_{j}\right)^{2} \\
\frac{G_{n L+k}}{\partial x_{h}^{i^{\prime}}}\left(X^{\star}, Z^{\star}\right) & =1_{h=k} \eta^{i^{\prime}} \\
\frac{G_{(l-1) n L+(i-1) n+k}}{\partial x_{h}^{i^{\prime}}}\left(X^{\star}, Z^{\star}\right) & =-1_{h=k} 1_{i^{\prime}=i} \beta \alpha \sum_{j=l+1}^{n} \pi_{k, j} u^{\prime \prime}\left(\delta+\left(P_{j}+y_{j}\right) x_{k}\right)\left(P_{j}+y_{j}\right)\left(P_{j}-K_{l}\right) \\
\frac{G_{n L M+k+n l}}{\partial x_{h}^{i^{\prime}}}\left(X^{\star}, Z^{\star}\right) & =0
\end{aligned}
$$

- The derivatives relative to $Q_{h, m}$ express as:

$$
\begin{aligned}
\frac{G_{(i-1) n+k}}{\partial Q_{h, m}}\left(X^{\star}, Z^{\star}\right) & =0 \\
\frac{G_{n L+k}}{\partial Q_{h, m}}\left(X^{\star}, Z^{\star}\right) & =0 \\
\frac{G_{(l-1) n L+(i-1) n+k}}{\partial Q_{h, m}}\left(X^{\star}, Z^{\star}\right) & =1_{h=k} 1_{m=l} \\
\frac{G_{n L M+k+n l}}{\partial Q_{h, m}}\left(X^{\star}, Z^{\star}\right) & =0
\end{aligned}
$$

- Finally, derivatives relative to $s_{h, m}^{i^{\prime}}$ :

$$
\begin{aligned}
\frac{G_{(i-1) n+k}}{\partial s_{h, m}^{i^{\prime}}}\left(X^{\star}, Z^{\star}\right) & =-1_{i^{\prime}=i} 1_{h=k} \alpha \beta \sum_{j=m+1}^{n} \pi_{k, j} u^{\prime \prime}\left(\delta+\left(P_{j}+y_{j}\right) x_{k}\right)\left(P_{j}+y_{j}\right)\left(P_{j}-K_{m}\right) \\
\frac{G_{n L+k}}{\partial s_{k, l^{\prime}}^{i}}\left(X^{\star}, Z^{\star}\right) & =0 \\
\frac{G_{(l-1) n L+(i-1) n+k}}{\partial s_{h, m}^{i^{\prime}}}\left(X^{\star}, Z^{\star}\right) & =-1_{i^{\prime}=i} 1_{h=k} \alpha \beta \sum_{j=1+\max (m, l)}^{n} \pi_{k, j} u^{\prime \prime}\left(\delta+\left(P_{j}+y_{j}\right) x_{k}\right)\left(P_{j}-K_{l}\right)\left(P_{j}-K_{m}\right) \\
\frac{G_{n L M+k+n l}}{\partial s_{h, m}^{i^{\prime}}}\left(X^{\star}, Z^{\star}\right) & =1_{k=h} 1_{m=l} \eta^{i^{\prime}}
\end{aligned}
$$


- We define now the following matrices $(l=1, \ldots, L)$ :

$$
\begin{aligned}
M_{y} & =\left(\pi_{k, l}\left(1-\alpha+\alpha u^{\prime}\left(\delta+\left(P_{j}+y_{j}\right) x_{k}\right)+\alpha x_{k} u^{\prime \prime}\left(\delta+\left(P_{j}+y_{j}\right) x_{k}\right)\left(P_{j}+y_{j}\right)\right)\right)_{1 \leq k, j \leq n} \\
M_{K_{l}} & =\left(\pi_{k, l}\left(1-\alpha+\alpha u^{\prime}\left(\delta+\left(P_{j}+y_{j}\right) x_{k}\right)+\alpha x_{k} u^{\prime \prime}\left(\delta+\left(P_{j}+y_{j}\right) x_{k}\right)\left(P_{j}-K_{l}\right)^{+}\right)\right)_{1 \leq k, j \leq n} \\
\Gamma & =\left(\pi_{k, j} u^{\prime \prime}\left(\delta+\left(P_{j}+y_{j}\right) x_{k}\right)\right)_{1 \leq k, j \leq n} \\
\Pi_{y} & =\left(P_{j}+y_{j}\right)_{1 \leq j \leq n} \in \mathbb{R}^{n} \\
\Pi_{K_{l}} & =\left(\left(P_{j}-K_{l}\right)^{+}\right)_{1 \leq j \leq n} \in \mathbb{R}^{n} \\
\Delta_{u v} & =-\alpha \beta \operatorname{diag}\left(\sum_{j=1}^{n} \pi_{k, j} u^{\prime \prime}\left(\delta+\left(P_{j}+y_{j}\right) x_{k}\right)\left(\Pi_{u}\right)_{j}\left(\Pi_{v}\right)_{j}\right)_{k=1, \ldots, n} \quad \text { with } u, v=y, K_{l} \\
I_{n} & =n \times n \text { identity matrix } \\
J_{n} & =(1) \in \mathbb{R}^{n} \times \mathbb{R}^{n} \\
E_{k, l} & =\left(e_{i, j}^{k, l}\right)_{1 \leq i, j \leq n} \text { with } e_{i, j}^{k, l}=1_{k=i} 1_{l=j}
\end{aligned}
$$

- The Jacobian $J_{G}$ expresses as follows:

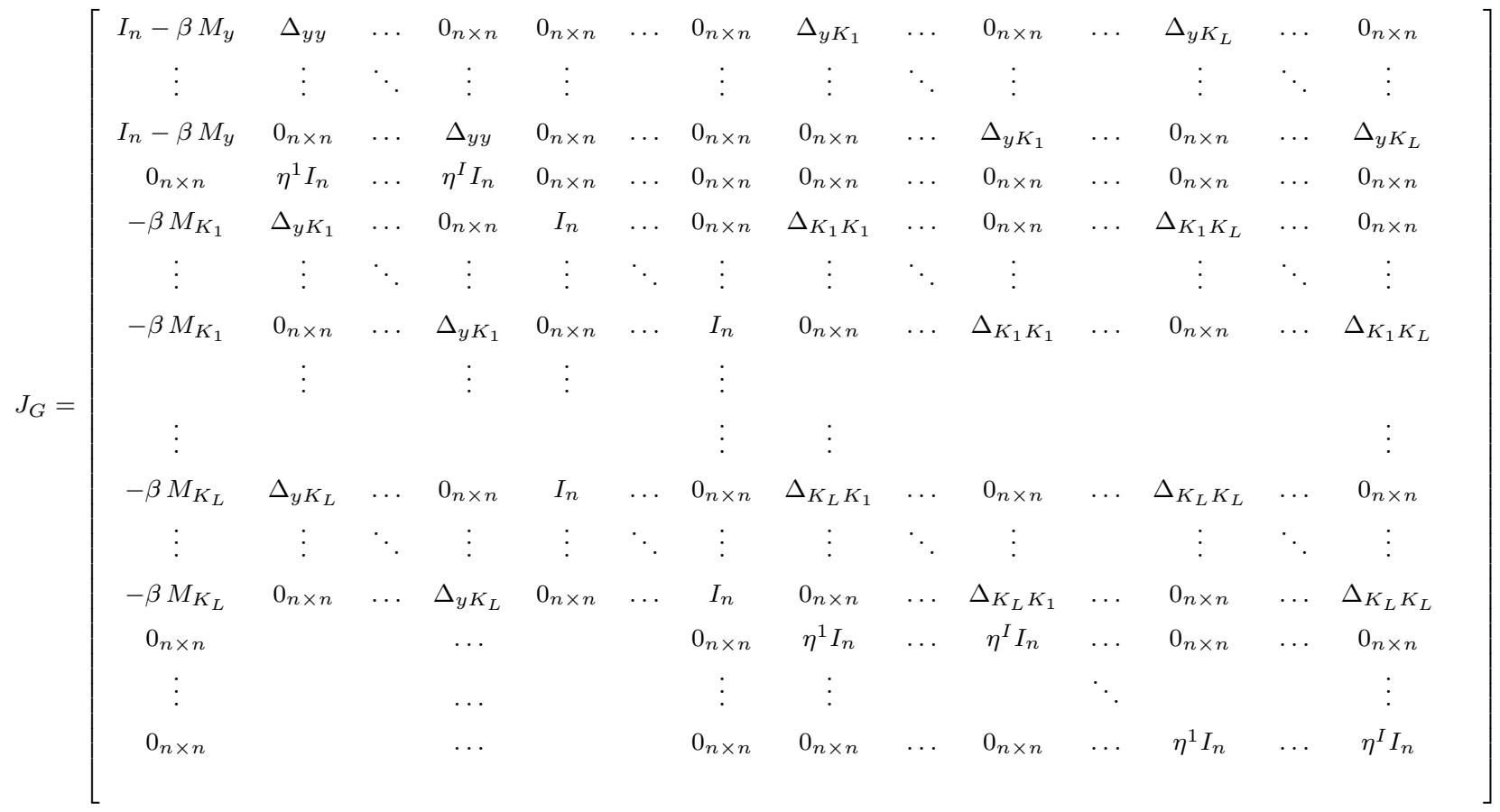

- Let $X=\left(X_{0},\left(X_{1}^{i}\right)_{i=1, \ldots, I},\left(X_{2}^{l}\right)_{l=1, \ldots, L},\left(X_{3}^{i, l}\right)_{i=1, \ldots, I ; l=1, \ldots, L}\right) \in \mathbb{R}^{n} \times\left(\mathbb{R}^{n}\right)^{I} \times\left(\mathbb{R}^{n}\right)^{L} \times\left(\mathbb{R}^{n}\right)^{I \times L}$. Suppose 
$X \in \operatorname{ker}\left(J_{G}\right)$. We thus have the following system:

$$
\begin{aligned}
\forall i \in\{1, \ldots, I\}, \quad\left(I_{n}-\beta M_{y}\right) X_{0}+\Delta_{y y} X_{1}^{i}+\sum_{m=1}^{L} \Delta_{y K_{m}} X_{3}^{i, m} & =0_{n} \\
\sum_{i=1}^{I} \eta^{i} X_{1}^{i} & =0_{n} \\
\forall i \in\{1, \ldots, I\}, \forall l \in\{1, \ldots, L\}, \quad-\beta M_{K_{l}} X_{0}+\Delta_{y K_{l}} X_{1}^{i}+X_{2}^{l}+\sum_{m=1}^{L} \Delta_{K_{l} K_{m}} X_{3}^{i, m} & =0_{n} \\
\forall l \in\{1, \ldots, L\}, \quad \sum_{i=1}^{I} \eta^{i} X_{3}^{i, l} & =0_{n}
\end{aligned}
$$

Using a proper linear combination of 46 together with 47 and 49 , we deduce $\left(I_{n}-\beta M_{y}\right)\left(\sum_{i=1}^{I} \eta^{i}\right) X_{0}=$ $0_{n}$. Since $\sum_{i=1}^{I} \eta^{i}>0$ and $I_{n}-\beta M_{y}$ invertible, we conclude $X_{0}=0_{n}$. An analogous manipulation yields $X_{2}^{l}=0_{n}$ for $l=1, \ldots, L$. The previous system simplifies into:

$$
\begin{array}{r}
\forall i \in\{1, \ldots, I\}, \Delta_{y y} X_{1}^{i}+\sum_{m=1}^{L} \Delta_{y K_{m}} X_{3}^{i, m}=0_{n} \\
\forall i \in\{1, \ldots, I\}, \forall l \in\{1, \ldots, L\}, \quad \Delta_{y K_{l}} X_{1}^{i}+\sum_{m=1}^{L} \Delta_{K_{l} K_{m}} X_{3}^{i, m}=0_{n} \\
\forall l \in\{1, \ldots, L\}, \quad \sum_{i=1}^{I} \eta^{i} X_{3}^{i, l}=\sum_{i=1}^{I} \eta^{i} X_{1}^{i}=0_{n}
\end{array}
$$

We only need to prove that $X_{3}^{i, m}=0_{n}$. Since $\Delta_{y y}$ is invertible, we deduce that:

$\forall i \in\{1, \ldots, I\}, \forall l \in\{1, \ldots, L\}, \quad \sum_{m=1}^{L}\left(\Delta_{K_{l} K_{m}}-\Delta_{y K_{l}} \Delta_{y y}^{-1} \Delta_{y K_{m}}\right) X_{3}^{i, m}=0_{n}$

$\forall i \in\{1, \ldots, I\}, \forall l \in\{1, \ldots, L\}, \forall k \in\{1, \ldots, n\}$

$$
\sum_{m=1}^{L}\left(\sum_{j=1}^{n} \pi_{k, j} u_{k, j}^{\prime \prime}\left(P_{j}-K_{l}\right)^{+}\left(P_{j}-K_{m}\right)^{+}-\frac{\left(\sum_{j=1}^{n} \pi_{k, j} u_{k, j}^{\prime \prime}\left(P_{j}+y_{j}\right)\left(P_{j}-K_{m}\right)^{+}\right)\left(\sum_{j=1}^{n} \pi_{k, j} u_{k, j}^{\prime \prime}\left(P_{j}-K_{l}\right)^{+}\left(P_{j}+y_{j}\right)\right)}{\sum_{j=1}^{n} \pi_{k, j} u_{k, j}^{\prime \prime}\left(P_{j}+y_{j}\right)^{2}}\right) X_{3, k}^{i, m}=0
$$

Noting $\Lambda_{k}=\operatorname{diag}\left(-\pi_{k, j} u_{k, j}^{\prime \prime}\right)_{j=1, \ldots, n}$, we define the bilinear form $(\cdot \mid \cdot)_{k}$ as follows:

$$
\forall U, V \in\left(\mathbb{R}^{n}\right)^{2},(U \mid V)_{k}=U^{\top} \Lambda_{k} V=-\sum_{j=1}^{n} \pi_{k, j} u_{k, j}^{\prime \prime} U_{j} V_{j}
$$

It is straightforward to verify that $(\cdot \mid \cdot)_{k}$ is a scalar product. Using this scalar product, we obtain after some simple manipulations:

$$
\forall l \in\{1, \ldots, L\},\left(\left(\Pi_{y} \mid \Pi_{y}\right)_{k}\left(\Pi_{K_{l}} \mid \sum_{m=1}^{L} \Pi_{K_{m}}\right)_{k}-\left(\Pi_{K_{l}} \mid \Pi_{y}\right)_{k}\left(\Pi_{y} \mid \sum_{m=1}^{L} \Pi_{K_{m}}\right)_{k}\right) X_{3, k}^{i, m}=0
$$

Summing over $l$ yields:

$$
\forall l \in\{1, \ldots, L\},\left(\left(\Pi_{y} \mid \Pi_{y}\right)_{k}\left(\sum_{m=1}^{L} \Pi_{K_{m}} \mid \sum_{m=1}^{L} \Pi_{K_{m}}\right)_{k}-\left(\Pi_{y} \mid \sum_{m=1}^{L} \Pi_{K_{m}}\right)_{k}^{2}\right) X_{3, k}^{i, m}=0
$$


Since $\sum_{m=1}^{L} \Pi_{K_{m}}$ and $\Pi_{y}$ are not collinear (by construction of options, which do not replicate the asset), the Cauchy-Schwarz inequality implies that:

$$
\left(\Pi_{y} \mid \Pi_{y}\right)_{k}\left(\sum_{m=1}^{L} \Pi_{K_{m}} \mid \sum_{m=1}^{L} \Pi_{K_{m}}\right)_{k}-\left(\Pi_{y} \mid \sum_{m=1}^{L} \Pi_{K_{m}}\right)_{k}^{2}>0
$$

We conclude that for all $1 \leq i \leq L, 1 \leq m \leq L$, for all $1 \leq k \leq n$, we have $X_{3, k}^{i, m}=0$. In consequence, $X \in \operatorname{ker}\left(J_{G}\right)$ iif $X=0$. The Jacobian $J_{G}$ is therefore invertible in $\left(X^{*}, Z^{*}\right)$, which concludes the proof: The equilibrium exists in the vicinity of $\left(X^{*}, Z^{*}\right)$. 